\title{
EXPLORING THE SUSTAINING FACTORS THAT MOTIVATE NURSES TO WORK IN THE RURAL AREAS OF PAPUA NEW GUINEA
}

by

Priscilla Poga

A thesis submitted to Victoria University of Wellington in partial fulfilment of the requirements for the degree of Master of Health Care

Victoria University of Wellington

2019 


\begin{abstract}
Papua New Guinea is among other developing countries that are faced with a critical shortage in human resources in health, specifically nurses, and rural areas are the most affected. Initially, and perhaps unsurprisingly, there were only two relevant studies that directly related to Papua New Guinea in this area based on the literature search. The first of these two studies was focused on nurses and the social aspect of rural motivation compared to the other which was focused on rural health professionals in general. Nevertheless, other research studies were eventually found from other developing countries such as certain Pacific, African and Latin American countries that served to assist in focusing the research on the chosen topic.
\end{abstract}

This descriptive-exploratory study set out to explore the sustaining factors that influenced existing nurses to remain (or otherwise) in their work in Papua New Guinea's rural areas. As such, the study involves 10 rural nurses with over two years of rural work experience in two different organisations; government and church. The interviews were semi-structured and were designed to explore the motivating factors for rural nurses and how any challenges, or demotivating factors, were overcome. The interviews were conducted in the common spoken language Tok Pisin which was translated into English, transcribed and analysed thematically.

Overall the study found that rural nurses are disadvantaged because they struggle with limited resources to deliver effective health care, and they also face several personal challenges which are often overlooked. The main findings are categorised under two major themes, 1) safety and 2) socioeconomic, and each are explored by further exploration of the themes and sub-themes that are evident in both. The implications of this study are examined, including recommendations, to develop policies that are designed to address the ongoing needs of rural nurses in Papua New Guinea.

Keywords: Papua New Guinea, motivation, rural, nurses 


\section{Acknowledgements}

I would like to express my appreciation for those people who have contributed in different ways to the thesis.

First and foremost, thank you to the ten participants of the study. It was very challenging to set aside some of your valuable time for the interviews especially in the rural health facilities where you are assigned to do many tasks within a very busy working day. Your commitment to slot in the interview within your busy schedules is much valued.

To Dr Martin Woods, my thesis supervisor, for your guidance and support throughout the journey. Your words of encouragement have motivated me this far.

To Laura Margaret Kamau from the student learning centre who often exceeded the required hours to provide extra support and guidance. Thank you very much for making time available for me.

To Dr Katherine Nelson, for always making time available and providing assistance. I really appreciate your help.

To the hospital managers of the three hospitals in which I have conducted my study. Thank you very much for your willingness and organising the participants. You all have made the interviews possible.

A special acknowledgement to my family in Papua New Guinea. Foremost to my late father Paul Poga for always believing in me and never giving up on supporting me. Your words "Success is possible through perseverance and determination" have motivated me to face challenges with positivity. I wish you were here to read this but it's not my place to question, only God knows why. You will forever be remembered in my heart dad. Rest in love till we meet again on that golden shore. 
To my fiancé Martin Mathew, mother Diniso Poga and siblings Nowake, Jennitha, Paulus, Christopher, and Alphie. Thank you all for your support and prayers.

To people who have accompanied me in my travels to rural locations for data collection, Unako, Johnathan, Joeboy, Mavire, and Tei. Thank you very much for your assistance in making this data collection possible.

To the Ministry of Foreign Affairs and Trade (MFAT) for offering me a scholarship to undertake this course. Thank you very much for the opportunity. 


\section{Table of Contents}

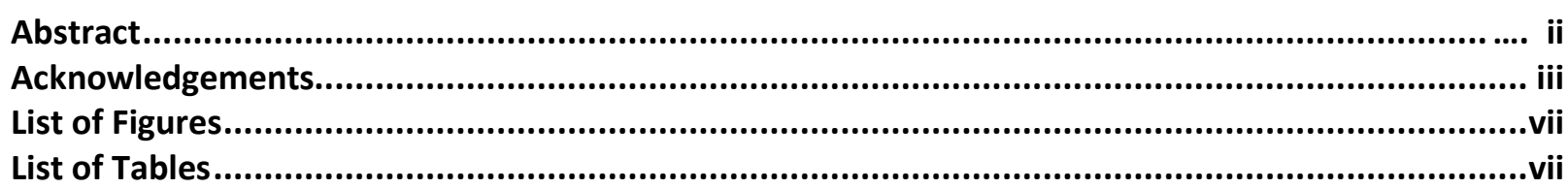

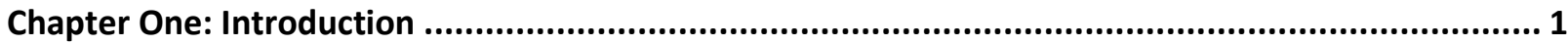

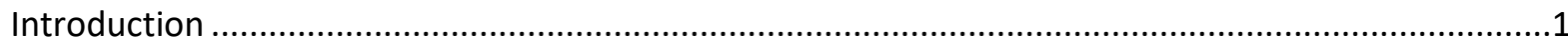

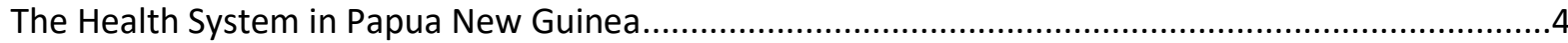

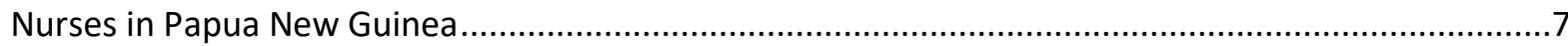

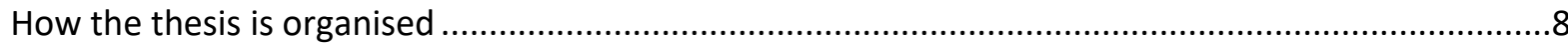

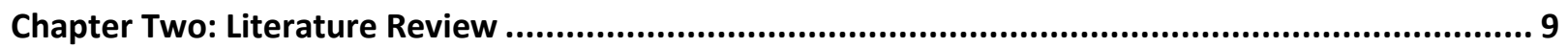

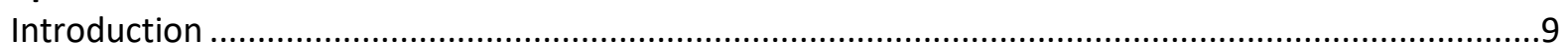

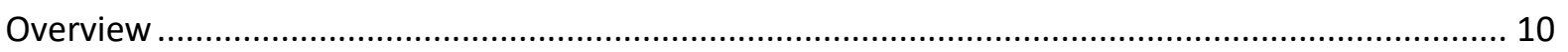

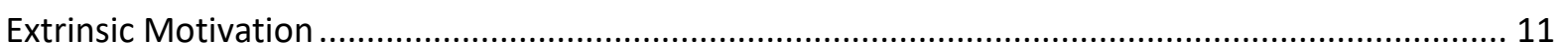

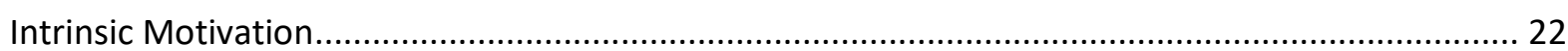

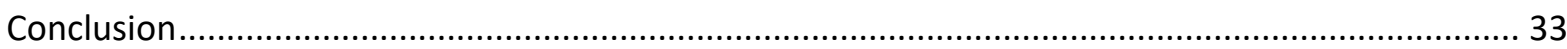

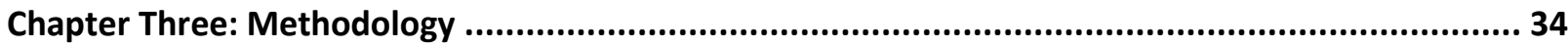

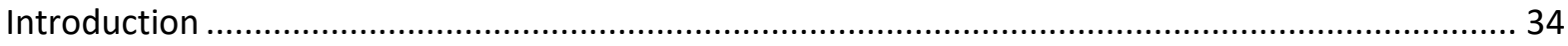

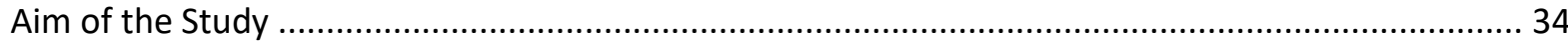

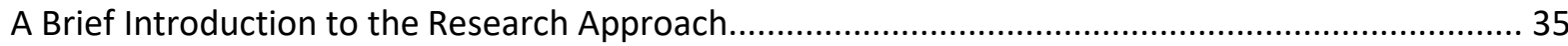

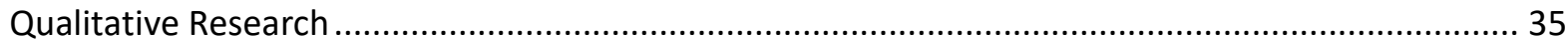

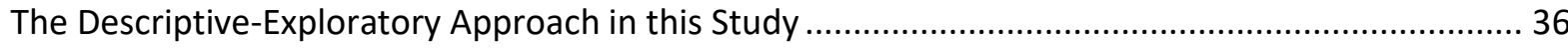

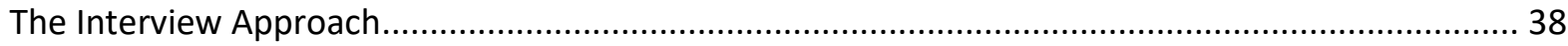

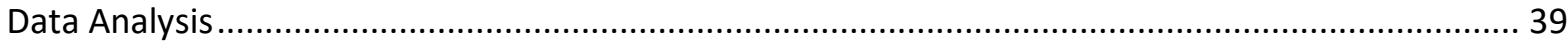

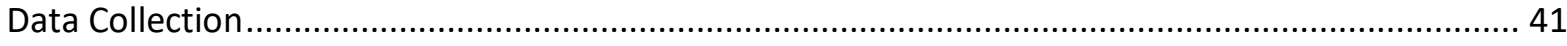

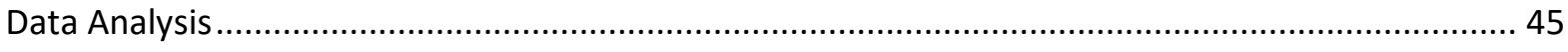

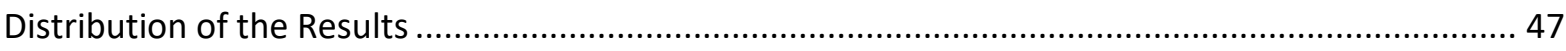

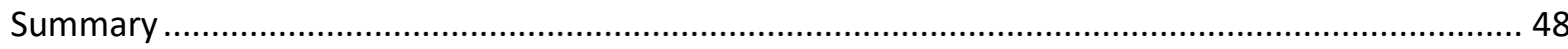

Chapter Four: Findings

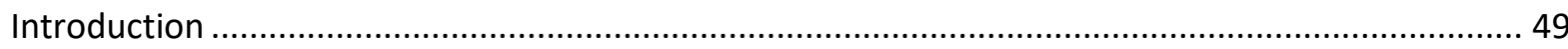

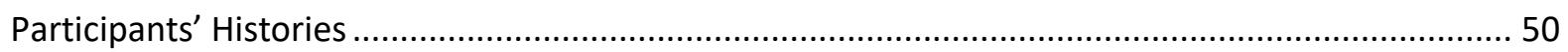


An Overview of Nursing Responsibilities in the Rural Areas of Papua New Guinea.......................... 51

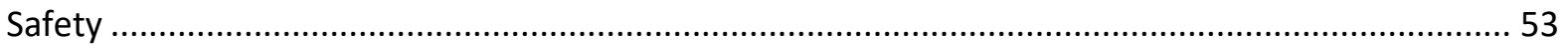

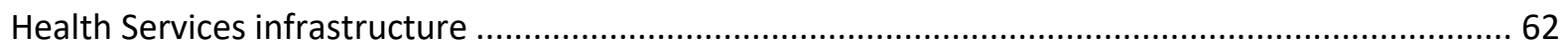

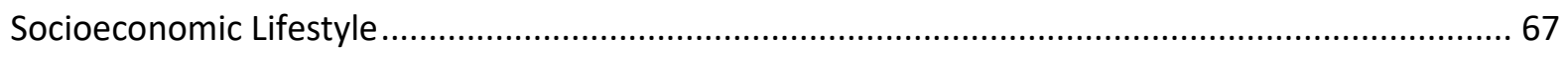

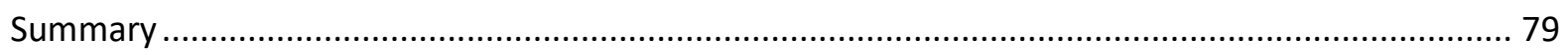

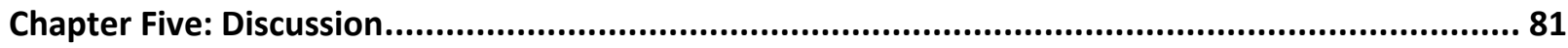

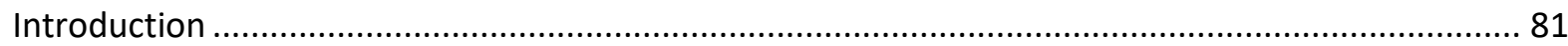

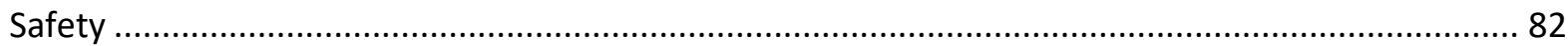

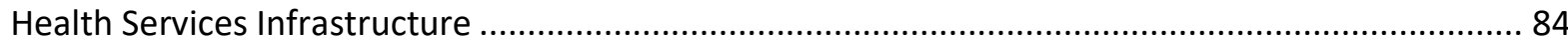

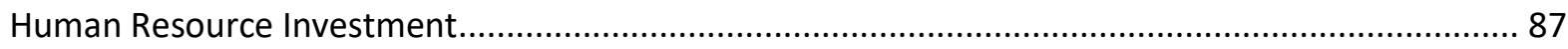

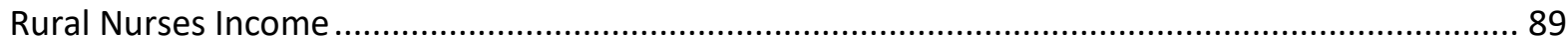

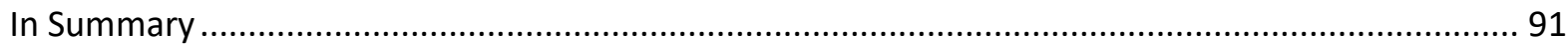

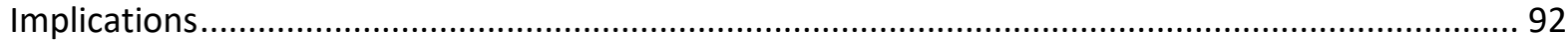

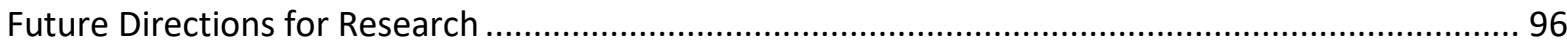

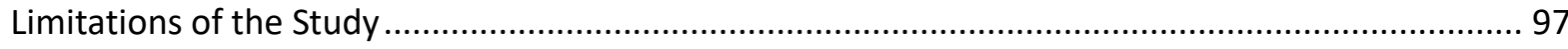

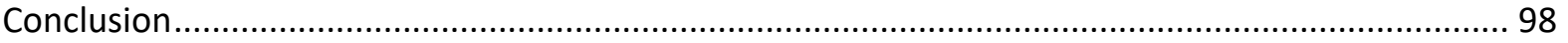

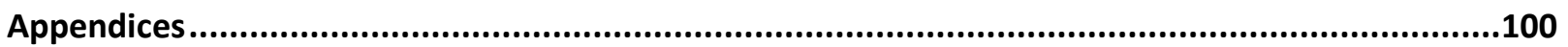

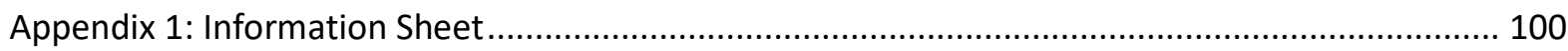

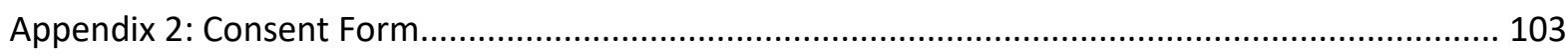

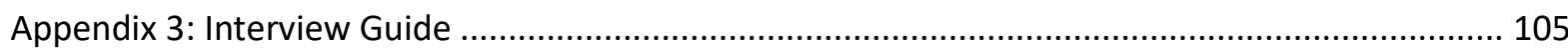

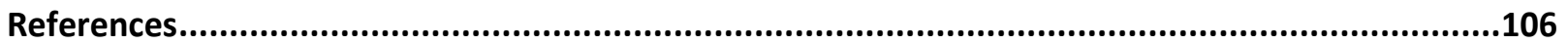




\section{List of Figures}

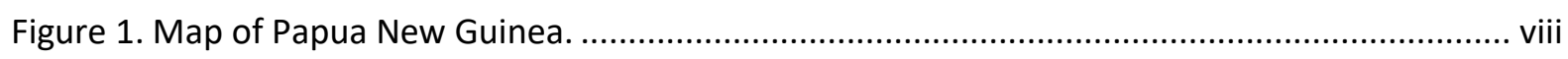

Figure 2. Political arrangement in Papua New Guinea. .................................................................. viii

Figure 3. Man carrying his sick wife through the tropical rain forest to the health facility................. 2

Figure 4. Health centre in rural Papua New Guinea. ................................................................... 2

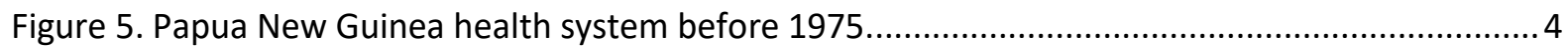

Figure 6. Papua New Guinea health system after 1975.............................................................. 5

Figure 7. Initial thematic map showing five main themes ........................................................... 40

Figure 8. Final thematic map, showing final two themes............................................................... 41

Figure 9. Final themes for nurses' motivation in rural areas of Papua New Guinea...........................4 47

Figure 10. Motivational factors for rural nurses in Papua New Guinea. .......................................... 49

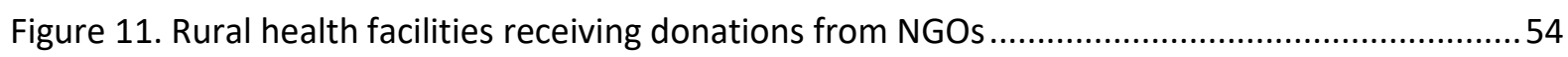

Figure 12 Rural hospital using tank water for health care.......................................................... 59

Figure 13. Poor hospital building in one of the Papua New Guinea rural location. ...........................63

Figure 14. Ambulance transporting sick people from remote areas to rural hospital stuck in mud....65

Figure 15. Nurse incorporating religious beliefs into health care. ................................................ 76

Figure 16. Traditional beliefs and approach of healing. ............................................................. 78

\section{List of Tables}

Table 1 - Initial phase of thematic codes for rural nurses' motivation in PNG.....................................46

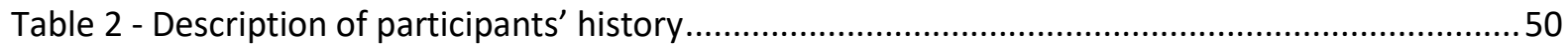




\section{Map and Political Arrangement of Papua New Guinea}

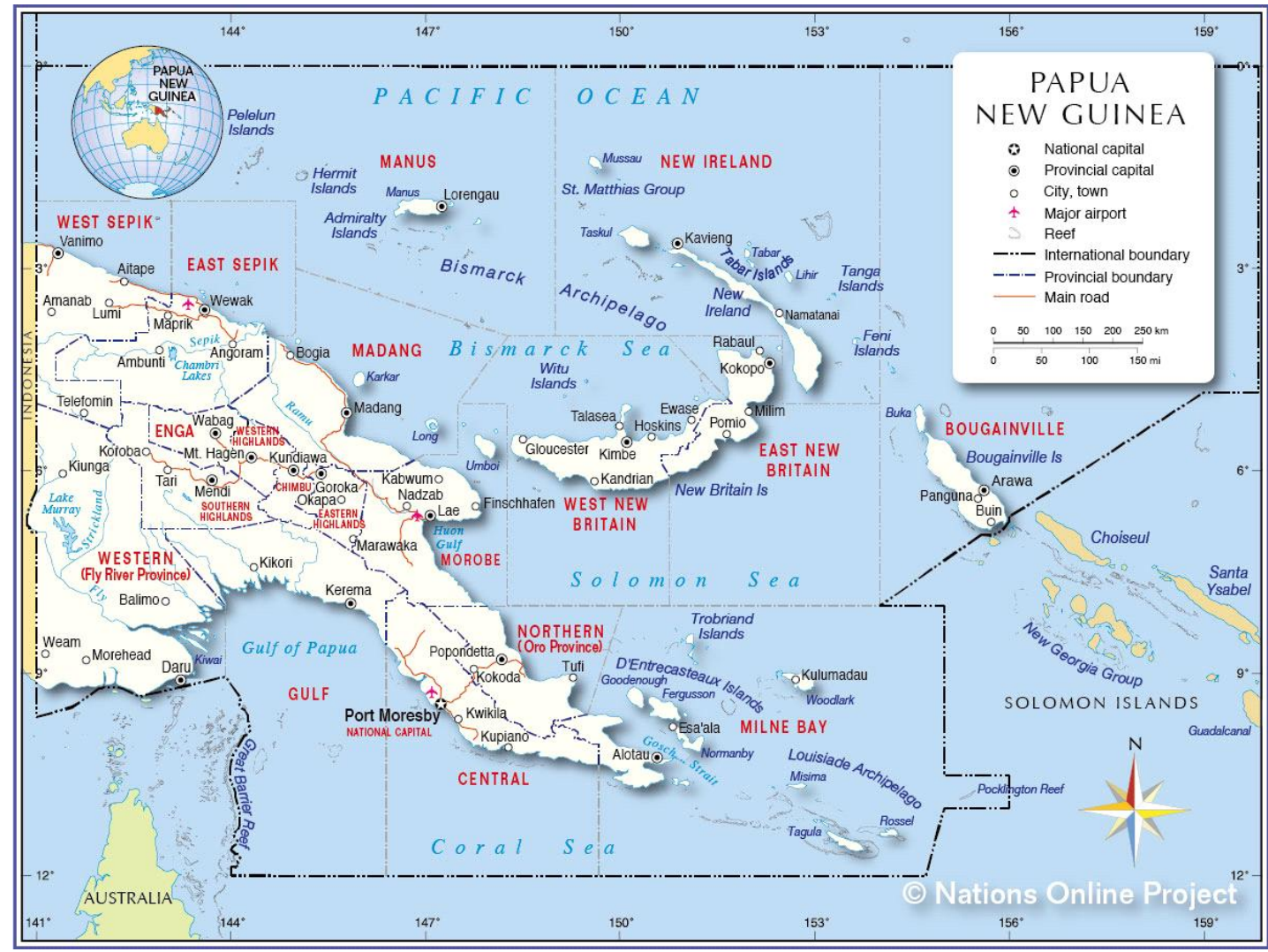

Figure 1. Map of Papua New Guinea.

Papua New Guinea is one of the largest Islands in the Pacific, it lies entirely in the tropics and is $160 \mathrm{~km}$ north of Australia. The country has four official languages, English, Sign Language, Tok Pisin, and Hiri Motu with 22 Provinces, 87 districts, and 300 Local Level Governments.

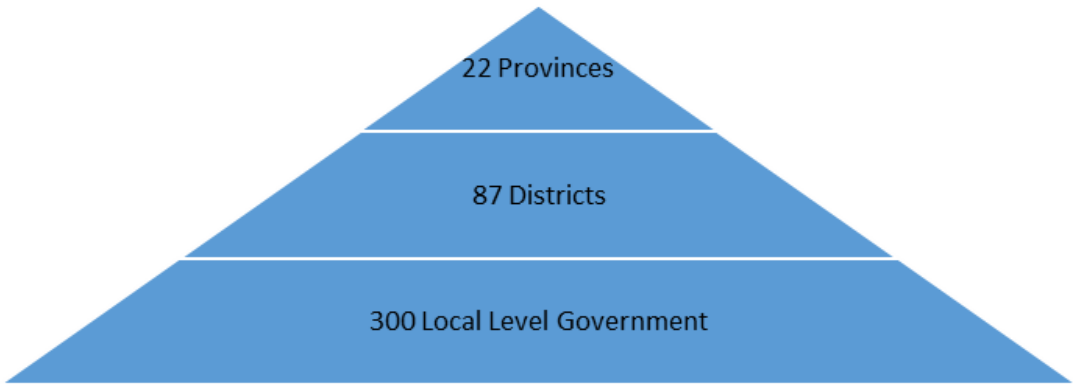

Figure 2. Political arrangement in Papua New Guinea 


\section{Chapter One: Introduction}

\section{Introduction}

Papua New Guinea (PNG) is one of the most diverse countries in the Pacific Island nations; it is home to more than 7.5 million people with over $82 \%$ of the total population living in rural areas where they survive by subsistence farming (The World Bank, 2017). The country has diverse cultures and traditions with four official languages, more than 800 native languages, and over 1,000 dialects that spread across four main regions of the country (Levy, 2005). It is known as the world's least explored nation with geographical and cultural varieties of as yet uncategorised flora and fauna. In addition, this nation is identified as one of the world's richest countries in terms of minerals and natural resources (Institute of National Affairs, 2007). As is the case in any other developing country PNG is faced with various issues with perhaps the main challenge being the declining health status of its population.

The poor health status of Papua New Guineans is an obvious fact that is revealed by its mortality rates. PNG has frequently been reported by the World Health Organization (WHO) as having the worst health status, including both the highest mortality and morbidity rates, in the Pacific Region (The National, 2015). Its maternal mortality rate is also one of the highest in the world (Department of National Planning and Monitoring, 2010b). The most affected population is the rural one with a much higher rate of maternal deaths reported annually than urban dwellers (Oxford Business Group, 2013). These statistics imply that the healthcare of the rural areas is in a dire state compared to urban populations. The main cause of this problem is the distance from suitable health facilities; it may take days to walk to reach the nearest health centre (Figure 3), and even there, facilities are often only very basic (Figure 4). In such centres, there are other causes that worsen the situation, the recent report from the media pointed out that the lack of pharmaceutical drugs and a significant shortage of healthcare personnel are known to be the leading causes of health related issues in PNG (Radio New Zealand, 2018). This means that besides other critical challenges, lack of health service providers is currently one of the most significant issues in rural health. 


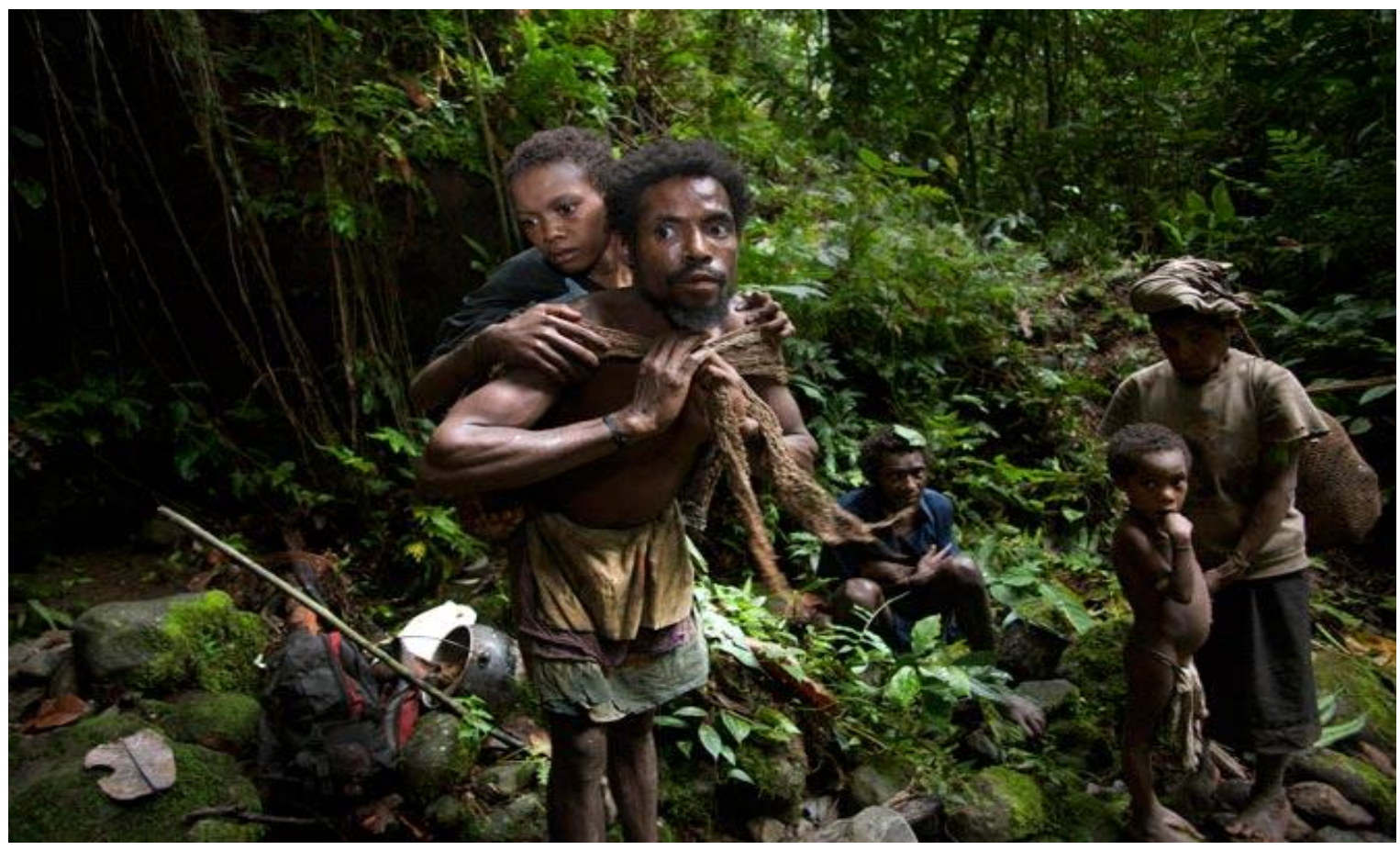

Figure 3. Man carrying his sick wife through the tropical rain forest to the health facility.

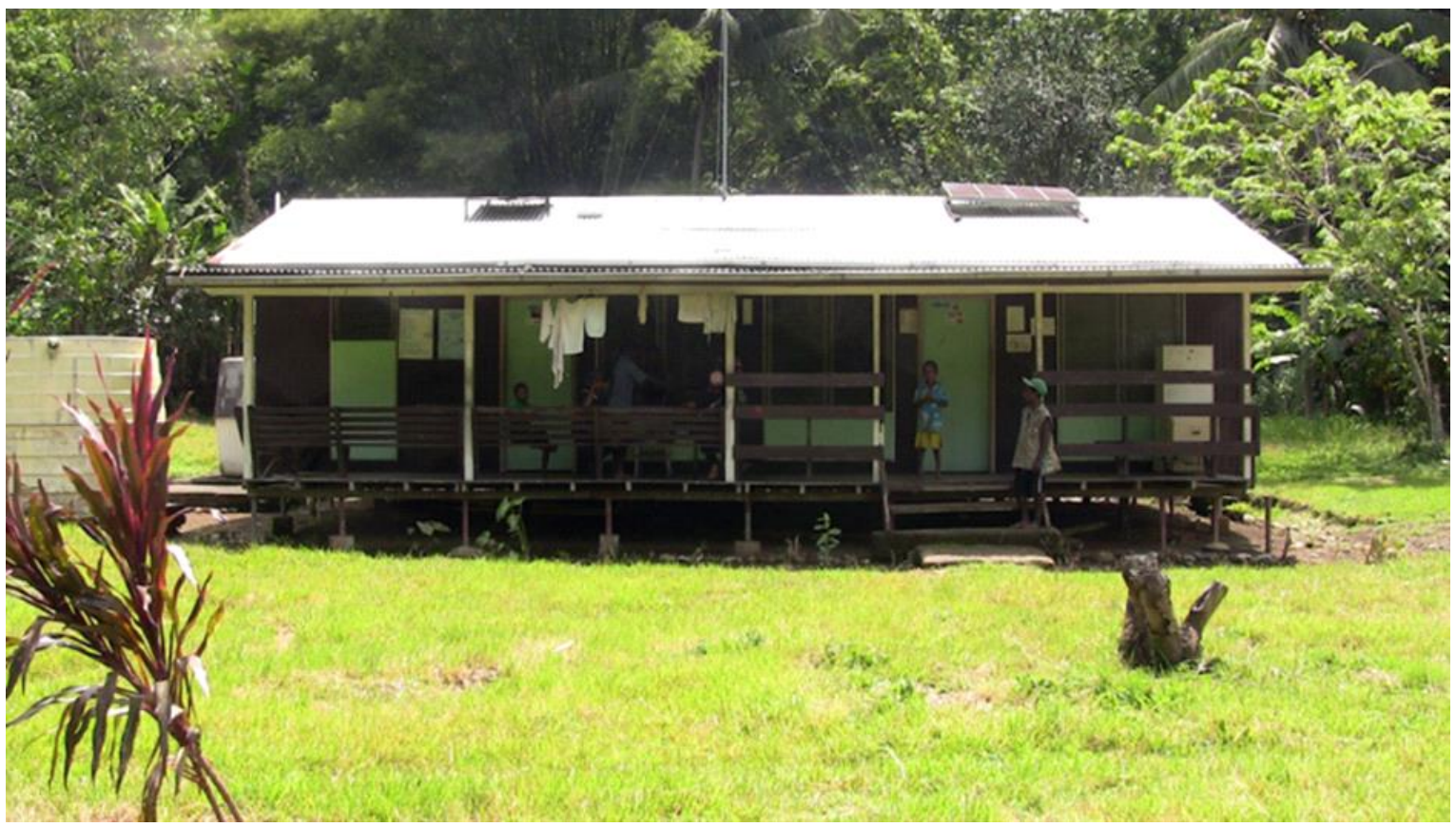

Figure 4. A typical Health Centre in rural Papua New Guinea.

Rural locations in PNG are unappealing to most nurses, the majority of them preferring urban locations. However, there are some nurses who are concerned about their communities and 
prefer working in rural health centres that are located near their community despite being demotivated by various issues. Rural health centres as shown in Figure 4 are smaller than the rural hospitals and are located in the remotest parts of the district; they only provide basic health care such as drug administration and injection services. However, due to the longdistance to travel to reach the nearest rural hospital, rural nurses in health centres often assist with emergencies, many of which are labour cases. Despite lacking most of the required equipment for these procedures, the nurses often improvise to save a patient's life when possible to patients that require assistance beyond the nurses' knowledge or the hospital stock of suitable medical supplies are referred to rural hospitals.

Working in rural and remote areas where health care is in constant demand often forces nurses to work during their off days to serve their communities, either in the hospitals, at their homes, or within their communities. This type of practice is called the wantok system in PNG (in Tok Pisin, wantok means "one talk"). This ancient system of community care implies that tribal members are socially responsible for each other's welfare. The rural health facilities in regard to its population and health status need nurses who can serve their own community based on this wantok system approach but so far very few accept rural offers with the majority turning down these offers.

As such, this thesis is centred on rural health workers, with the main focus being on the existing rural nursing population. It is framed to identify the factors that have impacted the lives of individual nurses practising in rural health facilities in PNG. In particular, it is based on the notion that there are factors that either encourage or discourage nurses to remain in or leave rural health positions. It is anticipated that the factors identified by this study will assist the management of the rural health facilities to perform accordingly to meet the expectation of the rural health workers. Also, it will further strengthen the findings of other previous studies on similar issues and will stimulate the Provincial Health Authority (PHA) and District Health Authority (DHA) who are responsible for policy implementation to be effective in implementing some developed policies that are related to addressing these issues. Furthermore, it will assist 
the National Department of Health $(\mathrm{NDoH})$ to create some specific policies to address the needs of the rural nurses in order to motivate and sustain more workers.

Overall, it is hoped that this thesis will help the authorities in charge of the rural health facilities in PNG to improve some of their functions in relation to providing and maintaining adequate nursing personnel in the rural areas of PNG. Moreover, it will highlight for the health sector the main reasons behind the gradual decline of rural nurses so that they are able to construct effective plans and policies to address the underlying issues that should motivate and retain nurses. To assist in a fuller understanding of the relevance of the study, a description of rural health functions and their authorities is provided.

\section{The Health System in Papua New Guinea}

Figures 5 and 6 illustrate the different health systems employed in the PNG health sector preand post-independence. Figure 5 shows the centralised system, a system that was used before independence in 1975 while Figure 6 shows the decentralised system which was introduced after independence and remains the current administrative structure.

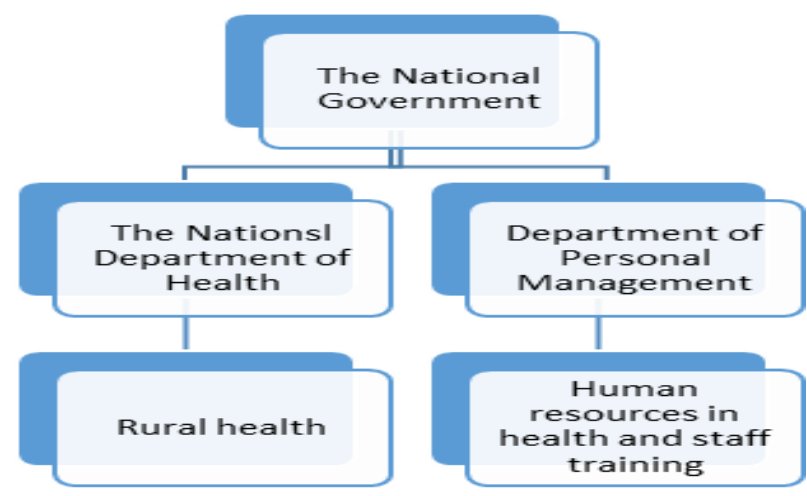

Figure 5. Papua New Guinea health system before 1975. 


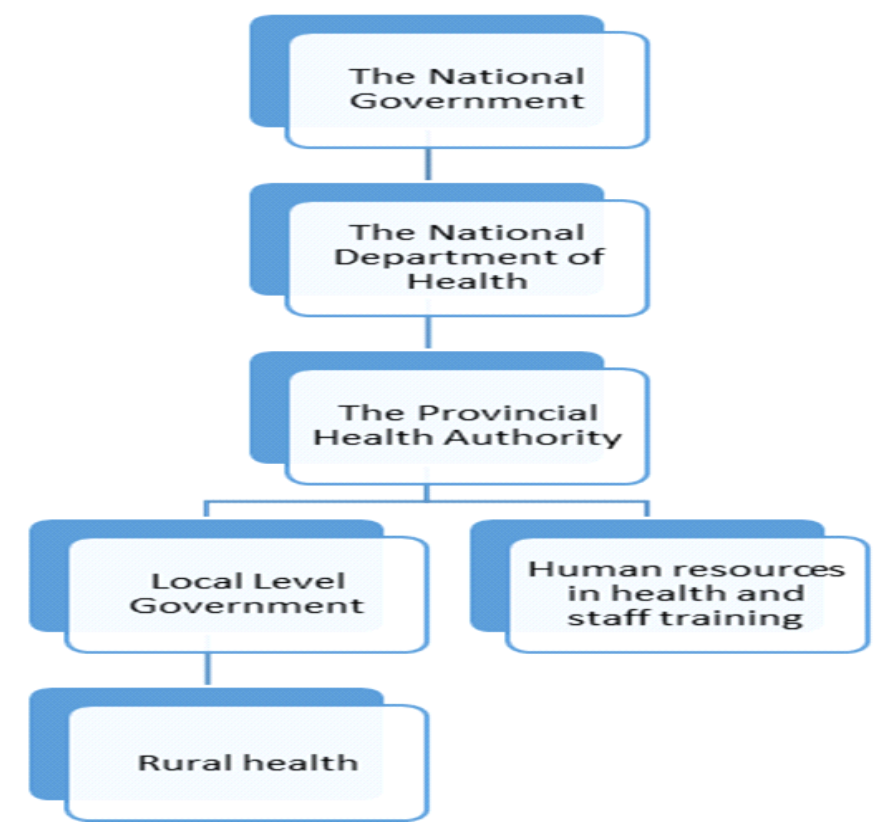

Figure 6. Papua New Guinea health system after 1975.

In pre-independence days, the health system in PNG was heavily centralised. In this system the $\mathrm{NDoH}$ oversaw the nation's health issues and provided service directly wherever they were needed. This meant that rural health communicated directly with NDoH which was responsible for meeting all of the health needs of the population. Alongside the NDoH, the Department of Personal Management (DPM) was intensively involved in the human resources of the health system. This department monitored the movement of health professionals and oversaw their training and education. Through this system, there were more health facilities in rural locations with an adequate workforce to meet the demand and communication between facilities was strong with less health issues as a result of the centralised structure.

However, immediately after independence in 1977 decentralisation was introduced complete with a different structure (Figure 6). In the decentralised system, the NDoH restructured and allocated responsibilities to different levels. In this restructuring, the country was divided into 22 provinces and provincial governments were introduced. During this process, the substantial powers held at national levels were transferred to provincial governments. Furthermore, the DPM which was once in-charge of public workers monitoring and training withdrew from its responsibilities and transferred these roles to the respective departments. Human resources in 
health and the responsibility to govern its own workers was one of these areas that was handed over to the NDoH. This role was further redirected to the PHA by NDoH. However, in the process of transferring the role, the function was not defined clearly to NDoH by DPM (Kolehmainen-Aitken, 1992). Subsequently, without being fully aware of the role PHA had accepted, less was done to suppress the growing decline in health workers, such as nurses, in rural areas over the next several years.

It has been argued that the introduction of the new health system in 1977 was initial cause of health workers refusing rural offers of work (Kolehmainen-Aitken, 1992). This was acknowledged by $\mathrm{NDoH}$ although surprisingly there was further decentralisation in 1995 to approximately 300 Local Level Government (LLG) organisations (Thomason \& Kase, 2009). The main objective for this massive decentralisation was for the LLG to deliver services effectively in areas particularly the rural locations where the national government might lack insight (Barcson, 2015). This implies that further decentralisation was meant for the LLG to actively engage all the rural health facilities in their specific zones and identify the reasons why nurses were declining in rural areas. Thus, it was argued, the strategies implemented would best suit their localities and the nurses' intake to such locations would improve as a result. Yet, with the intention to improve health workforce, the revised system actually confused the role between PHA and LLG. Thus, potential and actual health personnel were greatly affected by inconsistencies and inefficiencies in this system which emerged to the attention of NDoH.

These shifts of responsibilities without any necessary safeguards and checks resulted in the rural facilities lacking workers due to a lack of adequate social investment. One of the unforeseen effects of poor implementation of policy was the health workforce development; that is, a result of removing the DPM was a deterioration in human resources (KolehmainenAitken, 1992). Furthermore, it is noted that decentralisation has had direct impacts on the decline of the rural health workers, and specifically nurses. This is a major reason why this research was framed specifically to examine and outline the sustaining factors (and also by 
default, the non-sustaining factors) that motivated nurses to work in rural areas in the decentralised PNG health system.

\section{Nurses in Papua New Guinea}

Nurses make up more than $80 \%$ of the total population of health workers in PNG (Henderson \& Tulloch, 2008). Yet although nurses comprise a large proportion of the health professionals in that nation, there is an unequal distribution of them in favour of urban locations. The statistics shows that PNG has an estimate of 11,000 nurses, 5,600 of these health workers serve in rural locations while the rest are located in urban health facilities. (NDoH, 2012). However, this means that 5,400 serve less than $15 \%$ of the population, whilst 5,600 nurses serve over $85 \%$ of the total population. Hence, when comparing the population to the nurses, there is a clear imbalance in the distribution of nurses and 5,600 nurses is insufficient to meet the health demands of the increasing rural population. This issue is highly likely to worsen if PHAs and DHAs are inactive in policy implementation.

Apart from poor implementation of human resource policies at the two lower levels, another contributing factor is the lack of research to stimulate the policy. For instance, the extensive online literature searches that were performed in preparation for this study identified only two relevant articles of which one was on PNG rural health professionals in general while the second study was centred on the social aspects of rural nurses (Jayasuriya, Whittaker, Halim, \& Matineau, 2012; Razee, Whittaker, Jayasuriya, Yap, \& Bretnall, 2012). There were several other studies in regard to rural health in PNG, however this study selected the two research articles which were the most relevant to the thesis topic. Only two relevant studies specific to rural nurses implies that the developed polices will most likely remain inactive because of insufficient and limited evidence. This research therefore acknowledges the work of other health professionals in rural areas but concentrates on rural nurses as the focus of study in an attempt to encourage and support changes within that systetice. Therein, this research seeks to understand the shortage of nurses in these areas and argues that this issue is one of the major problems affecting rural health in PNG. 


\section{How the thesis is organised}

\section{Chapter two: Literature review}

This chapter discusses past research and related literature. It is based on journals and articles obtained from using search engines. However, it seems that PNG lacks studies on the specific topic of rural nurses because the extensive web search showed only two relevant studies. Hence, the study was supplemented by literature from other Pacific Island countries including other developing and developed countries. This chapter evaluates and integrates previous research and further explains how the research has been integrated into the current research program. The literature is explicitly explained from all sides of the argument to avoid bias and the chapter also highlights compatibilities and incompatibilities.

\section{Chapter three: Methodology}

This chapter mainly describes how the study was carried out in PNG. This section answers the two main questions of how the data was generated and analysed. It also describes the rational for applying specific procedures that were used to identify the problem, select participants, and the preferred method of analysis.

\section{Chapter four: Results/Findings}

This chapter outlines the findings of the research conducted. It provides detailed information in a logical sequence of how the data was analysed based on the methodology that was used to gather information.

\section{Chapter five: Discussion, recommendations and conclusion}

This chapter discusses the data that was analysed. In the discussion phase, it interprets the data and describes the significance of the findings. It also elaborates on what is already known about rural nurse practices and further explains new insights of that problem based on the study. Moreover, it discusses some of the findings including barriers that should be considered in future study and finally is the conclusion which summarises the whole thesis. 


\section{Chapter Two: Literature Review}

\section{Introduction}

The focus of this literature review is to identify what is known about the sustaining factors that motivated rural health professionals, with an emphasis on nurses in the Pacific, to work in such areas, and particularly in Papua New Guinea (PNG). However, because of the paucity of available literature in PNG, and although the focus of this literature review is aimed specifically at the motivations of PNG rural nurses overall, the reviewed literature in this chapter has been expanded to include materials from other Pacific Island countries. It was further supplemented by broader studies into motivation and retention of nurses in rural areas because of the same limitations.

Four different search engines were used to perform the literature review: Google Scholar, PubMed, CINHAL plus, and Scopus. The selected articles will be discussed by the use of main themes. Suitable literature has been purposely selected from all suitable sources published between the years 2005 and 2016 in order to get the most recent research about the factors that motivate rural nurses in developing nations. The Boolean operator was used in PubMed with the key words: motivation, retention, health professionals, rural health, Papua New Guinea, and Pacific Island.

As such, the reviewed literature investigated different factors that have influenced the motivations of health professionals to work in rural locations. The review was primarily reliant on the studies conducted by Jayasuriya et al. (2012) and Razee et al. (2012) because these studies were the only two discovered that were specific to the PNG context. The value of their work is that they provide some evidence to support and underpin the ideas that have contributed to rural health workers' motivations within the PNG context which helps to frame and thematise an authentic PNG rural health narrative. 
Grounded within the literature reviewed, and from an analysis of the data gathered for this research, the following main themes were revealed: extrinsic and intrinsic motivation. The main theme of extrinsic motivation developed from the sub-themes of work environment, which included sub-themes of career development, supervision, incentives, salary and health infrastructures; Whilst, the main theme of intrinsic motivation developed from the themes local environment and personal factors including their sub-themes of accommodation, culture, rural background, family, gender and the generational gap.

\section{Overview}

The two Papua New Guinean studies were particularly focused on the motivation of health professionals to work in rural areas of PNG, and therefore they are most useful for this study. Jayasuriya et al.'s (2012) research focused on the general aspects of nurses' job motivation in rural areas, while Razee et al. (2012) focused on the influence of social factors on job motivation in these areas. The methods used were significantly different from each other. Jayasuriya et al. (2012) used a quantitative approach by using a survey to collect data from 344 nurses who were on a malarial training course. Razee et al. (2012) employed a qualitative approach by employing semi-structured in-depth interviews with 33 selected health workers of different professions.

Their findings vary slightly based on the focus of the studies. Jayasuriya et al. (2012) pointed out that supportive supervision and a suitable work climate play a major role in motivation. Whilst Razee et al. (2012) reported community support, relationships with the community, and effective communication to be the main motivating factors for rural health professionals. These two researchers developed frameworks for their research that generally focused on extrinsic and intrinsic motivational factors. Subsequently, this thesis has used both of these major structures to provide a similar framework, even though demotivation factors as well as motivational ones are also included within that structure; this is discussed further in the methodology chapter. 
Although these two articles have highlighted most of the motivational factors for rural nurses that are featured in this thesis, there was limited literature from PNG to strengthen the studies any further. As a result, literature from variously similar countries worldwide were also reviewed. This included two pieces of research from the Pacific, normally Samoa and Vanuatu. Nine other articles relating to the topic were reviewed from developing countries; namely Sudan, Sierra Leone, Tanzania, Kenya, Zambia, Nigeria, and North Vietnam. However, of greater benefit were those articles that provided insight into rural nurses in both developed and developing countries, and subsequently several research based articles were obtained from Canada, Australia, certain European nations, Thailand, Brazil, Iran, Bangladesh, Nepal, Indonesia, Nepal, Mexico, the USA, and Chad. The inclusion factor for all of the selected articles was a) rural nursing, b) motivational or demotivational factors, and c) retention of nurses.

The searched literature provided some insights into different approaches that were taken by various countries and communities to motivate nurses to select and/or remain in rural areas to practice. These helped to strengthen the rural health narrative. It is hoped that this overall approach can highlight several suitable methods that may assist in the analysis of data and in the presentation of the findings. There now follows an overview of all of the sourced articles arranged under two major themes (i.e. extrinsic and intrinsic motivation).

\section{Extrinsic Motivation}

Extrinsic motivational factors refer to those factors in the external environment that are related to the work environment such as career development and supervision that influence the nurses' motivation in their rural area practices. This motivation is centred on the practice environment and what nurses expect in order to be motivated.

This section focuses on the external environment and how it has influenced the practice of health professionals in rural health settings, particularly the nurses. As suggested by Jayasuriya et al. (2012) and Razee et al. (2012), the main theme is divided into two themes: i.e. working and local environment. These two themes diverged into smaller sub-themes (e.g. career 
development, supervision) that allow for a more explicit explanation of extrinsic motivational factors which are aided by other literature that generally reflects similar themes in their research findings.

\section{Working environment}

There are many work-related factors for rural health nurses that are environmentally based, and the bulk of the selected literature highlighted and discussed significant issues that in this review have been divided into further sub-themes such as career development, supervision, salaries, incentives, and infrastructures. These issues play a major role in motivation especially in the working environment as it applies to rural nursing. Detailed information of each of these issues is outlined in this section including the approaches that different countries have implemented to promote nurses' motivation. These approaches have been explored and then reassessed to suggest possible suitable approaches for use within the PNG context.

\section{Career development}

The majority of health professionals in developing countries are very interested in furthering and advancing their careers. An online survey by Campbell (2015) that concentrated on allied health professionals in rural Australia with the main focus on workers' motivation emphasised that the rural workers training must be one of the top priorities because of the isolation from their colleague which is a barrier for workers to upgrade their skills and practices. Further training encompasses specific skills of learning and it fills the gap between the rural nurses' practice and knowledge (Ajani \& Moez, 2011; Hewision \& Wildman, 1996; Landers, 2000; Rural Health West, 2013; Thu, Wilson, \& McDonald, 2015). As such, study enhancement for rural nurses must be an essential compulsory program to broaden their knowledge.

A lack of skills among clinicians in rural locations is a significant barrier for the improvement of rural health (Kiwanuka et al., 2017; Rourke, 2010; Rural Health Information Hub, 2018; The Health Foundation, 2012; WHO, 2010b; Zhan et al., 2017). To reduce the barrier, rural health workers must get equal training opportunities (Strasser \& Neusy, 2010). This notion is 
supported and further elaborated by Mutale, Ayles, Bond, Mwanamwenge, and Balabanova (2013) who interviewed three staff from 42 health facilities in Zambia, measuring their motivation to work in such areas. They reported that these rural nurses who had gone for career development training had higher motivational score to practice in rural locations. This is because these nurses have broadened their knowledge which has boosted their rural practice. Hence, they are so enthusiastic and are motivated to impart what they have learned with confidence compared to their colleagues who lack such opportunities (Ajani \& Moez, 2011; WHO, 2006). Subsequently, it is vital for the authorities to integrate models specifically tailored for rural workers' training needs to enhance their knowledge while practising in rural health facilities (Msuya et al., 2017; Department of Health in Australia, 2013). It argued that rural health workers were more likely to advance with adequate career planning which will enable them to practice confidently, as highlighted by most of the literature reviewed.

Hence, providing suitable training opportunities for rural nurses is one of the dominant factors for nurse retention in these areas (Strasser, 2001; Strasser \& Neusy, 2010; Rural health information hub, 2018; Rural Health West, 2013). Yet, urban locations are still the preferred environment for nurses to study and enhance their knowledge base. In search of study opportunities, nurses in developing countries therefore often migrate to urban locations (Dussault \& Franceschini, 2006; Kwansah et al., 2011; WHO, 2010b). Migrating from one location to another for study opportunities implies that the preferred area is more accommodating for nursing education (Grujicic, Jovicic-Bata, Raden, Novakovis, \& SipeticGrucicic, 2016; Skillman, Palazzo, Keepnews, \& Hart, 2005). Several authors have noted that it seems apparent that many nurses would believe that the opportunity for them to advance skills and career opportunities lies in the urban health centres, where rural health is not considered as advancing because of the many difficulties in organising study opportunities.

To suppress the urban attraction for education and training, several attempts were made by different countries. For instance, Schoo, Lawn, and Carson (2016) reported two case study scenarios representing two different rural regions in Australia which were identified as region $\mathrm{A}$ 
and $B$. The study claimed that the health facilities in these regions faced similar issues; i.e. physical distance, staff turnover, migration, and other factors, all of which need to be considered for the ongoing motivation of the staff. In region $B$, managers initiated network and inter-professional training among colleagues and engaged professionals in the area of their specific interest to utilise the skills. This practice had motivated and sustained a significant number of health professionals, including many nurses, as a result. Because of the personal development, they were able to retain staff much longer; yet some regions lacked such initiatives, meaning that it had directly demotivated its health workers' interest and indirectly opened the door for its staff to migrate elsewhere in search of other health facilities that provide study opportunities. However, as Macleod et al. (2017) pointed out, the acquisition of appropriate knowledge need not necessarily be physically located. They note that rural nurses in Canada are provided with study opportunities and they study online while practising in rural areas. Both of the above studies argued that career development is possible in the rural sector depending on the environment and availability of required resources.

Despite the notion that nurses in general seem to prefer urban locations as the most suitable option for knowledge enhancement, responsible leaders in developed countries such as Australia and Canada often showed the opposite by developing different initiatives that enabled their nurses to broaden their skills while remaining in rural locations. Yet, it should be noted that adapting such an approach from developed countries is quite difficult in developing countries such as PNG because the health authorities can only do what is possible in their limited contexts to motivate their workers in this way. However, it remains to conclude that more investment in rural health will perhaps allow career development to be part of the rural package.

\section{Supervision}

In addition to adequate attention to career development, good supervision was highlighted in the literature to be a motivating factor. Supervision in the rural health settings is where senior managers guide their workers by providing professional support that enables them to grow 
personally and professionally (Loevinsohn, Guerrero, \& Gregori 1995; Steenburgen \& Mackenzie, 2004; Thu et al., 2015; WHO, 2010a). This basically means that the supervisor must provide feedback on workers' performance and guide them to practice better on their weak areas with an acknowledgement for the effort.

Grujicic et al. (2016) conducted a motivation comparison survey on 396 urban and 436 rural health professionals from four randomly selected health centres in Europe, specifically Central Serbia, and reported that a supervisor's approach towards their colleagues had a significant influence on the health professional's motivation to remain in those areas. They further explained from their study that one of the common supervision issues is the biased practice from the supervisor in terms of good or bad appraisals that are not necessarily based on a true reflection of actual performance and such an approach was noted to be common in rural health facilities. In a parallel manner, a survey conducted in Kenya investigated poor recruitment and retention in the rural health settings of four medical training colleges inclusive of 345 health students, and it reinforced the idea that a significant number of participants reported that poor supervision in rural areas has mostly had a negative impact on their work preferences once they graduate (Mullei et al., 2010).

Moreover, Ojakaa, Olango, and Jarvis (2014), who did a cross-sectional study involving 59 health centres which was comprised of 404 health professionals, argued that besides other external causes, their findings highlighted that poor supervision tends to be a significant issue. They further argued that this practice of favouritism and overlooking one's performance is more common in rural than urban health settings. It was noted in their study that such ongoing issues faced by rural health facilities increases demotivation of nurses unknowingly and they migrate to where they feel supervision is effective. For instance, Grujicic et al. (2016) in their study mentioned that poor supervision in rural areas has resulted in workers migrating elsewhere in most parts of Europe, especially to the urban health facilities. Also, a similar finding was noted by Chimwaza et al. (2014) who conducted a qualitative exploratory study based on health systems strengthening equity and workers' motivation including 84 
participants from various districts and health facilities who agreed that a lack of supervision has already resulted in a high rate of staff turnover for rural nursing jobs in Malawi. This implies that poor or a lack of adequate supervision (in terms of lacking appraisal feedback on job performance) is common in rural locations which disabled the health workers potential to be recognised for future success such as promotion. Macleod et al. (2017) concluded that overlooking nurses' performance without appraising their effort reduced motivation. Nurses feels that their efforts are not valued and they transfer to other locations mostly urban centres where supervision is effective with less bias.

The above discussion demonstrates that adequate career development and supervision motivates workers, and if a manager cannot provide this, it becomes a demotivating environment for staff and they leave. Therefore, health centre managers need to be able to develop their workers in ways that are supportive of their needs.

\section{Medical equipment and social amenities}

Efficient and well maintained medical equipment such as gloves, masks, disinfectants, and others, are an important component for quality delivery of health services despite the location of the practice. Alhassan and Nketiah-Amponsah (2016) conducted a baseline quantitative study in the western regions of Ghana and Greater Accra involving 324 participants with the objective of identifying the rural-urban difference in health workers' motivation to work in rural areas. This study claimed that proper health facilities and equipment have a significant association with overall staff motivation. Sufficient health equipment is vital for workers to prevent occupational accidents and it enables faster diagnosis and treatment of new health problems (Kaur, Hall, \& Attawell, 2001; Thu et al., 2015; Willis-Shattuck et al., 2008). Since health workers are vulnerable to different infectious diseases, it is vital for them to practice with the appropriate health equipment to prevent themselves being exposed. A lack of appropriate health equipment and medical supplies is therefore a deterrent for health workers

to accepting rural offers of employment (Chimwaza et al., 2014; Dussault \& Franceschini, 2006; Kiwanuka et al., 2017; Rural Health West, 2013). That is, a lack of efficient medical supplies 
affects health workers' motivation for the fear of their safety, and as a result, most prefer urban health facilities over rural (Moyimane, Matlala, \& Kekana, 2017; Mullei et al., 2010).

Besides medical equipment, a lack of social amenities, such as water and electricity, is another common issue that demotivates health workers practising in rural areas (Adair-Rohani et al., 2013; CAOD, 2017; Dussault \& Franceschini, 2006; Essendi et al., 2015; Franco, Bennett, \& Kanfer, 2002; WHO, 2018b). Unreliable sources of electricity and water are common in rural areas and it creates an unhygienic environment (Catholic Agency for Overseas Development [CAOD], 2017; Wurie, Samai, \& Witter, 2016). Several researchers note that electricity plays a vital role such as to refrigerate vaccines, provide lights for patient care, to power medical equipment and more, while water enables cleanliness and promotes hygiene. As such, a lack of these essentials has an effect on the crucial function of safe health practices and demotivates the health workers' interest in rural locations as noted. Yet most developing countries health services often fail to provide even basic amenities to the rural health professionals for protection, and this further aggravates the issue (Huttinger et al., 2017; Panagariya, 2014; WHO, 2013, 2014). Furthermore, this lack of basic amenities cripples the health professionals' ability to provide innovative approaches to suit local needs. For instance, Essendi et al. (2015) interviewed nurses in two districts of Eastern Kenya with the aim of identifying the reasons why Sub-Saharan African countries are far behind in reaching most of their health plans and objectives. In their study, it was revealed that the lack of basic amenities such as reliable electricity or water supply is a most pressing issue which has highly contributed to high levels of maternal and neonatal deaths around the world. For any health service to prevent such deaths it needs proper basic amenities and equipment to strengthen its service delivery. Lack of any of these creates devastating gaps in health delivery that may cause many unnecessary deaths (WHO, 2013, 2018a).

Similarly, a lack of health infrastructure and basic amenities does not only affect the workers, it also contributes to the closure of rural health facilities, thereby significantly affecting the recipients of health care (Asante \& Hall, 2011; WHO, 2016). Likewise, Buchan, Connell, and 
Rumsey (2011) stressed that lacking health infrastructure will have a negative impact in the future in regard to rural nurse recruitment to health centres that may close in the near future. This issue was further elaborated by Andrew (2013) who reported about health professionals who moved to centres that have proper health facilities, including basic amenities and avoided ones that were poorly supplied. The WHO (2010a) reinforced that deteriorating health infrastructures and other basic needs therefore decreased the motivation and retention of health staff. Although the infrastructures and social amenities were not the main focus in the PNG studies it is likely that a lack of these amenities create an unattractive rural environment for nurses.

\section{Incentives}

In addition to career development and supervision, Buchan et al. (2012) and the Department of Health in Australia (2013) added that incentives are a major part of the motivational aspects that are purposely planned to motivate and retain health workers in remote locations. Humphreys, Jones, Jones, and Mara (2002), who surveyed 1,400 health workforce based on factors that influence retention in rural Australia, highlighted financial incentives as the main motivation. Financial incentives to work in such areas are often argued to be more of an incentive than ordinary salaries. However, MacLeod et al. (2017) with their recent crosssectional survey focused on motivation which included 3,822 nurses in rural Canada reported that financial incentives are sometimes less effective in nurse recruitment to underserved locations. This is because there are other incentives, such as knowledge enhancement, housing, and working conditions, that are highly likely to be as effective despite poor salaries (Dieleman, Cuong, Anh, \& Martineau, 2003; Hongoro \& Normand, 2006; Rural Health West, 2013). This means that incentives can be financial or non-financial depending on the context of the country.

As such, incentives are provided differently with suitable approach. For instance, Canada provide incentives in financial and non-financial (MacLeod et al., 2017). Australia provides incentives to rural health nurses in the form of significant financial benefits (Humphreys et al. 
2002). Philippines and Indonesia receive incentives through their salaries while Thailand provides a monthly allowance to rural nurses in US dollars (Grujici et al., 2016). Although, some developing countries provide incentives it is highly likely to be very small. Wurie et al. (2016) interviewed 23 health professionals in Sierra Leone with the main objective of investigating the motivational factors for existing rural nurses and noted that financial incentives were provided for motivation but it was a pittance, and thus they did not cater for a family's basic needs. While some developing countries provide incentives, Henderson and Tulloch (2008), review of literature from the Pacific and most of the Asian countries, revealed that most developing countries lack incentives. Unfortunately, poorer nations cannot always offer such financial Incentives. It should be noted that they are given mainly to motivate and retain health workers in developed countries.

The Ministry of Health and Social Welfare Government of Lesotho (MSHWGL, 2010) who reported on the retention strategy for the rural health workforce interpreted a lack of financial or other incentives as a way of negatively overlooking rural nurses' efforts and sacrifices, which has an undesirable effect on workers' morale. They argued that to reduce the staff turnover rate, health sectors, especially in the developing countries, must consider incentives payments to rural nurses as one of their top priorities. Furthermore, when the WHO (2010a) reported on rural nurses' retention rates worldwide with the main focus on developing countries they concluded that those health facilities that provide financial and other incentives face less challenges with health workers' demotivation issues. It remains to add, that although well intentioned, such ideas are often very difficult to realise in developing or poorer nations such as PNG.

Since rural areas are unattractive to nurses, several of the countries health department policies examined for this review treat incentive payments as essential for the rural health profession as a major motivational factor. Indeed, in a rare study that reflected the widespread realisation that PNG lacked sufficient numbers of rural health workers, Henderson and Tulloch (2008) added that incentive payments to rural nurses in PNG are often overlooked, and subsequently, 
health workers such as nurses are considerably less attracted to rural health settings in that country. This revealed that incentive payments are not effective in PNG, because they are often not supplied to health workers on the ground.

\section{Salary}

In addition to the career development, incentives, and supervision, Chimwaza et al. (2014) and Mutale et al. (2013) claimed that salary also plays a major role in rural workers' motivations to go to or remain at rural health postings. Dieleman et al. (2003) conducted an exploratory qualitative study in North Vietnam with 14 participants with the objective of identifying motivating factors for rural health professionals. In their study, it was argued that salary plays a major role in staff motivation apart from other factors. As such, they concluded that providing sufficient salaries to health workers to meet their basic needs will have a positive influence on workers' motivation. As other researchers discovered, meeting this demand usually leads to high retention rates for rural nurses (Humphreys et al., 2002; MacLeod et al., 2017; Ojakaa et al. 2014; WHO, 2010a; Wurie et al., 2016). However, this approach seems impossible for most of the developing countries, such as PNG, with an already inadequate overall health budget where the bulk of that budget is concentrated in urban health facilities (Bolger, Mandie-Filer, \& Hauck, 2005; Connell, 1997). They highlighted that the biased salary payment in favour of urban settings had already attracted more workers from rural locations (especially in developing countries) and what rural nurses earn is insufficient to cover basic family needs (Burchan et al. 2011; Dieleman et al., 2003; MSHWGL, 2010; Skillman et al., 2005). Such practices are a current major issue faced by most countries resulting in the maldistribution of nurses and poor health care services in rural areas. Therefore, the studies noted above all indicated that rural nurses' salaries must be increased to motivate and attract more workers.

The above discussion is supported by other broader, but applicable research. For instance, Willis-Shattuck et al. (2008) revealed through their systematic study based on rural health workers' wages in developing countries that nurses in urban areas of developing countries earn higher salaries than their rural counterparts. Furthermore, Baernholdt and Mark (2009) and 
Skillman et al. (2005) conducted similar surveys in the United States that focused on nurses' work environments with job satisfaction and revealed that paying rural nurses low salaries is perhaps a common practice because of the fewer technological complex procedures that the rural nurses perform (due to less equipment and small health facilities compared to the work of urban nurses). Hence, treatment, such as unequal salaries that favour urban health workers is a bias that creates an unfavourable financial environment for rural nurses. Nevertheless, this approach has been criticised by Mullei et al. (2013), Burke (2015), and Skillman et al. (2005) who argue that rural nurses should be eligible for higher salaries because of their sacrifices in isolated locations compared to their urban counterparts. Furthermore, Darkwa, Newman, Kawkab, and Chowdhury (2015) conducted an in-depth interview with 21 participants based on factors influencing retention of nurses and doctors in rural health facilities in Bangladesh. Based on this study they argued that most health workers serving rural health facilities deserved higher salaries because of certain factors such as, poor working conditions, higher workloads, and lost opportunities for extra income from private practice. In any event, and especially in the case of PNG, it may be argued that because over $85 \%$ of the population is in rural areas, the rural nursing salary should be higher to encourage nurses to work there.

Swanson's (2013) report highlighted the lack of health professionals in rural PNG and mentioned that not only do the urban settings offer higher salaries, but that Non-Government Organisations (NGOs) and companies also offer more pay, and consequently some health workers are attracted to work for those organisations. For instance, Buchan et al. (2011) noted that high salary payments from NGOs and companies has negatively affected the recruitment and retention rate of the government related health facilities particularly in rural areas. This was further supported by Andrew (2013) who emphasised that the main factor related to the declining of the workforce in government related health facilities in PNG is low salary rates for health professionals. Indeed, as Swanson (2013) reported, PNG has lost many of its qualified health professionals to the mining sector for a better salary rather than a commitment to health work elsewhere. Most researchers, and specifically PNG researchers, who examined the 
situations for rural nurses appear to agree, i.e. that such nurses should receive a higher salary than their urban counterparts due to the practice and population demands.

To increase motivation, MacLeod et al. (2017) explained that rural areas of Australia have suppressed any financial demotivating factors by increasing the salary of those working in these areas, which has led to higher numbers of nurses being recruited and employed. This is thought to be because of the enhanced career development and financial incentives, which are then the main attraction over the problem of the isolated rural location. Similarly, Humphreys et al. (2002) reported that rural health workers in Australia are highly paid, i.e. considerably more than their urban counterparts, which has increased their motivation and has attracted more urban health professionals. However, the literature reviewed indicated that such practice is quite difficult in developing countries, and some staff move to urban locations or NGOs/private enterprises for a higher salary. As a result, rural workers in PNG are faced with difficult choices and are less attracted to government facilities; many therefore prefer working for NGOs or companies in search of high salaries.

As noted, incentives and high salaries are provided to motivate workers in rural locations. However, from the findings of the two PNG studies alone, it seems very difficult to address health workers' financial issues in PNG because there are many related issues to consider such as issues related to intrinsic factors.

\section{Intrinsic Motivation}

Intrinsic motivation is the internal motivation of the individual nurses about how they are personally challenged by working in rural locations. These are mainly related to the nurses' own feelings, experiences, and their expectations of motivation.

This category will focus on the internal environment that motivates health professionals to work in rural settings as opposed to working in urban ones. The internal environment is the 
nurses' personal motivating factors and therefore includes local environment and personal factors.

Two main themes have been revealed in literature, namely local environment and personal factors. These two themes have different sub-themes; accommodation and culture are related to the individual within the local environment, whereas personal factors are comprised of rural background, family, gender and age. This section of the literature review will investigate these sub-themes in detail focusing on the internal environment and will provide details on how it affects health practice and practitioners in rural locations in order to highlight motivations or otherwise that are outside of the extrinsic factors discussed previously.

\section{Local environment}

Jayasuriya et al. (2012) and Razee et al. (2012) highlighted accommodation and culture as the main causes of motivation for rural nurses in regard to the local environment. What follows is a further discussion taken from assorted articles concerning these two important factors and the influence that they have on rural nurses, especially those in developing nations such as PNG.

\section{Accommodation}

Research has shown that one of the foremost problems for retaining qualified health workers in regard to environmental issues seems to be accommodation issues. Accommodation plays a vital role in staff retention because rural areas of developing countries are under-developed and isolated (Lehmann, Dieleman, \& Martineau, 2008; Mullei et al., 2010; Rourke, 2010; WHO, 2009, 2010a), Subsequently, there is more likely to be less accommodation, and even then, the rental accommodation provided will probably not meet the standard and expectation of nurses (MSHGL, 2010; Theime \& Weber, 2014). For this reason, the WHO is supportive of the idea of providing accommodation to rural health workers. This notion was elaborated upon by Okello and Gilson (2014) when they mentioned that suitable accommodation is highly correlated with staff retention, particularly in middle- and low-income countries. Their argument was based on a systematic review of 31 articles inclusive of low-, middle-, and high-income countries. The 
articles reviewed were taken from between the years of 2003-2013 and focused on rural health workers' motivations to work in such areas. Although there were other factors identified which had significant impacts on motivation, they found that accommodation was one of the most pressing.

Moreover, Wurie et al. (2016) with their study discussed previously, concurred that a lack of accommodation in rural areas in developing countries is an issue for health workers who seem to reject rural offerings for urban settings. This has a great deal to do with their search for suitable accommodation, including suitable amenities as previously discussed. In addition, Jayasuriya et al. (2012) and the media outlet Loop PNG (Expanded accommodation for, 2017) expressed that an NGO's related organisations such as churches or companies provide accommodation for their employees, but government organisations were far less accommodating, particularly in PNG. As such, many of the qualified nurses in rural areas in PNG had already moved to work for companies or religious bodies, including church related health facilities, in search of provided accommodation (Swanson, 2013). These researchers identified accommodation to be a particularly important issue in rural locations. Thus, all authors in this review suggested that the provision of suitable accommodation to be a most important aspect of retaining nurses and other health workers in rural locations.

It is mentioned in this thesis that NGOs such as the churches and companies in rural locations provide their health workers with accommodation or rental allowances when compared to their counterparts in government related health facilities. Yet while the Papua New Guinean health sector also applies such an approach of providing accommodation and rental allowances, it seems to be more specific to some of its workers such as senior medical practitioners in rural locations, but not others, such as nurses.

Overall, research shows that providing either the rental allowance or accommodation was effective in increasing the motivation of health professionals in rural areas of PNG. Therefore, in order to motivate nurses towards rural practice, there must be a consideration for 
accommodation as a key factor. In addition to accommodation, the literature revealed that culture of the community is also a significant motivating factor.

\section{Culture}

The United Nations Educational Scientific and Cultural Organisation (2009), specifies that culture is the very substratum of all human activities and a vast majority of developing countries are strongly multicultural, as is most certainly the case in PNG. In developing countries, cultures are preserved and nurtured and this is common in rural areas. Hence, it is a challenge for nurses in rural PNG with over 800 different cultures.

Razee et al. (2012) conducted their semi-structured in-depth interviews involving 33 participants in rural PNG. This study which was focused on the social aspects of different categories of health professionals based on three different provinces in PNG highlighted that the community participation and adapting to their lifestyle is the greatest social influence on health workers' motivation to remain in that community. Subsequently, to avoid intercultural encounters, health workers must be open to changes by involving themselves with the community and accept cultural beliefs that differ from their own. This means that it is very important to incorporate cultural beliefs into medical practices, whenever possible, to provide appropriate care that satisfies people and will enable communities to respect workers which will encourage a bond and a good relationship. As a simple rule, this approach appears to present few problems, but in practice, it may present difficulties for the health worker whose own cultural (and professional) background is often significantly different from that of the recipient of care.

This is especially the case in PNG, where it should be recalled, there are over 800 different cultural groups and languages. Yet, if treatments are to be effective, it is clearly of vital importance for a health worker to understand the community's culture in different aspects and what matters to them such as beliefs, ethics, values, and heritage (Bourke \& Sheridan, 2010; Fowler, 2009; Kiwanuka et al., 2017; Thu et al., 2015). This is only achieved through 
socialisation with surrounding communities which demands that the nurse have fair knowledge of different local cultures and enables their practice to be culturally appropriate (Jayasuriya et al., 2012).

The above argument is a major issue in many developing (and developed) countries. According to her study on cultural barriers in health care based on Bolivian cultures, Fowler (2009) argued that having different views and beliefs of medicine between health workers and the community can cause difficulties in the effectiveness of health service delivery. Moreover, Dieleman et al. (2003) and Polaschek (1998) both mentioned that such misunderstanding often leads to a community's preference for traditional treatment methods rather than incorporating traditional and modern medical techniques, as is often attempted in PNG. Hence, it is clear that health workers such as nurses who socialise with the community, and successfully bridge cultural gaps, are empowered to practice in a culturally safe and respectful way, thereby nurturing different cultural identities (Smith, 2013). Whilst a similar approach is applicable in PNG, it can be concluded that it is of vital importance to firstly introduce the mores and norms of people from different cultures to the newly recruited health worker to avoid any cultural confrontation at their initial phase of practice. In having a reasonable knowledge of culture, the health workers should therefore respect the traditions and cultures of the local populace, and value and respect them in return. This should promote social harmony between the health worker and the local population which will subsequently have an impact on their motivation to remain in the rural area. Besides local environment the impact of personal factors on the nurse must be considered.

\section{Personal factors}

Jayasuriya et al. (2012) and Razee et al. (2012) revealed that health workers' job motivation in rural areas depend on the quality of each individual's life. The literature reviewed highlighted three main personal factors that have contributed to rural job motivation in this regard. These include age, family, and gender of the health professionals, as outlined by the two main PNG studies and supported by other literature. 


\section{Rural background}

Jayasuriya et al. (2012) and Razee et al. (2012) indicated that PNG seems to have a great number of local health professionals, particularly nurses, who practice in their own respective communities. Buchan et al. (2012) and MacLeod et al. (2017) agreed that the rural background of the nurse or whomever has a strong positive impact on the health workers' motivation and retention. This notion was strengthened by Mbemba, Gagnon, and Hamelin-Brabant (2016) who argued that rural lifestyle, origin, and background are highly associated with the motivation and retention of rural born individuals in regard to those personal factors that are attributed to their work in these areas. This was based on their systematic review which was focused on the factors that influence motivations of rural and remote based health professionals of several developed and developing countries. From this study, it was noted that a rural origin had a significant impact on the workers' motivation besides other pressing factors. Hence, most rural raised professionals often work in rural settings, particularly in developing countries (Mullei et al., 2010). This means that health professionals with rural backgrounds have less chance of rejecting rural offers and are indeed more likely to be attracted to them if the work is within their own tribal or regional areas.

Similar to Mbemba et al. (2016), Serneels et al. (2010) conducted a cohort study in Rwanda and Ethiopia with 42 nursing and medical student participants. This study set out to identify those factors that measure intending health workers' preferences for rural locations to assist policy development. In this study, it was revealed that health workers who had a rural background supplied one of the two main motivating factors for their choosing rural areas to live and work. Moreover, Blauuw et al. (2010) whose research on the policy interventions grounded on their cohort longitudinal study in Kenya, South Africa, and Thailand with 300 recently graduated nurses argued that despite lack of policies specific to rural nurses, those workers with rural backgrounds has high chances of choosing rural locations. Rural background had a positive influence on students' preferences in job applications. 
Overall, the different studies noted above, from various countries, identified nurses with rural backgrounds to have considerably higher motivation to work in rural practices. Thus, all studies claimed that recruiting professionals with such criteria help to prevent nurses from being demotivated. The literature therefore supports the idea that it is workable and desirable to encourage local nurses to return to work in their own local communities; as previously noted, an examination of the literature revealed that this approach has obviously worked for many developing countries such as PNG. This is especially so in PNG because it is a very strong familyand community-oriented society, therefore, most Papua New Guinean health workers prefer serving their own communities if they have the opportunity. Hence, recruiting workers back to their home regions should lead to less staff turnover, increased motivation, and a further decrease in nurses' demotivation in these areas. The importance of family, and the security and happiness of one's family is clearly an important element in this conclusion.

\section{Family}

It appears from a review of appropriate literature that families play a major role in influencing health workers' choices regarding their working location. Buchan et al. (2011), clarified that the concept of family in the Pacific region is heavily based around the importance of extended and immediate families, therefore the satisfaction of working in rural areas often depends on both. As such, work location for health professionals is often decided by their family (Kiwanuka et al., 2017; Lehmann et al., 2008; MacLeod et al., 2017; Rourke, 2010; Rural Health West, 2013). To increase motivation, a recent study in Canada by MacLeod et al. (2017) recommend that rural areas should recruit health professionals as couples, whenever possible. This is because having couples of similar professions seems to be the appropriate approach when considering family because both will have a job, and both will contribute to local health needs. Employment for both spouses in rural areas prevents migration (Jayasuriya at al., 2012). This means that considering couples of health professionals could be one of the foremost recruiting criteria for rural nurses. 
Razee et al. (2012) justify that one of the reasons for health workers choosing urban over rural locations is to have easy access to meet the needs of family, especially the children. This is underpinned by the notion that some rural job turnover by health professionals is likely to be because of the need to further their children's education (Buchan et al., 2012; Kiwanuka et al., 2017; Witter et al., 2017). That is, a rural lifestyle may be adequate when the children are younger, but when they grow older and require higher educational support, the family may move to an urban area. Based on their views, it is suggested that a family's obligations, status, and ethnicity and the educational needs of the children must be included in the recruitment criteria and be considered when offering them a job to avoid excessive staff turnover.

Based on the research findings noted above, it seems that recruiting local health workers or staff who are married with children to work in their own local health facilities will have higher motivation and therefore a higher chance of retention in that area in PNG. As such, it would better to prioritise the local workers and spouses of locals should in the recruitment process which will result in having less turn over, decrease the demotivating factors, and have more workers. Yet, the use of families of health workers has its limitations - children grow older and need higher education, couples seek greater support networks, and eventually many rural health workers simply grow old and are no longer able to perform what is often exhausting and strenuous health related work in rural areas.

\section{A generational gap}

Swanson (2013) argued that the former Health Minister in PNG reported that the government needs to promptly respond to the issue of an aging workforce in the rural areas of PNG or the rural health system will soon collapse. This was further reinforced by Razee et al. (2012) who in their study on work influences highlighted that aged persons in the health workforce, particularly in the rural areas of PNG, are not replaced when they retire, thus the staffing in these locations is often below the required level to achieve the country's health goals. This is currently a significant concern for PNG (Global Health Workforce Alliance, 2018; Kora, 2016; Nursing Matters, 2015; The World Bank, 2013). The shortfall in the workforce obviously has 
caused serious constraints on implementing the plans and policies of the NDoH which in turn affects the rural health and the health care providers.

Furthermore, in their cross-sectional quantitative study that included 200 health workers in both the urban and rural settings in Nigeria, Ebuehi and Campbell (2011) further strengthen this notion by stating that the age of individuals has a significant influence on rural job motivation; that is, older health professionals appear to prefer rural locations over younger ones. As this was one of the common cases in most of the developing countries, Baernholdt, Yan, Hinton, Rose, and Mattos (2012) investigated further through a comparison survey of aging adults in rural and urban locations to identify their health and nutritional status. Their findings highlighted that most of the older adults migrate to rural areas or refuse to move to urban locations because of the friendly and more caring environment for aging residents. Although this survey does not include health professionals, it reflects why most aging nurses are found to be working in rural environments such as PNG.

PNG is one of the countries that was reported to have most of the aging health professionals in rural locations. For instance, Swanson (2013) reported that rural health centres in PNG comprised of a vast majority of aging health professionals. Moreover, the Asian Development Bank (2014) under the rural primary health division outlined that the majority of the health professionals working in the rural areas of PNG are over 40 years old. This is most likely related to the previously mentioned effects of family ties, tribal affiliations, and acceptance within a given community which confirms the findings of Baernholdt et al. (2012). Indeed, as Jayasuriya et al. (2012) explained, those health professionals who are at the active stage of development, such as young professionals, seem to have high turnover rates for rural jobs compared to their elder counterparts. This was also indicated by the WHO (2016) and Swanson (2013) who reported that PNG has fewer young health professionals serving rural locations than they require, and that older health care workers eventually struggle to provide the necessary health care practices under difficult conditions. More aging health workers in rural areas clearly 
insufficient and constrains essential health care delivery in a country where over $85 \%$ of the population lives rurally.

Most of the above studies indicate that overall, older health professionals are stationed in rural locations, whilst younger ones seem to favour urban locations. They further state that if such an approach remains unsolved it will have negative impacts on rural health because younger workers are needed to maintain continuity. Thus, it was suggested that the health sector address this by having sufficient numbers of young workers in rural locations; this is a highly pertinent notion when applied to the situation in PNG. However, such a manoeuvre is not without additional difficulties, and in a country such as PNG, this is complicated further by gender issues.

\section{Gender}

Gender plays a very important role in workers' choices for rural locations. Jayasuriya et al. (2012) pointed out that working in some rural areas of PNG as a female can be very challenging. This is often because of geographical remoteness where nurses will have to travel (by foot) for hours to reach the health facilities or deliver services to the surrounding villages, often at a significant cost to their family obligations, which for women in PNG, are extensive. This means that less time is spent with the family due to the long-distance travel to deliver health care, and potential problems within the relationship between a female nurse and her husband.

Moreover, travelling in remote areas is risky for females especially in underdeveloped countries such as PNG with the existing issue of frequent reports of violence against women in that country (Human Rights Watch, 2015, 2016; Women with disabilities, 2013). As revealed by Ojakaa et al. (2014), a similar issue is faced in Kenya and undoubtedly by most developing countries. Their study found that rural employment in Kenya has been a greater challenge for females compared to males and that security at home and in the workplace is much riskier for female workers. It is obvious that the challenges that female workers face in rural areas are more than their urban counterparts (Buchan et al., 2011; Jayasuriya et al., 2012; MacLeod et al., 
2017) because of the ever-present danger of violence and rape. Thus, there appears to be a reliable argument that females fear practising in rural locations in many developing nations, and many will therefore only work in the relative safety of urban centres.

Buchan et al. (2011) explained that as a result of females neglecting rural offers only males are often considered for rural practices. They based their argument on the Vanuatu Government's Health Department aggressively targeting males for rural locations. This approach was taken after it was noted that most of the females, compared to males, that are sent to rural areas often refuse the offers because of the geography. As such, the recruitment of rural health professionals is male biased to avoid inconvenience. However, Jayasuriya at al. (2012) mentions that such a practice is quite difficult in PNG because this health sector especially the nursing profession remains largely female dominated. Yet their studies revealed that females seem to be less motivated to work in rural practices meaning that most males are to be found in rural areas compared to females. This implies that although the chances of assault and violence may be reduced, males who are nurses are often given risky working assignments in the remotest parts of PNG, and for some, this would be a task that was too demotivating; they also are eventually more than likely to seek work in urban areas.

Previously, some evidence has been presented to suggest that recruiting couples who are trained in health care may offset gender balance and also provide sufficient nurses to meet local health needs. However, it may still be possible to employ both single and married female nurses in remote rural areas under the right conditions. For instance, MacLeod et al. (2017) maintains that in countries such as Canada, female employees such as nurses who are given the opportunity to work in their own isolated rural communities are sufficiently supported by that community and therefore motivated and have higher retention rates than others. According to the study, it may be possible to apply the concept of the Canadian experience to the PNG context because of the wantok system practice in the country where the female's safety is guarded by friends, family, and relatives. 
To conclude, intrinsic motivation is related to the personal lives of health professionals outside of extrinsic motivational factors. It is apparent that poor accommodation and cultural barriers do influence health workers' motivation regarding the suitability of the rural environment. However, it seems obvious that health workers with rural backgrounds have higher retention rates in rural areas despite some of the different conflicting issues as noted in this chapter. Family choices also add to the motivation to work in rural areas, as well as factors relating to the generational gap where older workers prefer rural settings over urban while younger staff find urban areas to be more lucrative. Yet it should also be noted that the gender issue has a negative impact on females choosing rural locations. Despite the different factors related to intrinsic motivation, a rural background is more likely to have a significant impact on nurses' motivation if it is critically considered. Therefore, developing countries like PNG which are in critical need of rural nurses must consider prioritising nurses with rural backgrounds to practice in their own community. Applying such an approach could reduce staff turnover and sustain nurses, whilst at the same time strengthening commitments and services for members of the local population.

\section{Conclusion}

The current problem faced by rural locations in most countries, and in PNG in particular, is the shortage of adequate health care personnel. A large number of the reasons for this shortage are explained under two main sets of influencing factors, i.e. extrinsic and intrinsic. The problem of maldistribution in favour of urban locations seems to be inevitable, and as mentioned, overlooking these significant issues will result in future closures of Papua New Guinean health centres and rural health will eventually collapse if nothing is done to motivate health professionals; PNG is no exception to this need. Yet, there are ways that this issue may be alleviated, and regardless of the fact that many rural health facilities have already been shut down in developing countries such as PNG, there may be ways of retaining rural staff that have never been fully explored. In the next chapter, I will discuss the methodology and methods that were employed in my research that examined the ways that nurses are motivated (and/or demotivated) to remain or leave rural health care areas in PNG. 


\section{Chapter Three: Methodology}

\section{Introduction}

This study aims to explore and describe the sustaining factors that motivated rural nurses in Papua New Guinea (PNG) to remain working in rural areas. As stated earlier, nurses in rural areas play a vital role in the health service delivery to a rural population, and this is especially so in countries such as PNG. However, nurses and other health workers are very limited in number in such areas when compared to urban locations. Indeed, a recent study by Watson, Sabumei, Mola, and Ledema (2015) reported that a severe shortage of nurses in rural areas of PNG is one

of the contributing factors to a high mortality rate in those particular locations. It remains obvious that there is a significant ongoing decline in nurses in rural locations where the health status is worst, and this is a major concern for the country. Therefore, this study seeks to identify causes impacting on sustaining and supporting rural nurses. To identify those causes, the study focused directly on rural practices and their challenges, such as the attractiveness of the working environment and personal motivational issues. By identifying these causes, this research also sought to articulate feasible solutions to address any significant issues that emerge from the data. In order to identify the sustaining and sometimes because of the nature of the topic, the non-sustaining factors, a qualitative descriptive-exploratory method has been employed.

This chapter is discussed in two parts; the first part focuses on qualitative research processes, and more specifically on the interview process and the rationale for the overall design of the study. Part two focuses on the steps involved in data collection.

\section{Aim of the Study}

The study aimed to identify those sustaining factors that enable nurses to work in the rural areas of PNG. Since less is known about this topic, particularly in PNG with its exceedingly limited literature, the study further explored and described the situation of why nurses favoured urban locations over rural ones to practice. Having this objective, and faced with a 
dearth of suitably specific literature, a descriptive-exploratory method was to be the appropriate methodology.

\section{A Brief Introduction to the Research Approach}

Research is an ongoing exploration that plays a vital role in building capacity in any given society. This means that research enables researchers to identify issues and solutions, where they can confirm or refute a hypothesis or grouping and develop or refine a theory to see whether it either predicts or controls certain events (Nebeker et al., 2015). There are two different major approaches to any research study: qualitative and quantitative. These two approaches are significantly different from each other regarding the types of information that they collect and the methods that they use to analyse the raw data. For instance, qualitative research is an excellent approach when in-depth interviews, focus groups, projective methods, case studies, or pilot studies are being considered, i.e. it gathers data using non-numerical values.

On the other hand, quantitative research is an excellent approach when considering any fundamental connections between empirical observations and quantitative relationships. As such, quantitative research methods rely on the statistical analysis of numerical data to produce results that can be generalised to some larger population. In any research study qualitative and quantitative approaches are either used separately or combined depending on the nature of the study. Both approaches have been successfully applied to previous studies of nurses in PNG (Jayasuriya et al., 2012; Razee et al., 2012) but the exploratory and descriptive nature of this study strongly indicates the need for a qualitative approach. This study therefore employed a qualitative approach that uses data gathered from in-depth interviews and relates the findings through thematic analysis and description.

\section{Qualitative Research}

Qualitative research is an effective method used for obtaining information that is based on values, behaviours, and opinions (Mack, Woodsong, MacQueen, Guest, \& Namey, 2011). Such a 
study involves the investigator who becomes the tool for both the data collection and data analysis (Woods, 1997). This means that the researcher often participates in the research by being at the site of the study and interacting with the participants to collect their data. Subsequently, the information provided by the qualitative approach is rich in nature because the participants are given the opportunity to elaborate on their views.

Some of the main approaches to qualitative research studies are grounded theory, phenomenology, and descriptive-exploratory research (Woods, 1997). Through a descriptiveexploratory approach, this study employed the technique of formal qualitative research through the use of data gathered at interviews. In such interviews, the emerging data may be of sufficient exploratory depth to allow detailed descriptions of the topic to emerge. Interviews can be either semi-structured or unstructured, and may include individual interviews, observations, and focus groups. For the qualitative approach, the sample size is usually small with in-depth information. As such, this study utilised a sample of 10 semi-structured interviews. These interviews were than analysed against the current literature to develop the themes used to explain the analysis of this research.

\section{The Descriptive-Exploratory Approach in this Study}

Descriptive-exploratory research is one of the most common methods in research studies where there is limited information regarding a specific phenomenon (Sousa, Driessnack, \& Mendes, 2007). This approach is often used to identify problems that have not been studied clearly and provide possible solutions to the issue. The result of such a study is to provide significant insights into a given situation, and although obviously not generalisable, it can

provide possible thematised explanations that may assist in future research. More importantly for the purposes of this study, such an approach may greatly assist in planning for the delivery of nursing care in rural areas of PNG. As there is very limited literature on the work of PNG nurses, and no discernible previous research on this topic at all in PNG, this approach was considered necessary in order to at least open up some research possibilities and illicit common themes and ideas from the interviews. 
Exploratory research through a formative qualitative study is very useful in countries such as PNG where there is no or hardly any available literature. In part, this is because PNG is one of the least explored countries in the world, in research or otherwise (Reaching for the frontier, 2011), but also because of limited infrastructure, shortages of trained researchers, and weak research capacities. This means that many of the factors that have negative impacts on the livelihood of Papua New Guineans remain undiscovered or under-researched. Nursing research in PNG is essentially a very rare phenomenon, and even rarer when considering rural nursing, e.g. an extensive search of the literature showed only two available studies (Jayasuriya et al., 2012; Razee et al., 2012) in regard to rural nurses' motivation in PNG. Lacking such vital literature directly impedes the policy development for specific areas such as rural health because of insufficient evidence.

Although there are nationally established and well-intentioned health policies, access to health services in PNG remains a major problem. A common cause of this problem is poor access and a lack of nurses in rural areas; in part this is one of the impacts of decentralisation and a lack of specific policies for existing rural nurses (Kolehmainen-Aitken, 1992), the existing policies are not specific to rural nurses. Nevertheless, a set policy is needed for rural nurses' motivation to reduce the nurses' maldistribution issues and to encourage more nurses to work in the rural areas of PNG. As is nearly always the case with health policy, the need for the development of such a policy will only be highlighted through research.

Thus, for the above reasons, this descriptive-exploratory study was conducted with nurses practising in rural locations. Methods used in the study allowed for further exploration into the motivation of nurses to work in rural areas where less is known about their needs. Subsequently, the description of the study emerges gradually following an innovative exploration which serves to arrange the results based on the highest motivating factors to the lowest according to the sequences to fit the explanation. Hence, this study investigated nurses' motivation to work in rural health settings. It is hoped that this study will provide insight into 
some factors that affect rural nurses' motivation and will further guide the policy makers to develop specific policies which will sustain rural nurses in PNG.

\section{The Interview Approach}

There are different ways of collecting data for a descriptive-exploratory research. The appropriate method will depend on the aim of the study and the thesis question. For instance, this study's aim is to identify sustaining factors for rural nurses, meaning it is specifically trying to explore the motivating factors for rural nurses. Following the interviews, the feedback from these participants was analysed to identify the causes of nurse shortages in rural health settings, and to also consider those factors that sustained nurses in such areas. Such a study needed carefully worded questions as prompts to guide the participants in order to extract the needed information. Clearly, this type of approach requires open-ended questions within a semi-structured approach. Open-ended questions explore the topic by allowing the participants to provide rich and detailed information and further provide opportunities to both the researchers and participants to diverge and investigate the idea in detail (Gill, Stewart, Treasure, \& Chadwick 2008; Mack et al., 2011). There were six focused questions that were asked in the interviews (Appendix 3), and the discussions that emerged were divergent in their exploration of the details needed to capture the responses that answered the thesis question. The interview questions were focused based on the nurses' work experience; such as the challenges that they faced in health service delivery and how those challenges were overcome, why there are very limited nurses in such areas, and how working in rural areas has impacted their personal lives. After the interview, each nurse was provided with the opportunity to ask questions.

The aim of the research was to enable nurses to openly and freely express their thoughts without the fear of being caught and this is possible through maintaining the confidentiality of participant information. The interviewees were given a consent form and a confidentiality agreement to read and sign and were made aware of their rights to withdraw. This is because the selected participants must remain anonymous in order for them to revel in-depth 
information without fear (Appendices 1 and 2). Moreover, the information provided was kept confidential between the researcher, supervisor, and the participant. In addition, the researcher wished to provide each participant sufficient time to openly express their views and opinions without fear of loss of anonymity. Such a method of data collection is commonly found in semi-structured one to one interviews.

\section{Data Analysis}

Thematic analysis was the method used to analyse the research data because of its suitability for the task. Braun and Clark (2006) defined thematic analysis as a systematic method used for identifying patterns, analysing and reporting them in an accurate fashion. Thematic analysis is commonly used in qualitative analysis because of its flexibility and accessibility (Braun \& Clark, 2012). This method of data analysis can be used by any qualitative researcher who is not an expert, meaning this approach is appropriate for novice researchers, or it can be used to analyse data by people who are not trained researchers (Onyx \& Small, 2001). On use, it was found that thematic analysis successfully described the collected data in detail. Moreover, Braun and Clark (2014) explain that thematic analysis is a very useful qualitative approach for investigating the policy or practice arena. Since such an approach to analysis has been recognised for policies and practices, the collected data were analysed and discussed to propose some recommendations for specific policies which need to be developed for rural nurses' motivation.

Braun and Clark (2012) list out six main steps to a thematic analysis. They are: familiarise with the data, generate initial code, search for the theme, review, name the theme and produce a report. The researcher must firstly go through the raw data (such as interview transcripts). This step further involves a critical analysis of the data by asking different questions to help figure out what the data actually means. Repeatedly going through the raw data allows for familiarity as well as noticing some of the things which are relevant to the research. In step two, the data are coded using line by line or sentence by sentence open coding. In this, the data that is relevant to the aim of the research are extracted and coded systematically according to the 
overarching code. The portion of the sentence that identifies the code name is highlighted and written besides each sentence. Here, there will be some data that the author may be unsure about which also needs to be coded.

The third step is reviewing the codes to identify any similarities and overlaps that may have emerged from the codes. An example of this phase is shown in Figure 7. This diagram is from Braun and Wilkinson (2003) who researched the vagina and is based on women's talk (as cited in Braun \& Clarke 2006). The diagram shows different related codes combining together to develop five main themes.

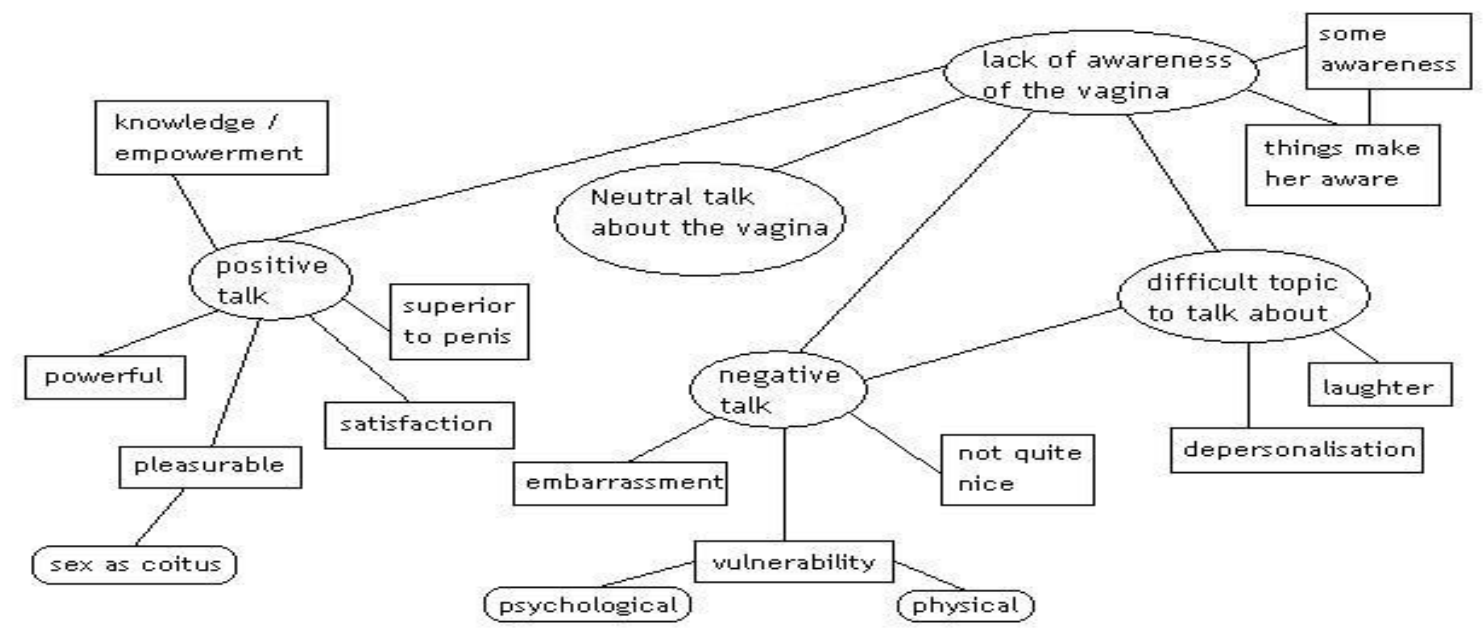

Figure 7. Initial thematic map showing five main themes

(Braun \& Wilkinson, 2003, as cited in Braun, V. and Clarke, V. (2006). Using thematic analysis in psychology. Qualitative Research in Psychology, 3(2), 77-101).

Moreover, a quality check is done by reviewing the coded data where the themes are checked against the data to see the relationships. In this phase (i.e. phase four) some of the codes which are not related with the theme may be discarded or relocated to other themes.

The fifth phase is where the analysis is shaped and should provide the meaning and the interpretation as shown in Figure 8. Finally, the themes are arranged to tell a story. 

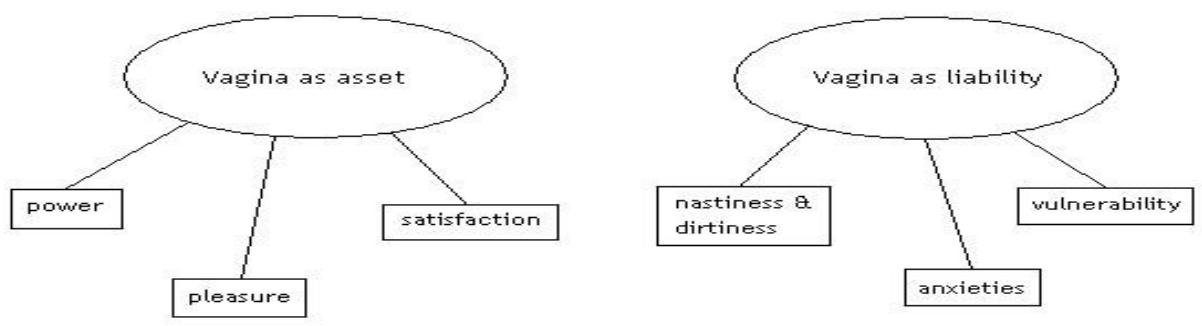

Figure 8. Final thematic map, showing final two themes

(Braun \& Wilkinson, 2003, as cited in Braun, V. and Clarke, V. (2006). Using thematic analysis in psychology. Qualitative Research in Psychology, 3(2), 77-101).

This thesis is heavily reliant on the interviews due to the limitation of literature concerning nurses in rural areas of PNG. The research conducted was analysed thematically to identify the underlying issues of the nurses' motivation in rural areas. In addition, its data was analysed using an inductive approach to identify the main causes of nurses' motivation in rural areas. It is hoped that this study will help the policy makers to ensure most of rural nurses' motivational factors are included in their policy development which if developed and acted on will more likely push the health sector forward in achieving its eight objectives in the 2010-2020 national health plan as discussed previously in the introduction chapter. The outline of the study conducted is discussed below.

\section{Data Collection}

The application of a descriptive-exploratory study was done in collecting the required data. This section provides the steps which were used in the study to gather data.

\section{The participants}

There are two groups of nurses in PNG, one called community health workers (CHW) and another called registered nurse (RN) who are present in almost all health facilities in the 
country. $\mathrm{CHW}$ are nurses with a certificate in basic nursing skills. A job description for a $\mathrm{CHW}$ is typically nursing care oriented. Whilst, the registered nurses are those nurses who have undertaken intensive nursing education and have a diploma or a Bachelor's degree in nursing. The nursing officer oversees the nursing activities in health facilities such as, nursing care, intravenous drug administration, patient assessments and reports. However, the lack of qualified nurses and doctors in rural locations has meant that the CHWs perform beyond their scope of practice, such as inserting intravenous cannulas and admitting patients which is the function of doctors or qualified nursing officers.

While the thesis acknowledges the work of other health professionals, the CHWs and nursing officers with over two years of rural practice are the major focus in this study as primary health care providers. They were chosen because it is these nurses who offer a vast part of the health care in PNG's rural areas. A total of 10 randomly selected participants with over two years of rural work experience took part in the study, three from church health centres and seven from government related health facilities. The study comprised of three males and seven females, which is incidentally a rough reflection of nurses' gender division in PNG. However, a few of the participants had served in rural areas for over 20 years. The age range of the participants was 30 to 60 years.

Almost all of the participants were local nurses, some of whom had worked in the rural health facilities since they graduated from college, meaning that they had spent all their working career stationed in one location. Few had moved from urban to rural locations to serve their own communities. However, those in church related health facilities often moved from one remote location to another depending on the demand, staff shortage, and the location of their posting.

\section{Procedure for recruiting participants}

Two rural facilities in one of the provinces in PNG were selected for the purposes of the research. These health facilities are governed by two different organisations: the government 
and the church. The government related health facility is situated in the main centre of the district where most basic services are available, whilst the church run health facilities are located in the remotest part of the district where access to basic services is limited. These hospitals were chosen to represent what it is like in PNG in terms of health facility distribution in rural areas. For instance, most of the government related rural hospitals in PNG operate are built in the main centre of the districts where there is easy access to basic services. Whereas, the church run health facilities function in the remote parts of the districts.

Before the study, the hospital managers of different health settings were each provided with an information sheet. The ethics approval was given verbally by hospital managers because of its rural context in lacking equipment for written approval. However, using an information sheet (appendix), the respective clinical managers spoke to their nurses individually, seeking their permission to participate. Those nurses who showed interest had their names recorded and these were dropped into two separate labelled boxes: one for CHWs and one for nursing officers. An equal number of names were then randomly drawn from each of the boxes. This procedure was done in both the government and church related health facilities.

Before the study commenced, the selected participants were given the information sheet with the consent form (Appendices 1 and 2). The information sheet was purposively given to each of the participants to read and have a clear understanding of the actual study. It contained the aim of the study which was further explained in detail. In the form they were made aware of the ethical approval for the research that had been given by Victoria University. Moreover, the researcher made sure to explain in the information sheet the channel of communication that was taken for the study and to further justify why some of the approached had not been selected. More explanation on the duration of the interview and their rights to withdraw from the study were outlined. The confidentiality of the research was reinforced before the interviews. Moreover, the researcher's contact details were made known to the volunteers should there be any questions or queries that they would like to raise. After random sampling, the selected participants were informed verbally and the consent forms were distributed. 


\section{Procedure of the participants' involvement}

The selected participants were asked to take part in semi-structured interviews, each of which lasted for approximately 30-45 minutes; this was the main method used for data gathering. The interviews conducted were between late July and early September 2017. The interviews were carried out in Tok Pisin which is the common language across most of PNG. In each interview, the open-ended questions were asked (Appendix 3 ) and the responses were audio-taped. The interview was done according to the participant's availability and the venue was selected by the manager based on the vacant rooms within the health facilities. The managers were cooperative as they chose a room which was less distractive and informed their staff of the venue to avoid causing an inconvenience. Moreover, the participants were made aware of their interview times every morning by the managers and they were reminded again five minutes before their interview. The managers arranged everything because they regarded the research as important and worthwhile.

\section{Handling information}

The raw data extracted was kept confidential. The data was kept on a hard drive, which was accessible only through the use of a password. The recorder was kept in a locked suitcase where all the personal and confidential materials were kept, and it was kept there for the duration of this project. The only person to have access to all this was the researcher and the research supervisor, who had access to the transcripts. Information related to the participants such as the audio tapes has already been destroyed and transcripts will be kept in a secure place until the study is complete and submitted. These records will be held for five years thereafter the completion of the project and then destroyed.

\section{Confidentiality}

It was made known in the information sheet that the identity of each participant was to remain anonymous. Whatever they said that was recorded was kept strictly confidential between the researcher and the participants. They were told that the only person to have access to their data other than the researcher would be the research supervisor. 


\section{Data Analysis}

The raw data as audio-taped was played repeatedly for clear understanding of the participant's view; this was later translated by the researcher from the common language Tok Pisin into English ensuring detailed information was provided at an appropriate level. Because literal translations of Tok Pisin sometimes leads to possible misinterpretations, the transcribed data was typed and checked against the voice recordings repeatedly for clearer understanding and editing. The participants remained anonymous on the transcribed data as they were identified only by their code names on all the transcripts.

Furthermore, the transcribed data was printed out and the hard copy was used for coding. On the hard copy, sentence to sentence open coding was done to code the raw data. Throughout the coding process, the researcher ensured that each sentence was answering the question: "What is the main point?" Each term that was considered to be the main point was underlined and phrases or notes were written besides each sentence on a hard copy. Based on the thematic interpretation of the main points of the sentences, smaller codes were devised and eventually fully named.

These smaller codes were placed in a diagram very similar to Figure 7 to identify their similarities and overlaps. Similar diagrams were drawn repeatedly over time, and the codes were also rearranged and reorganised to develop themes based on similarities. The study ensured that an overarching theme was developed that reflected all the codes in each specific category. Often, these themes were drawn on the whiteboard and were discussed with the supervisor who together with the researcher, considered their relationships, overlaps and accuracy of interpretation.

Themes were further arranged in a table (Table 1) under the participants' code names for further analysis, reorganisation, and refinement of the themes. Similar codes were highlighted with the same colour to identify the codes which occurred the most to the least. It was during this phase where some of the themes identified were decided to be not truly accurate 
representations of themes and everything under those themes was reorganised to become a new theme or to more closely fit another existing theme. Table 1 outlines factors that influences individual nurses' performances as per their views.

Table 1: Initial phase of thematic codes for rural nurses' motivation in Papua New Guinea.

\begin{tabular}{|c|c|c|c|c|c|c|c|}
\hline $\begin{array}{l}\text { Themes/ } \\
\text { Names }\end{array}$ & $\begin{array}{l}\text { Staff } \\
\text { shortage }\end{array}$ & Safety & Income & Culture & Education & $\begin{array}{l}\text { Accomm } \\
\text {-odation }\end{array}$ & Others \\
\hline N1 & $\begin{array}{l}\text { Qualified } \\
\text { Nurses } \\
\text { Recruitment } \\
\text { Manpovwer } \\
\text { Work } \\
\text { Overload } \\
\text { Position }\end{array}$ & $\begin{array}{l}\text { Equipment } \\
\text { Water }\end{array}$ & Salary & 2 & & & \\
\hline N2 & $\begin{array}{l}\text { Qualified } \\
\text { Nurse } \\
\text { Work } \\
\text { Overload }\end{array}$ & $\begin{array}{l}\text { Hospital } \\
\text { upgrade } \\
\text { Equipment }\end{array}$ & & $\checkmark$ & & $\checkmark$ & $\begin{array}{l}\text { Limited } \\
\text { access to } \\
\text { basic } \\
\text { services }\end{array}$ \\
\hline MN1 & & $\begin{array}{l}\text { Hospital } \\
\text { Upgrade } \\
\text { Work } \\
\text { overload } \\
\text { Health } \\
\text { infrastructure } \\
\text { Equipment } \\
\text { Road } \\
\text { infrastructure } \\
\text { \& transport }\end{array}$ & $\begin{array}{l}\text { Salary } \\
\text { Allowance }\end{array}$ & & $\begin{array}{l}\text { Children's } \\
\text { Education }\end{array}$ & 2 & \\
\hline MN2 & $\begin{array}{l}\text { Manpower } \\
\text { Work Over } \\
\text { load }\end{array}$ & $\begin{array}{l}\text { Old health } \\
\text { facility } \\
\text { Equipment } \\
\text { Poor working } \\
\text { condition }\end{array}$ & Salary & $\checkmark$ & $\begin{array}{l}\text { Training } \\
\text { Lack } \\
\text { Knowledge } \\
\text { \& Skills }\end{array}$ & & \\
\hline N3 & $\begin{array}{l}\text { Manpower } \\
\text { Work } \\
\text { overload }\end{array}$ & $\begin{array}{l}\text { Water } \\
\text { Road } \\
\text { Transport }\end{array}$ & $\begin{array}{l}\text { Fund } \\
\text { Salary } \\
\text { Incentives } \\
\text { Allowance } \\
\end{array}$ & & & & $\begin{array}{l}\text { Drugs } \\
\text { shortage }\end{array}$ \\
\hline N4 & $\begin{array}{l}\text { Manpower } \\
\text { Work } \\
\text { overload }\end{array}$ & $\begin{array}{l}\text { Water } \\
\text { Electricity }\end{array}$ & $\begin{array}{l}\text { Fund } \\
\text { Salary } \\
\text { Incentives } \\
\text { Allowance } \\
\end{array}$ & $\checkmark$ & & & $\begin{array}{l}\text { Drugs } \\
\text { shortage }\end{array}$ \\
\hline N5 & $\begin{array}{l}\text { Manpower } \\
\text { Work } \\
\text { Overload } \\
\text { Bias } \\
\text { Qualified } \\
\text { Nurse }\end{array}$ & $\begin{array}{l}\text { Health } \\
\text { infrastructures }\end{array}$ & $\begin{array}{l}\text { Salary } \\
\text { Overtime } \\
\text { Pay } \\
\text { Incentives } \\
\text { Rec. Leave } \\
\text { Pay }\end{array}$ & $\checkmark$ & Study & 2 & \\
\hline N6 & $\begin{array}{l}\text { Bias } \\
\text { Manpower }\end{array}$ & $\begin{array}{l}\text { Health } \\
\text { Infrastructure } \\
\text { Water } \\
\text { Safety }\end{array}$ & $\begin{array}{l}\text { Salary } \\
\text { Overtime } \\
\text { Pay } \\
\text { Incentives } \\
\text { Rec. Leave } \\
\text { Pay }\end{array}$ & Beliefs & Study & 2 & \\
\hline N7 & $\begin{array}{l}\text { Workload } \\
\text { Manpower }\end{array}$ & $\begin{array}{l}\text { Health } \\
\text { Equipment } \\
\text { Water }\end{array}$ & $\begin{array}{l}\text { Salary } \\
\text { Overtime } \\
\text { Pay } \\
\text { Incentives } \\
\text { Rec. Leave } \\
\text { Pay }\end{array}$ & $\checkmark$ & $\begin{array}{l}\text { Children's } \\
\text { education } \\
\text { Training }\end{array}$ & & \\
\hline MN3 & & $\begin{array}{l}\text { Health } \\
\text { infrastructure } \\
\text { Road } \\
\text { Infrastructure } \\
\text { Health } \\
\text { equipment } \\
\end{array}$ & Salary & $\checkmark$ & & & $\begin{array}{l}\text { Hospital } \\
\text { structure }\end{array}$ \\
\hline
\end{tabular}

Some of the themes listed in Table 1 were again refined, renamed, and rearranged. Different sub-themes (Figure 9) were developed from the codes, ensuring the sub-themes were related to the main topics. 
Factors that affects nurses' motivation in rural areas of Papua New Guinea

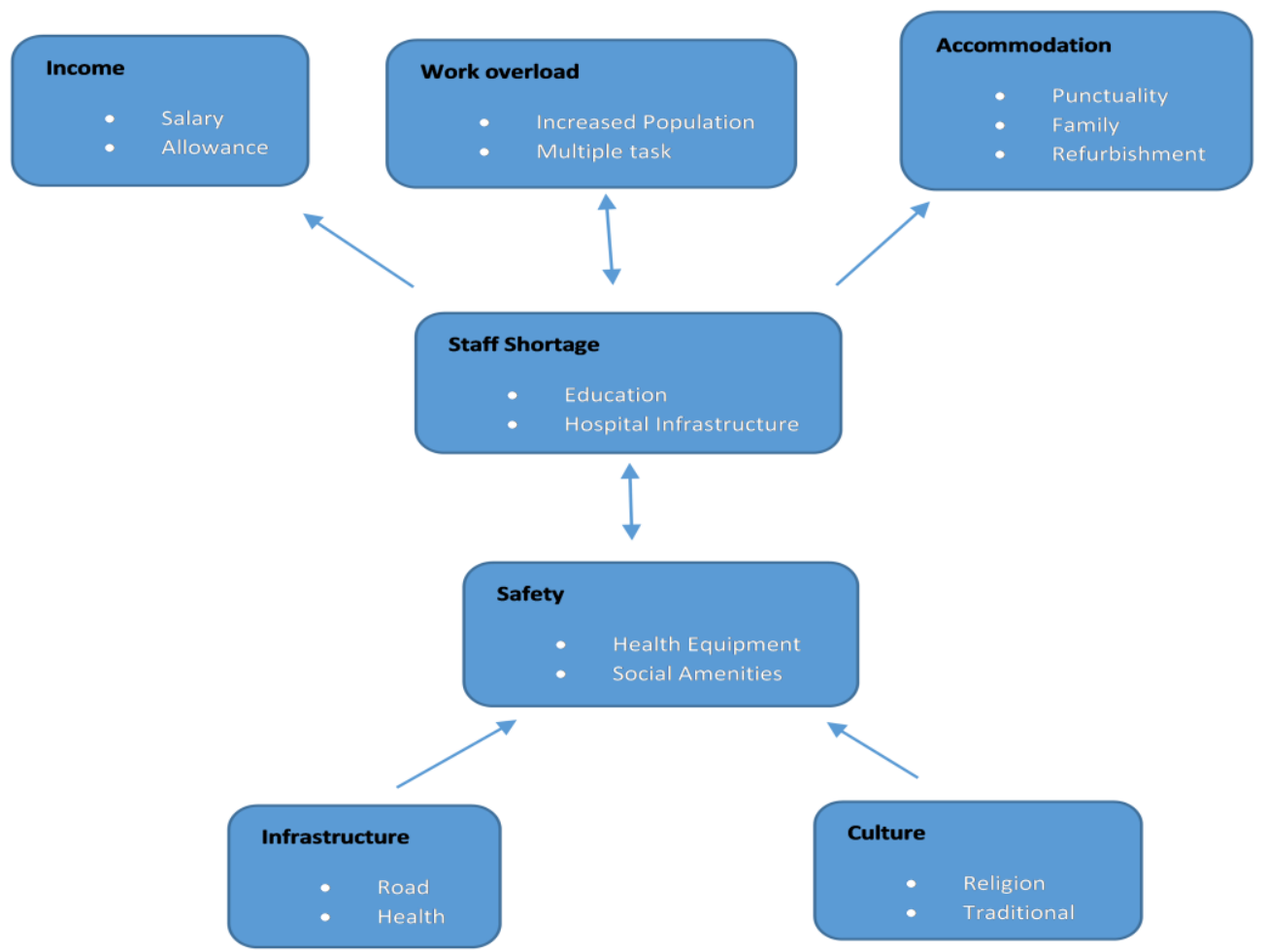

Figure 9. Final themes for nurses' motivation in rural areas of Papua New Guinea.

Seven main themes with their sub-themes were developed after many processes of rearranging, refining, and changing the names of themes and sub-themes. These seven themes stood out to be the main outcomes of the data despite exploring repeatedly for any chances of creating new themes or modifying existing ones. Based on this arrangement of themes, a model (Figure 10, please see next chapter) was developed which outlined both codes and themes within a suitable thematic framework.

\section{Distribution of the Results}

A copy of this thesis will be issued to:

- The health facilities that participated so that nursing staff can have access to it

- The Graduate School of Nursing, Midwifery and Health at Victoria University of Wellington

- The Provincial Health Authority, PNG 
- $\quad$ The PNG Health Department

\section{Summary}

Lacking nurses in rural areas of PNG is one of the major issues that is currently faced by the country. However, with only two available examples from the literature, the arguments are weak when attempting to further identify the causes of problems in this area of nursing practice. As such, this research was conducted with 10 practising nurses in rural health centres in PNG to investigate further and to strengthen some of the arguments that have been highlighted by another two studies. With the main focus to explore and identify the causes of nurse numbers declining in rural areas, a descriptive-exploratory approach was employed with the use of thematic analysis to analyse its data using an inductive approach. Based on the data, seven main themes were identified; income, work overload, accommodation, staff shortage, safety, infrastructure, and culture which will be discussed in the next chapter. It is hoped that this research will have an impact on the health policy planning and resourcing at national, provincial, and local levels. 


\section{Chapter Four: Findings}

\section{Introduction}

This chapter presents an analysis of data collected from the interviews with the selected participants. The focus of this chapter is to present the analysis of the motivating and demotivating factors that enable rural nurses in Papua New Guinea (PNG) to continue working under difficult conditions. The main reason for this analysis was to investigate the main problem of rural nursing staff shortages and how rural nurses dealt with this within the PNG context. Data analysis revealed that safety and socioeconomic factors were the main issues in the nursing shortages in the PNG context. Based on this analysis, six sub-themes were developed which were categorised under two main themes, namely safety and socioeconomic factors (as listed in Figure 10).

The themes based on safety were found to largely concentrate on workplace safety, while the analysis of socioeconomic factors revealed further themes relating to the social and economic aspects. Safety comprised of the sub-themes basic amenities, health services infrastructure and human resources. Whilst socioeconomic factors were best explained by the sub-themes; income, culture, and accommodation issues.

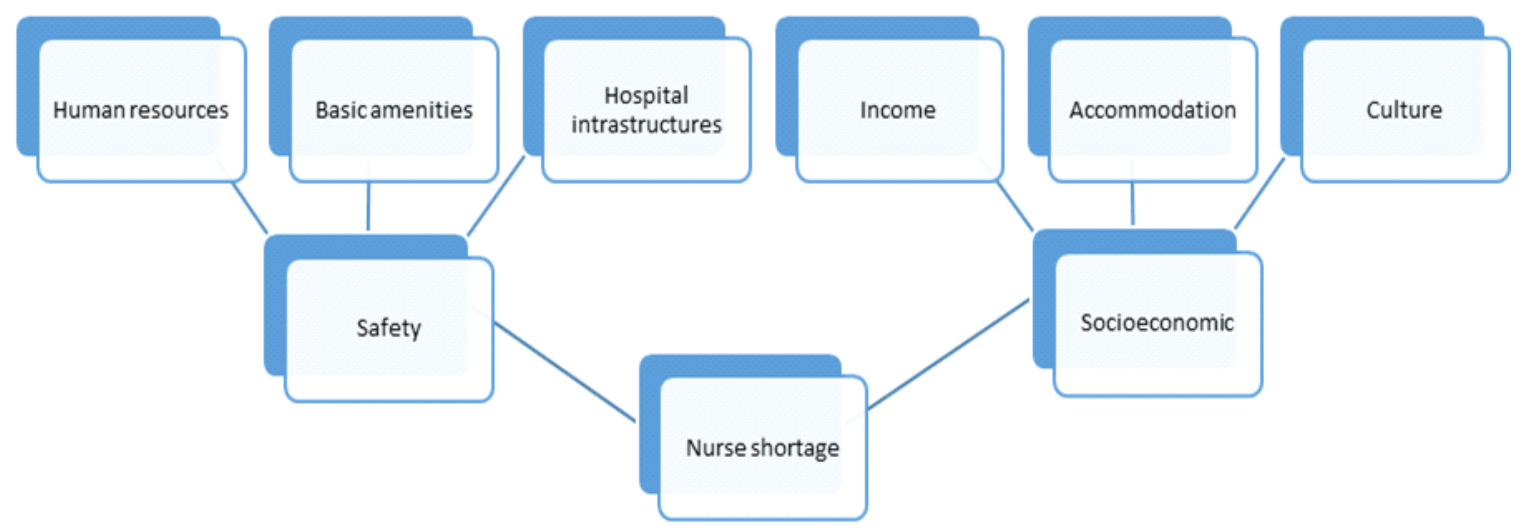

Figure 10. Motivational factors for rural nurses in Papua New Guinea. 
Before an analysis of the personal and workplace safety is explored, the chapter will firstly look at the participants' job histories, the make-up and performance of rural nurses, and compare this to their realities in the workplace through an analysis of the interview data.

\section{Participants' Histories}

Table 2: Description of participants' histories.

\begin{tabular}{|llllllll}
\hline $\begin{array}{l}\text { Description/ } \\
\text { Nurses }\end{array}$ & Gender & Organisation & Title & Code & $\begin{array}{l}\text { Years of } \\
\text { service }\end{array}$ & $\begin{array}{l}\text { Rural } \\
\text { experience }\end{array}$ & $\begin{array}{l}\text { Urban } \\
\text { experience }\end{array}$ \\
\hline 1 & Female & Government & CHW & N1 & 32 & 30 & 2 \\
\hline 2 & Female & Government & RN & N2 & 12 & 10 & 2 \\
\hline 3 & Male & Government & CHW & MN1 & 42 & 42 & Nil \\
\hline 4 & Male & Government & RN & MN2 & 27 & 26 & 1 \\
\hline 5 & Female & Church & RN & N3 & 17 & 9.5 & 7.5 \\
\hline 6 & Female & Church & CHW & N4 & $20+$ & $20+$ & Yes \\
\hline 7 & Female & Church & CHW & N5 & 7 & 7 & Nil \\
\hline 8 & Female & Government & CHW & N6 & 27 & 27 & Nil \\
\hline 9 & Female & Government & CHW & N7 & $7+$ & $7+$ & Nil \\
\hline 10 & Male & Government & RN & MN3 & $35+$ & Yes & Yes \\
\hline
\end{tabular}

Key: CHW-Community Health Worker: RN - Registered Nurse:

From the data shown in Table 2, it is obvious that the list of participants lacks complete balance in regard to gender, organisation, and title. For clarity, the study sites are female dominated with more nurses in the government related health facilities than those run by the church. Also, the $\mathrm{CHWs}$ comprise a large part of the population of rural nurses. It was impossible to maintain equality in recruiting participants in terms of organisation, nurses' occupations, and gender especially when participants' confidentiality is the priority in the research. Therefore, this study has applied an appropriate approach to select adequate participants with priority to their confidentiality. Hence, the data shown above is the suitable selection. Moreover, the participants were given code names; N1-N7 refer to female participants while MN1-MN3 refer to the males. Their arrangement in Tables 2 is in the order of the interviews.

Furthermore, Table 2 shows that all these nurses have at least seven years of work experience. Over $60 \%$ of the participants have more than 10 years of rural experience. Of these, only one had worked only in the post-independence period and is still practising, while the rest were 
recruited pre-independence (hence many of the participants had extensive experiences of both major systems of health delivery in PNG - as previously described in Chapter 1). All of the participants recruited had sufficient experience of rural practice. Indeed, two nurses have 29-45 years of services, another three nurses had 20-30 years of service, another three nurses had 1019 years and one nurse had at least seven years of service.

\section{An Overview of Nursing Responsibilities in the Rural Areas of Papua New Guinea}

Overall, nurses in the rural areas of PNG play absolutely vital roles in health service delivery, and in the absence of doctors and other health workers (such as Health Extension Officers), these nurses have to cover most of the health delivery requirements. As a result, analysis of the interview data quickly revealed that all rural nurses in PNG frequently go above and beyond their core roles. One participant stated that 'In rural practice, we do everything from admissions to discharge' (MN1). This was further elaborated by his colleague who explained that 'I perform multiple tasks beyond my scope apart from my nursing roles, I work as a health extension officer and a doctor by performing examination, admission, treatment order and insert intravenous cannulas for patients' (N1). What this shows is that while rural nurses understand their limitations they are quite often challenged in their role to perform extra functions.

The PNG concept of 'wantok' (as described in Chapter 1) is normal for the community and is one of the examples of the pressures on rural nurses to perform these extra functions. This is where nurses voluntarily commit their time to wantok whether inside or outside of their duty hours to deliver health care. The wantok system literally demands that anyone apart from relatives whom the nurses know such as friends, teammates and neighbours must receive cooperation and assistance as required. It is the norm in PNG to commit time to help a wantok in any organisation and not only in health matters. As stated, the nurses often work for the love of their job and to serve their community; this is especially the case where the wantok system is operating. 
Subsequently, nurses who live in communities closer to the rural health facilities often volunteer to help on their day off. N8 explained that 'l come in anytime during my off days to help whenever [I] am needed because nurses are limited: I help voluntarily for humanity'. On a similar note, another participant (MN1) mentioned that 'I travel to remote communities to deliver health services when needed'. What this means is that nurses are challenged between work safety and community obligations. In this instance, nurses that go into the isolated communities are serving two purposes; serving their community and preventing health related ailments (such as tuberculosis, leprosy, and measles) from escalating to bigger health issues (which might threaten the lives of the local community).

On the same note, rural nurses ensure that everyone who visits the facilities are served as well as is possible. Despite having limited staff, nurses attempt to cater for all those who visit their health facilities, ensuring that each individual receives adequate care. This is mentioned by participant N6 who pointed out that: 'We have a large number of patients compared to staff... we normally finish late in the afternoon with no lunch break'. Furthermore, N2 added that 'when there is less work in my section, I often go across to the outpatients, particularly the antenatal clinic, and assist other nurses by attending to mothers and their babies, including paediatrics'. Such comments strongly imply that rural nurses are versatile within their respective health facilities to provide health care to the demanding population and ensure everyone is served to the best of their abilities.

Gathering from the participants' responses, and including the concept of wantok, rural nurses appear to have more empathy with their patients than urban nurses. Thus, rural nurses often exhibit considerable sympathy for their patients in the rural community, and they do everything they can to support them. Indeed, although they are vastly outnumbered by the patients on a daily basis, rural nurses generally try to ensure that everyone is served by working into their personal time to deliver health care. Again, this reinforces the notion that they are often overstretching themselves in order to serve their community, and in many respects, this affects various aspects of their overall safety. 


\section{Safety}

Safety is one of the overarching themes to emerge from data analysis, meaning nurses are more concerned with the 'internal factor' of safety than many other factors. The study reveals that safety at the work place is highly prized by rural nurses because without it, they cannot achieve their aims. At the current stage, many rural health facilities in PNG are unsafe for the nurses, however the nurses still practice despite these conditions to serve their communities and prevent further illnesses. They are unsafe for both nurses and patients alike for many reasons, but the participants largely stressed the importance of a safe working environment and the impact it will have on their practice. For clarity, safety is not particularly about personal safety (as in being physically attacked) because almost all of the nurses in this study are from the surrounding villages with relatives and friends around. However, safety here applies to three main sub-themes namely human resources, basic amenities, and health services infrastructure. These all play a vital role in the delivery of quality health services which have a significant impact on workers' practices.

From the overview in Chapter 1, it is known that rural locations in PNG contain over $85 \%$ of the total population. The rural population growth has increased dramatically over the past years with resulting problems of PNG villagers facing food shortages and other major problems (Tlozek, 2016). Consequently, the limited numbers of nurses are overwhelmed with other responsibilities apart from their nursing jobs (Figure 11). As previously stated in this study, and as reinforced by participant N2 'the majority of the population lives in rural locations'. As such, even basic requirements such as an adequate food supply, accommodation, and water and electricity supplies have become significant health and safety issues. However, the recruitment of staff to rural locations is too limited to cater for the growing population and their needs. This was further elaborated by N6 who explained that:

We have more isolated villages that surrounds this small health centre and we overwork ourselves. We work during the day and continue serving through the evenings and nights, we have sleepless nights when we have more cases of 
emergency and we serve continuously to the next morning shifts...we critically need more staff to help lighten the burden.

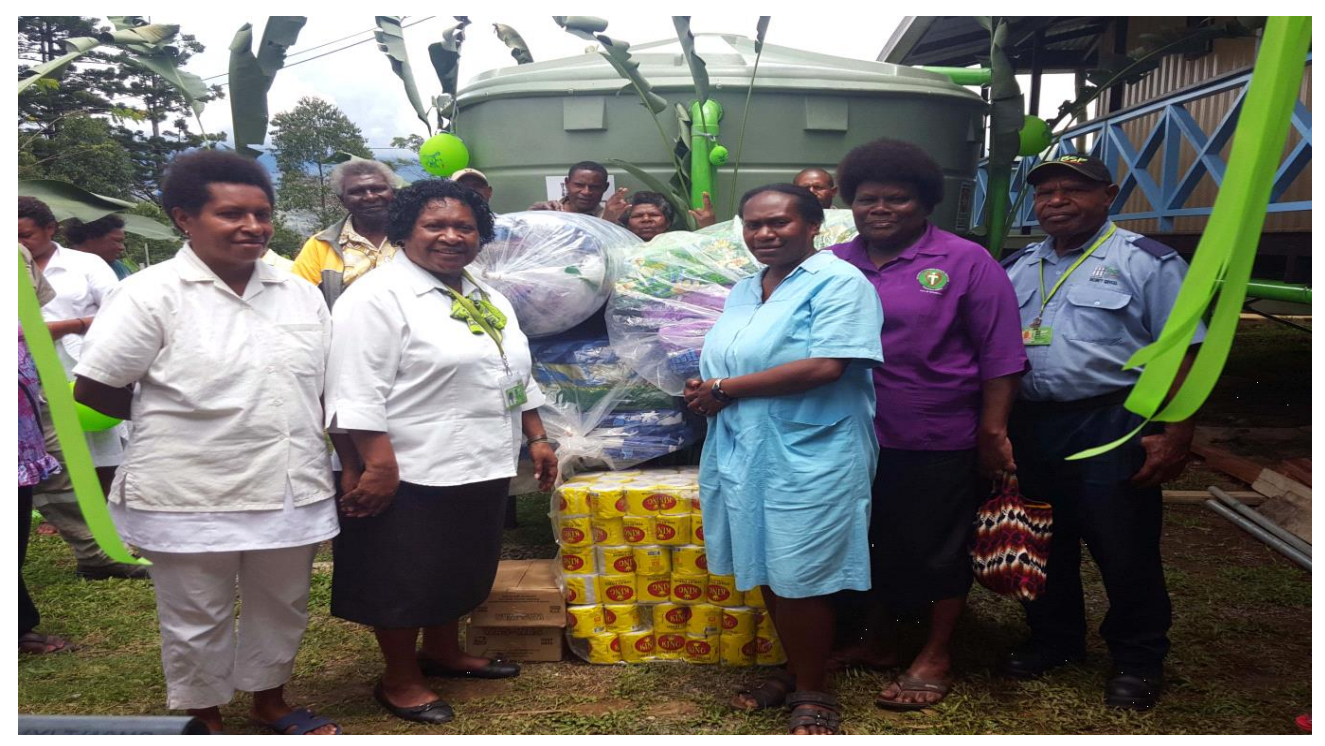

Figure 11. Rural health facilities receiving donations from NGOs

\section{Human resources}

A lack of human resources in health is common in all rural areas of PNG. As a result, rural nurses do a variety of jobs to maintain the function of the health facilities despite the increasing population in which they are challenged by work safety and community obligations against continuous population growth and demands. The population increase has clearly added extra burdens to limited nurses, as MN1 indicated:

In the past the health facilities expected less patients because there were more aid post[s] in remote and other rural locations with less people. However, we are now receiving more patients because all the aid posts have been closed including some of the rural health facilities, but the population had increased and people are flooding in every day.

Similarly, N3 concurred that 'the rural and remotest part[s] of the districts had health centres or aid posts during pre-independence but most of these do not exist today, and as a result we see more patients'. As noted by the participants, rural health facilities are much busier in post- 
independence than pre-independence times which had the effect of having less health facilities and shortages of health professionals.

Another participant (N2) added that "I have served urban locations but their health facilities are not as busy as rural health centres.' This statement implies that the existing rural health centres are much busier now than in earlier days and are extremely busier than urban hospitals at the current stage. This is because the increase in health centres is not on par with population growth. Instead, it appears that the increasing of the rural population has actually been mirrored by a decrease of health facilities and has added extra work for the limited nurses. Thus, nurses overwork themselves to maintain the delivery of health services, and frequently find this difficult. Having more health facilities in the past decreased the workload compared to the present with less health facilities and an expanding population.

However, rural nurses are versatile, and many change easily from one job in the health centre to another to maintain the efficient delivery of health care. One of the participants N1 reported that 'nurses in the ward does the cleaning, scrubbing and mopping, provide nursing care and furthermore suture and insert intravenous cannulas'. This indicates that they oversee the work of rural health facilities by voluntarily taking on different tasks outside their practice environment, some of which are actually below their level of competency (e.g. cleaning and mopping), and some which are clearly above (e.g. inserting IVs and suture work) without special training. On the same note, N2 elaborated on her work routine by explaining that she often worked in areas beyond her regular work unit by explaining that: 'I do the rounds for Gynaecology cases, administer their treatment, assist nurses in paediatrics clinics and return to the labour ward'. Hence, although stationed in their respective wards or units, nurses help each other by moving around between different areas within the health centre; i.e. they move to different sections where help is needed and assist their colleagues to reduce the work load.

In the above scenarios, nurses are significantly challenged. However, this model is not sustainable as it impacts personal and workplace safety, i.e. nurses are caught in 'catch-22' 
situations. In this, rural nurses are not confined just to their appointed tasks; instead they work together as a team across all areas for the betterment of people and offer a variety of services that would normally be provided by a range of other health workers. This is because although rural nurses lack some specialist skills, the needs of the increased population have forced them to expand their practice. As a result, their continuous exposure to different procedures has broadened their knowledge and they are more competent at performing certain procedures outside and beyond their scope of practice. This however, comes at a price inasmuch as such work may often lead to exhaustion and possible mistakes. Yet, due to population increases, rural nurses have to place greater emphasis on team work to address the needs of the majority. However, the issue of nurse shortages will still exist unless the hospital and health centre structures and recruitment of nurses within them is reformed.

\section{The recruitment of nurses}

The recruitment process of the nurses is done by the Human Resources department within the PHA and not locally. The recruitment is dependent on the hospital structure rather than a community model which does not reflect a rural community (Kolehmainen-Aitken, 1992). The structure of the hospital varies but overall it is grounded on bureaucratic and hierarchical processes. The hospital structure contains the information on different career opportunities for nurses in the rural health facilities. This includes positions such as general nurses, midwifery, paediatric nurses and so on within the health facility. This means that the hospital structure needs to be updated frequently to meet the demand of the rural population by including other positions of the newly established sections. However, what this means is the model they use for recruiting in the urban areas is not suitable for rural health.

The issue of nurse shortages in rural health facilities is significantly different from other urban health facilities. As such, the hospital structure must be framed differently from the urban structure to meet the needs of rural health. One of the participants stated that 'we have been using the old structure for many years' (MN2). Hence, even though the old structure was not explicitly defined, it is more likely referring to the structure that was used in pre-independence 
when the health system was centralised. Subsequently, a lack of adjustments and modifications to the new structure has greatly affected the recruitment of staff, therefore creating an imbalance between urban and rural nurses, with implications for safety when the numbers in rural areas are insufficient to do the work.

This all implies that the rural health facilities in PNG need a model of their own, centred on the decentralised system, to recruit nurses. Rural health needs a new hospital structure more focused on preventative health. However, as one participant mentioned:

The delay in the hospital structure has been going on for ages, the provincial health authority has failed us in this by not speeding up with the new structure... we have waited so long for that and most of us had already reached our retirement age. (MN2)

Another participant supported this notion by pointing out that 'the shortage of nurses is caused by [the] PHA because of their never-ending delay in hospital structure' (N4). It follows that some nurses working in rural health settings in PNG obviously perceive the problem of adequate staffing to be a managerial issue at provincial levels. Lacking a new hospital structure and model therefore most likely affects the nurses' recruitment to rural locations. However, the PHA has been slow with updating and changing the structure. Thus, the rural health facilities still use the old hospital structure which has disabled the recruitment of adequate nurses to meet these demands. The delay of the necessary structural improvements is, in part at least, associated with poor communication.

\section{Communication issues}

Poor communication among different levels of the health services is a major issue affecting most of the health delivery systems in PNG. As participant MN1 stated, 'the government critically need to do something about this hospital as the population had increased and we face difficulties in delivering services'. Such difficulties mean that the service becomes increasingly unsafe; yet the government will not help unless the issues are raised and brought to their 
attention through effective communication. It may be argued that the two layers of a reporting system which was introduced by the decentralised system disabled the effective communication from pre-independence times onwards. There is a lack of communication and the NDoH lacks information on some of the issues that are faced by the rural areas (Asian Development Bank, 2015). Lacking information has a direct impact on distribution of funds and development of health policies to address specific rural needs.

On a related matter, participant MN2 concurred that 'the government needs to look into some of the health issues'. As such, health workers are calling on the government to address the issues, but again it needs effective communication for the problem to be solved. Yet the problem is not only a government run institutional issue, for instance, a nurse and hospital manager (N5) in a church related health facility explained that 'over 50\% of our salary was previously deducted by the church which is too much. We complained and as I was the hospital manager we brought this to the attention of the CEO and it was sorted out'. In this instance, i.e. in a church related health facility, the channel of communication was effective, the subordinates brought their complaints to the manager which was relayed to the CEO and was addressed immediately. Applying such an approach effectively within the three levels of government which are local, provincial, and national is therefore a possible solution to addressing this particular aspect of rural health.

As mentioned, sustaining nurses in rural areas is achievable but not at the current rate, and with weak communication between provincial and local health. Nurses prefer practising in a well-managed, well-staffed, and safe environment to provide preventative and curative care. However, these needs are yet to be achieved due to poor communication among PHA and DHA. Thus, nurses struggle with limited resources (such as lack of adequate amenities) to deliver health care. 


\section{Basic amenities}

It became apparent during the analysis that lacking basic social amenities such as water, electricity, and medical supplies was a major issue in the retention and job satisfaction of rural nurses. These issues open the door for unsafe conditions and practices by exposing the nurses to a variety of safety hazards. Working under these conditions is life-threatening for the patients as well as the workers which is a huge challenge for their well-being and hygiene needs. Although water and electricity are vital in all health practices, most rural health facilities are affected by significant water and power shortages.

\section{Water and power problems}

In tropical climates such as PNG, water is very important because it acts as the primary defence to prevent cross infection. One of the participants (N5) explained that 'we are faced with the worst problem of lacking a proper water system' (Figure 12). Further to that, N6 elaborated that '[the] poor water system is one of the main causes of increased cases of typhoid which has caused more deaths...I am currently diagnosed with typhoid because of this water issue'. Subsequently, water issues have negative impacts on the health of both the staff and patients.

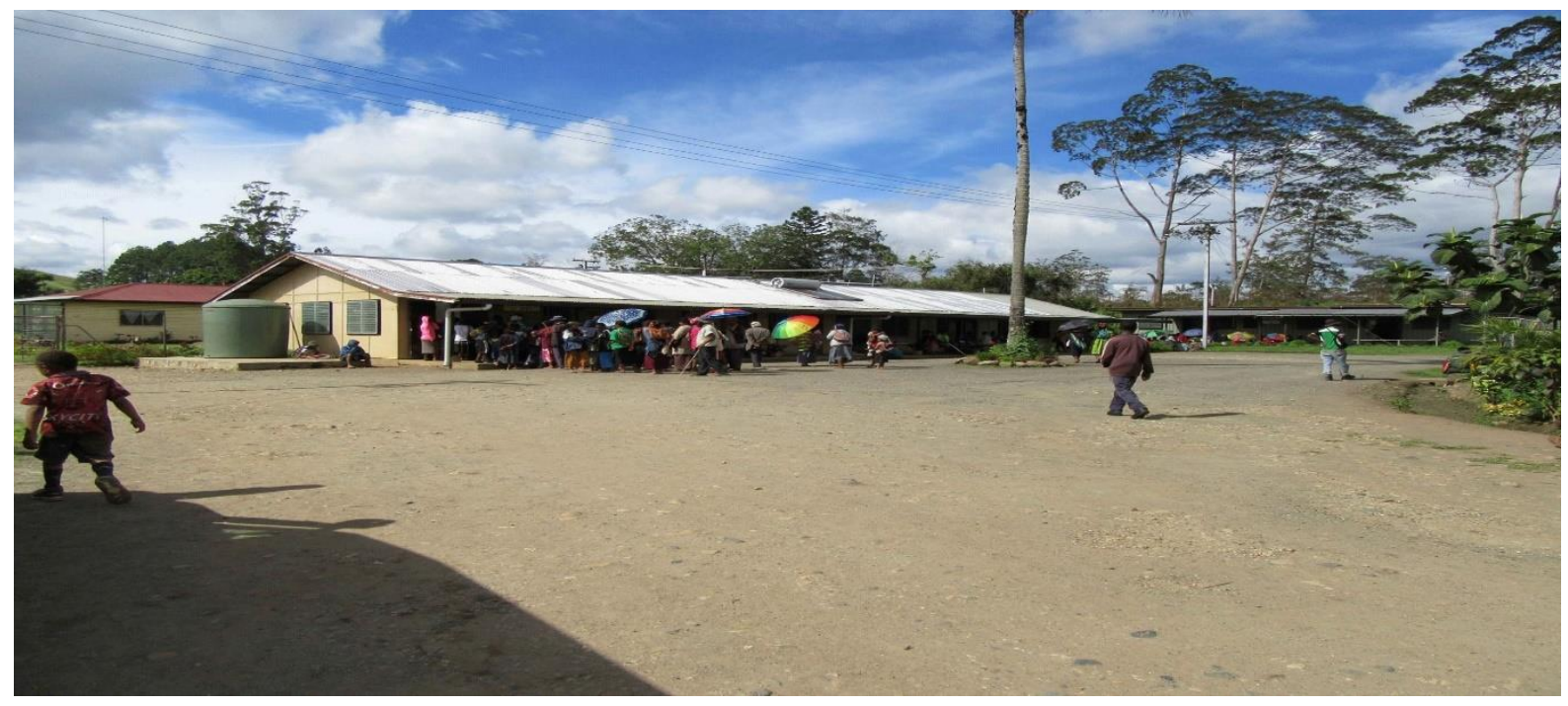

Figure 12. Rural hospital using tank water for health care. 
Several participants noted issues with water supplies. N1 stated that 'we use tank water, which is unhygienic'. Moreover, N7 concurred that '[the] shortage of water has [a] negative impact on health service delivery'. Tank water which is stagnant is usually contaminated and accumulates dirt that contains a variety of unseen bacteria. As noted by participants, the majority of the rural areas are reliant on tank water which affects their health. Communicable diseases such as typhoid and diarrhoea are very common in rural areas of PNG. As such, a lack of water enables the fast spread of contagious diseases which affect both the staff and patients. This distresses rural health nurses such as the participants of this study due to the unnecessary health problems that are caused by a poor water system at their health centres. In saying this, another contributing factor to the spread of bacteria and other health problems is the unreliability of the electricity supply.

A reliable source of electricity is a challenge for nurses to the delivery of adequate health care. Quite often while in the community they carry on delivering health services regardless but it makes everything much more difficult. N5 reported that 'electricity is vital in rural areas because we attend to similar cases as during the day and it is very difficult to work without electricity in the night'. Moreover, N6 explained that 'I work in dim light in emergency cases such as deliveries'. Further, MN2 added that 'we risk our lives working in such an environment where it is known for having high rates of HIV and TB cases'. Most of the participants therefore noted that unreliable electricity is unsafe and detrimental to their work. However, they continue serving their community. Yet, there are other major challenges, and one of the most pressing is that of unsafe practices associated with the use of pharmaceuticals.

\section{Drug shortages}

Drugs play a vital part in the recovery of patients especially in isolated locations where access to further medical support such as operations or follow-up is difficult. MN2 clarified that 'other health centres who lack drugs refer patients to us'. As such, 'this rural health facility serves more districts than expected' (N9). As the districts health facilities serve beyond what is normally required, N5 clarified that 'we are faced with the foremost issue of drug shortage which 
impedes the health care delivery'. Consequently, MN3 concluded that 'the health facilities are shut down frequently until new stock arrives from [a] provincial base hospital'. To prevent the shutting of the health facilities, participants N6 stated that 'we try our best to reduce administered drugs while waiting for new stock'. Such an approach, even though it probably saves most lives in critical periods, simultaneously harms other patients as quite often they are under dosed and at risk of a deterioration in their health status. In many cases, and especially in a region where infectious diseases are prominent, taking a lower dose than required enables the bacteria to be resistance to drugs, which is life-threatening to patients in the future in regard to medical help. As a result, nurses in rural areas are often distressed by the lack of sufficient pharmaceutical support and the negative effects on the local population's (including themselves) safety from diseases, especially infectious ones. When combined with another important issue, namely inadequate equipment, the results are often highly distressing for the nurses

\section{Shortages of medical equipment}

Based on the participants' observations, almost all rural health facilities in PNG lack adequate medical equipment. The lack of basic supplies was a common issue raised as an obstacle in delivering quality services by several participants. For instance, MN2 reported that 'we are in critical need of medical equipment'. MN1 pointed out that such occurs because 'most of the medical equipment is outdated'. As such N1 mentioned that 'I find it very challenging to deliver quality health services due to a lack of vital equipment such as blood pressure machine, thermometer, otoscope, normal saline, urinary catheter and oxygen connecters'. The health equipment required are basics and are very useful in rural locations. Hence, although this may seem to be a minor issue to some, it saves the lives of the majority therein overlooking this is clearly a concern for the participants as rural health professionals.

Apart from the examination equipment, the rural health facilities critically need health care furniture, N2 reported on the labour ward perspective and explained that there are no new beds for patients, the available beds are worn out and tied with ropes to keep them firm. 
Mothers deliver on the floor; we are in critical need of delivery trays, the four trays we have are sterilised after every use'. In regards to sterile equipment participant N9 who does most of the sterilising clarified and stated that 'all used instruments are sterilised in bleach and reused because [the] most important machines such as the sterilising machine has malfunctioned'. According to the nurses, they continue delivering health care with inadequate equipment and though they will treat the existing disease, the patients might leave with another which means that lack of equipment is another safety issue that must be critically addressed.

The lack of basic amenities plays a vital role in health service delivery. Whilst having limited nurses seems to be a major issue, the lack of medical equipment is one of the unforeseen issues that worsens the situation considerably. Poor medical equipment encourages unsafe practice and creates unconducive working environments. Besides medical equipment, a lack of social amenities and basic health services infrastructure negatively affects the working environment.

\section{Health Services infrastructure}

\section{Hospital infrastructure}

In addition to inadequate basic amenities, poor health services infrastructure is another significant safety concern for nurses. Nurses prefer working in a productive environment where their safety, and the safety of their patients is guaranteed. However, most of the rural areas lack proper infrastructure such as adequate buildings and transport which impedes the delivery of health services (Figure 13).

Having proper building infrastructure (such as an adequate hospital or health centre) is vital for the safety of both the patients and staff. However, many participants reported that their buildings were unsuitable because, for instance, 'our health facility is run down' (N9), and 'this facility was built in colonial days' (MN1). In the latter case, this means that the hospital is over 43 years old with no refurbishment since. Furthermore, MN2 elaborated that:

The poor health facility is one of the common demotivating factor[s] to my job performance, the health facility is very old with [a] decaying roof...our theatre is 
very bad... when emergency cases arrive we have no choice but to bring them over to that unsafe area and treat them. As a result, we often have complications such as spread of infection and wound breakdown.

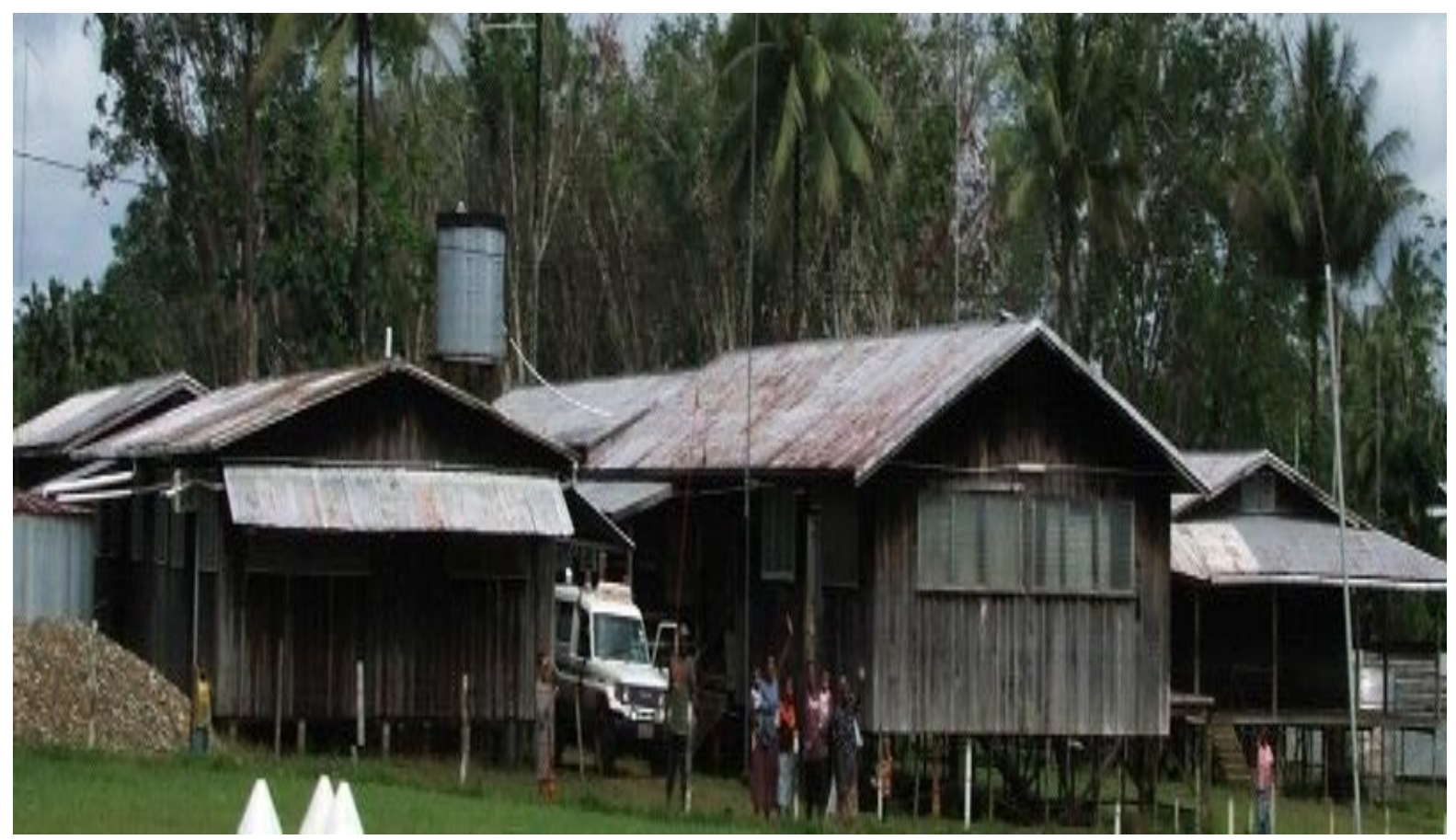

Figure 13. Poor hospital buildings in one of Papua New Guinea rural locations.

This shows that poor infrastructure encourages unsafe practice and is a hazard to both the patients and health workers. Nurses are often challenged in such a way, however they still help to prevent further health issues, whether out of duty or loyalty to their patients, regardless of the poor infrastructure.

The non-existence of some important wards and often a theatre is a concern to most nurses because although the 'majority of the population are served in this health facility' (MN2), sufficient buildings to achieve this are scarce. As one participant noted: 'We have [only] two admission wards, one for paediatrics and [one] other for adults' (MN1). On the same note, another participant from another health facility pointed out that 'we have only one admission ward catering for both the children and adult which is unsafe for both because we are 
promoting cross infection' (N6). To prevent this, N1 suggested that 'the hospital must be extended with different wards to cater for the population'. Overall, the participants indicated that they prefer having a safe practice environment meaning proper safety protocols for nurses must be in place to promote safety. Having safety protocols will enable a safe environment in different aspects such as, creating bigger spaces and building separate wards for certain cases which will reduce the spread of infections. Subsequently, the lack of adequate ongoing renovations has led to the deteriorating of hospital facilities which has in turn created an unsafe working environment for both the patient and staff. Moreover, it has affected the health of the patients who present with one illness but sometimes leave with another.

\section{Road and Transport infrastructure}

In addition to problems with hospital infrastructure, the road and transport infrastructure was also noted as one of the most pressing issues facing several of the participants. Therein a lack of transport has hampered the delivery of health care services in rural locations (Figure 14). As participants N7 reported: 'We find it very difficult to get basic health needs such as drugs due to poor road and transport conditions'. Furthermore, another participant concurred when she claimed that:

Poor road infrastructure is [a] barrier to receive immediate attention from doctors at the provincial hospital and also it is one of the main contributing factors to the frequent closure of this health facility due to [the] inconsistence [of] pharmaceutical drugs supply and drug shortages. (N5) 


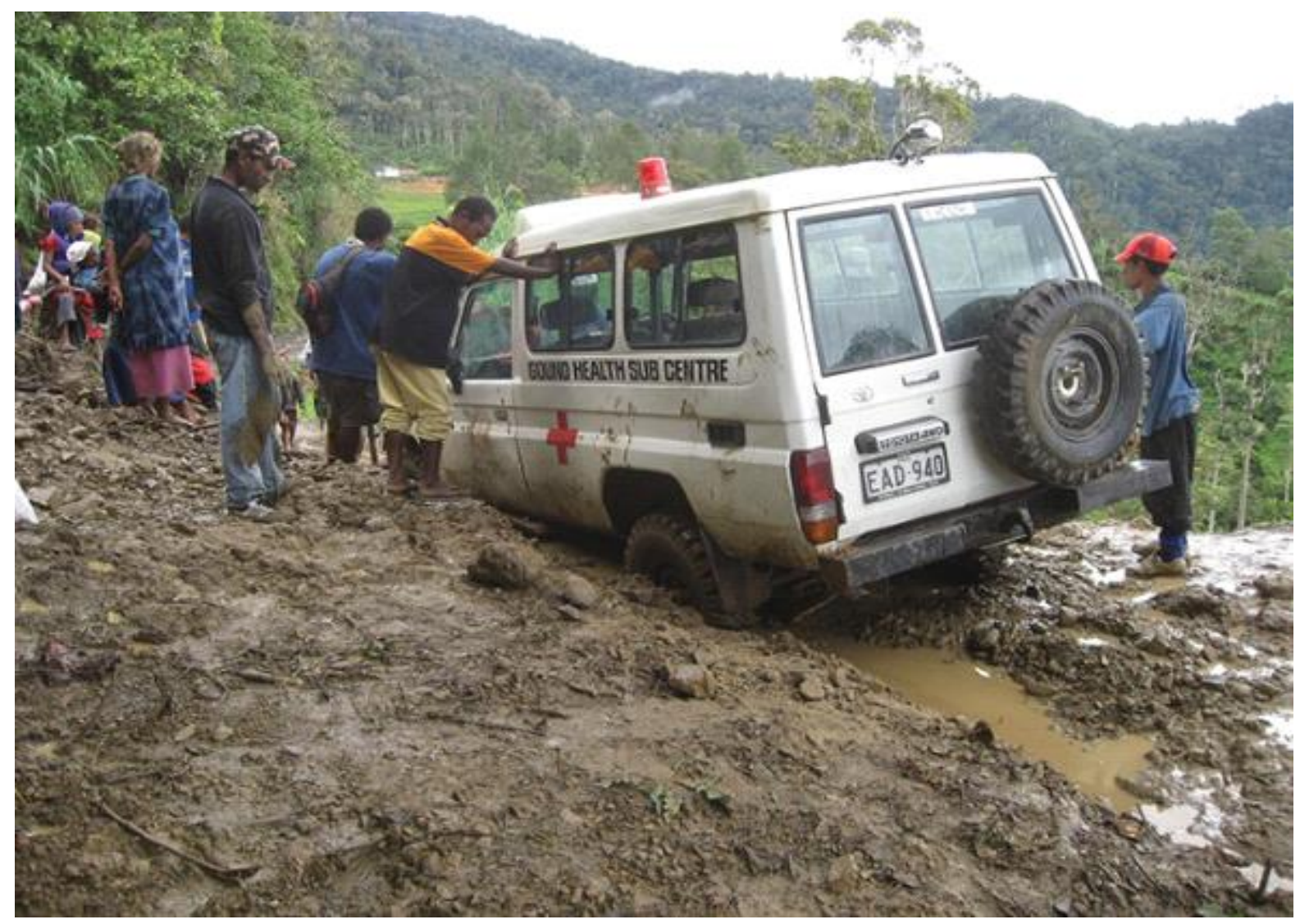

Figure 14. Ambulance transporting sick people from the remote areas to rural hospital stuck in the mud

The participants explained that a lack of proper infrastructure in rural areas impedes the quality delivery of health services and even leads to closure. Hence, rural areas are in crucial need of building infrastructures to cater for the demanding population, but they also need transport to deliver hospital supplies and to transport referral patients to urban health facilities. Several participants indicated that getting patients to the health centre is as much about having adequate medical supplies and roads as it is about anything else.

A lack of transportation to the hospitals makes the situation much worse. Most of the rural health facilities lack ambulances and the people who need special attention are treated at rural centres where there are no doctors and little chance of transporting into a larger area. As one participant explained: 
Lifestyle diseases are increasing annually and we find it very difficult to make final diagnosis because such cases need special attention from specialised people which we lack. Often, we provide systematic treatment to such patients but I feel that we are not really helping and we need to do more. Unfortunately, we cannot do much to such patients because most nurses practising here are general nurse practitioners with less skills to provide special care to such cases. (M1)

However, there is sometimes a transport option for a few seriously ill patients, as MN3 noted when he stated that 'we sometimes get the critically ill patients to the provincial hospital on a church plane'. This form of transport depends on the availability of a service that is often operated by religious-based institutions. Planes are used because in places the road and transport are at their worst. However, it is very costly and people do not use that service very often.

To conclude this section, safety is the main issue identified by the participants. Safety in this study was grounded on workplace safety for both nurses and their patients. The study revealed three main safety issues that were identified by the participants, namely problems associated with human resources, basic amenities, and infrastructures. It has been shown that the lack of health professionals in rural areas has a negative impact on the workers' roles. Limited numbers of nurses means more work and nurses feel obliged to expand and extend their practices with limited knowledge to provide health care. Yet although nurses are eager to give the best, lack of basic amenities are major risks to their health and the health of others. Indeed, some of them reported being the victims of life-threatening diseases due to poor basic amenities. Nurses expose themselves to such risks as a result of insufficient basic amenities. This is not only due to a lack of basic amenities but is also due to a lack of proper health infrastructures such as a proper number of carefully designed and constructed buildings. Also, the lack of infrastructure such as transport also affects the delivery of hospital needs and transportation of urgent specialist cases; it also tends to prevent other health workers from even getting to the health centre in the first instance to assist. Addressing all these issues appropriately will contribute to 
the safe practice environment which will reduce demotivation and increase the motivational factors of nurses to go to, or remain working at, rural health facilities in PNG.

\section{Socioeconomic Lifestyle}

The second main theme in this analysis is socioeconomic status which includes social and economic issues that nurses are faced with in the workplace. This theme is comprised of three main sub-themes; income, culture, and accommodation. Unlike safety, these factors are more focused on the external issues that may be attributed to rural nurses' motivation or demotivation to remain in a rural area. The external issues comprised mainly of common personal issues and how they have impacted the nurses' practices.

Rural nurses are challenged with many issues related to economic status. This implies that it is highly likely that receiving sufficient income to meet the basic needs of their families should motivate rural nurses to remain in the area. The research data reveals that economic factors such as promoting equality in the salary payment inclusive of the overtime and entitlements would have the greatest impact on the nurses' motivation to remain or even go to work at rural centres. In the next few pages, these sub-themes will be explored, especially how the rural nurses' incomes affect the effectiveness of their work in relation to their allowance payments.

In addition, the social status of the participants includes such factors as culture and accommodation. A typical Papua New Guinean has two different but connected influences on their major cultural ways of being, i.e. traditional cultural beliefs (over 1,000 different) and religious beliefs (over 200) (Levy, 2005). These different socio-cultural belief systems have a considerable impact on health service delivery; this is because some contradict certain health practices, while others support such an approach. Cultural beliefs are concentrated in rural areas of PNG and are highly preserved and practiced daily. Different communities have their own beliefs and practices that are very different from each other, some of these practices have negative impacts on health care. These cultural practices are based on their ancestral beliefs and even though the current population lacks evidence to strengthen their beliefs, they are still 
conservative. Having these strong cultural ties means it is a struggle for health workers to introduce rural dwellers to health care, a lack of formal education with less western influence worsens this situation.

With these different types of beliefs, it became clearer from the data that it is a challenge for nurses to identify the patients' individual beliefs and address the health issue using an appropriate medical approach to avoid any cultural or religious encounters or disagreements. The participants also noted accommodation as another significant social factor that affected their situations at rural health centres. Accommodation is therefore examined, and more specifically punctuality and family issues, with their justifications on the needs of addressing adequate accommodation issues in rural areas.

\section{Income}

The second most highlighted sub-theme to emerge from the data is income. Overall, health professionals' incomes are lower than other public servants in PNG but within this category of professionals, the participants themselves mentioned that nurses get the lowest wages. However, the higher the hospital level, the higher their staff salary, meaning all nurses working in urban hospitals earn high salaries compared to their rural counterparts. Also, rural nurses miss out on most of the entitlements and allowances because of several reasons highlighted by participants themselves. For instance, MN1 explained that 'we often missed out from our extra allowances and the main reason is the lack of funds'. Whilst N8 pointed out that 'lack of earning our allowance is because the management system is weak in submitting our reports to PHA on time'. These are the two main causes of workers earning poor salaries and that directly demotivates health professionals' choices of rural locations. This section will discuss the rural nurses' incomes and further investigate the government and church related organisations approaches regarding nurses' salaries.

Although the nurses with similar positions right across PNG are expected to be paid on a similar rate, nurses still experience differences in their salaries. Interviews from rural nurses both in the 
government and church related hospitals revealed the nature of their salary payments and their associated difficulties. For instance, N5 (from a church related organisation) stated that: 'our salary is funded by the government, but it goes through the church organisation which is deducted to pay other church workers and we receive lower than what we are entitled too'. Hence, even when assisted by government funds, the pay of rural nurses in church run health centres remains less than the pay of nurses working in non-church run centres. This was more precisely explained by her colleague N6 who stated that 'the salaries of those nurses with similar positions as mine serving in the rural areas of the government related hospitals is triple my salary but I serve with my heart for humanity'. As her perception of the salary difference, she meant that church workers earn lower than their counterparts who are serving rurally in public health facilities which is the result of deductions not applicable to public health workers. Moreover, the salary difference is highly likely related to the provision of accommodation in church related health facilities (discussed in the next section).

Despite, earning more than church workers, public nurses still complain that their salary is too low to meet most of the basic needs such as suitable rental accommodation near health facilities. For instance, N2 stated that 'our salary is very poor'. Furthermore, after a salary comparison between rural and urban nurses' salaries was made by MN3, he explained that: 'My salary as a rural nurse is lower than the urban nurses because we missed out on most of the allowances including the overtime'. This clarifies that the urban nurses earn higher salaries because they are paid out inclusive of different allowances that the rural nurses do not receive. This implies that their rural counterparts earn less due to a lack of allowance payments. This is problematic for them, because working in a rural area brings additional demands on an already low salary, as the following participant explained:

I find it so stressful to meet the tuition fees of my children because my salary is very poor and insufficient. I often get loans and most of my salaries are deducted, the little that I earn is used for food, transport, and electricity bills. Poor salary has [a] negative influence on my job performance (N1). 
As mentioned, the salary for rural nurses is often insufficient to meet most of their basic needs. They are challenged every day to provide for their family. Yet although public nurses in rural areas receive their salary straight from the government they still expressed that what they earn is insufficient.

In addition, the participants complained of a lack of allowances and overtime payments. One of the participants stated: 'we are faced with difficulties of getting our shift loads and overtime payment on time and when it's paid we get less than what is expected' (N2). Another participant reinforced that:

Sometimes we are not paid on extra hours that we do especially on weekends and public holidays. Also, we hardly receive incentive or other rural allowances such as the hardship allowances which is compulsory payment for all rural nurses. (MN2)

On the same note, participants N5 and N6 concurred that 'we hardly receive any hardship allowances'. Thus, from at least some of the participants' viewpoints, rural nurses' compulsory allowances are less valued because less attention is given to that area. Furthermore, as another participant explained 'most nurses missed out on [a] hardship allowance and further suffer from the deduction of their leave entitlements' (N8). As a result, rural nurses are faced with financial issues because of low salaries and low remunerations. Moreover, their entitlements and allowances are deducted when paid, which worsens the nurses' financial hardship.

Salaries are generally perceived across the board as being insufficient, rural nurses expect their entitlements to be consistent to sustain themselves while serving, but many such nurses often miss out on this option. Yet, as previously indicated in this chapter, rural workers are faced with different challenges such as, working in unsafe environments, distance from main centres, lack of equipment, lack of nursing support from managers and more. Lack of all these highlighted factors are some of the important issues and why rural nurses should get remuneration to meet those costs, but currently do not. Low salaries, inconsistence allowances, and a lack of 
entitlements has a significant impact on rural nurses' job performance which directly has a negative impact on them finding suitable rental accommodation.

\section{Accommodation.}

Accommodation is very difficult in rural areas of PNG. There are very limited accommodation options in rural areas. Hence, accommodations provided is very expensive for nurses with poor salaries. Thus, most of them prefer living in their home villages. Subsequently, health professionals prefer working in urban locations where accommodation is more convenient. As in a previous example, this section will discuss the accommodation issue based on two different organisations (i.e. church and state-run health care institutions) and how it has impacted the lives of individual nurses. The discussion is based on the participants' point of view and the effect that accommodation has on workers, specifically on punctuality, attendance, and family matters.

\section{Government health facilities.}

Nurses' punctuality in their duties is very important to rural populations. The rural population live in isolated communities and reaching the nearest health facility takes hours or even days for most people. As such, patients prefer to be served as early as possible to have sufficient time to travel home. However, most health facilities often open later than the normal opening hours. For example, N2 reported that 'the foremost factor for nurses' punctuality is the accommodation. For instance, I live in my own village and travel two hours to work every day'. Parallel to that N8 agreed that 'my punctuality is very poor because I travel hours to reach the health centre', MN1 concluded that 'poor punctuality from my colleagues has demotivated me to practice here'. Clearly large travelling distances to work because of accommodation issues is a significant factor in rural nursing. Although public nurses are provided with an accommodation allowance, it is very insignificant. In part, this is because although nurses are concerned about punctuality, there is little they can do to help the situation of having adequate accommodation. 
Moreover, nurses live in different communities which are isolated from each other and the health facility, as N1 clarified: 'I provide my own accommodation which is a far distance from the health facility'. Subsequently, although nurses provide their own accommodation, those accommodations are in isolated locations and it takes hours to reach the hospital. Thus, they are often late and even absent for duties because of the poor road condition, lack of transport, and long distances to travel to work. Nevertheless, nurses are usually eager to serve and wish they were provided with more suitable accommodation so that they could be punctual to serve the local people in better ways.

It seems that most government workers would love to be provided with suitable accommodation, but it is apparent that accommodation is not part of the employment conditions of rural health professionals. As one of the participants (who is the hospital representative) explained: 'Accommodation is not a term of employment in a nursing career but we need it especially those of us serving in rural locations because most of us live and travel miles to work' (MN2). A similar statement was given by his colleague who reinforced that 'being provided with the accommodation for health professionals particularly the nurses is impossible, and most of us lives in [distant] villages' (MN1).

\section{'Church' health facilities}

However, while nurses in government related health centres struggle for accommodation, their counterparts in church organisations gave positive feedback on accommodation. One of them stated that 'church related health facilities provide accommodations while public nurses lacks this opportunity' (N5). Moreover, another participant stressed that: 'We are provided with accommodation while most nurses struggle to have such and that is one of the main motivating factors' (N6). Nurses in church related facilities agreed that it is their privilege to be provided with accommodation. Indeed, one of them stressed that:

The advantage of working with the churches is that accommodation is provided. Though [the] salary is poor, having accommodation satisfy me more. Not only were we given the accommodation but we are also provided with furniture and 
cooking appliance including some basic needs which has motivated me to continue serving in [the] church (N6).

This implies that despite the poor salary and lack of allowance, being provided with convenient accommodation satisfies nurses more than anything. The church related health facilities provide accommodation for the nurses and punctuality is therefore not an important issue for them. They feel privileged to have such opportunities despite the challenges that they are faced with in terms of income. Church workers are subsequently less attracted to government health facilities because they would not have the chance of living in a house free of rent. Government related workers provide their own accommodation compared to their church counterparts. Hence, punctuality is an issue with the public facilities more than the church facilities. Despite having low salaries, church nurses feel privileged to be in such an organisation in terms of convenient accommodation and are less attracted to government health facilities because of the accommodation. Not only does accommodation improve punctuality, it attracts and retains nurses especially those with family.

In addition, the needs of the family have a great influence on the health workers' choice of their location to practice. For many participants it appears that the family's choices and responsibilities are the core of any decision making for location of practice. Health professionals want the best for themselves, as well as, their children in terms of enhancing their education. However, this is impossible to achieve in rural locations where access to such vital services as schools and other social supports such as shops, banks, and post offices are limited. Lacking such social support has negative impacts on nurses' motivation and their job performance. For instance, N5 stated that 'I do not like working in rural areas because of my children's education'. Similarly, MN1 agreed that: 'One of the main factors that demotivates my interest in [a] rural location is my children's education'. Arguably, a lack of suitable facilities for children's education is one of the main reasons why rural areas lack nurses. The parents want the best education for their children and most Papua New Guineans believe that the best education is only provided in 
urban centres. As such, parents prefer working in urban centres so that their children can get the best education.

Parallel to that, nurses also expressed that their chances of enhancing their knowledge is very slim in rural locations. For instance, MN1 reported that I love working here and would appreciate [it] more if we are provided with more opportunities to further our studies such as having sufficient training equipment for our own staff'. On the same note, participant N9 reinforced this by stating that:

Most of my colleagues have chosen urban locations to have access to different study opportunities while I prefer rural areas to serve my community...I have worked for over ten years here, my work performance is very good but I do not get an opportunity to further my studies in order to upgrade my knowledge. I wish the management could consider such motivating factor[s] because lacking these opportunities demotivates my interest in performing daily duties.

Moreover, another CHW participant (N8) stated that 'l love attending short courses but never was I given such [an] opportunity, despite this I still serve for the love of my community'. Furthermore, N2 who is a RN reported that 'this rural hospital [is] comprised of a large population of nurses with certificate and we lack nurses with diploma and bachelor qualification in nursing'. It is clearly explained by a $\mathrm{RN}$ that most of the nurses practising in rural areas are CHWs who lack in-depth knowledge. This was acknowledged by a CHW who explained that they have the greatest desire to further their knowledge which is not often provided but they are still serving and this is because of their community.

It was stated in the beginning of this chapter that the majority of the nurses in rural areas are CHWs who are less skilled than RNs. This means that the rural health facilities are operated by nurses who critically need knowledge enhancement to provide quality health care because they practice based on observation from experienced workers. Moreover, participants noted that working in rural areas is a barrier to their children's quality education and simultaneously it 
narrows their chances of study opportunities. It is highlighted that family obligations and nurses' study opportunities have a considerable influence on health workers' motivation and choices, but it should be noted that this is nearly always in conjunction with cultural issues.

\section{Culture}

Culture in this study is closely related to individual and collective belief systems. There are two main beliefs that are highlighted by this study, which are traditional and religious. As highlighted in the introduction, PNG has a variety of cultures that are quite different from each other. In cultural practice, different beliefs are exposed which can be based on tradition or religion. As such, nurses must take extra precautions in their practice to apply a suitable approach.

\section{Religious}

An overwhelming population in PNG are Christians, within broad based Christian religion belief systems and practices such as Seventh-day Adventist, Methodists, Lutherans, Catholics, and many others (Cultural Atlas, 2018). Some of these religious beliefs are obstacles to health care, as is the case in the Catholic religion where it is against their beliefs for the women to take family planning medications or injections, based on their interpretation of the Bible, however with no restriction to any diet. In contrast to this belief, other Christians such as the SeventhDay Adventists church allow females to take family planning pills and injections while it restricts both genders from eating certain protein food such as, pork, prawns, or shrimps, including any related product based on their interpretation of the Bible.

Furthermore, the Bible that each denomination uses is slightly different from each other with different interpretations that define their beliefs. As such, there are often religious restrictions to be observed. This is because accepting or rejecting of certain medication and treatments or other medical help depends on the beliefs and practices of certain denominations. For instance, as MN2 explained: 'some mothers tell the nurses that their religion does not allow immunisation, while others belief that immunisation makes babies sick, and they reject 
immunisation for their babies'. Yet what is restricted by one religion may be accepted by others, and therefore nurses must be cautious in approaching individuals. When they do so, many find it better to try to incorporate their patient's religious beliefs (Figure 15) and practices rather than ignore them.

However, nurses who socialise with the community tend to have a broader idea of the religious beliefs and appropriate practices. Subsequently, when providing medical assistance, they incorporate the health care into their religious beliefs. For instance, for those who believe that immunisation makes their baby sick, the nurses especially those who share similar beliefs pray with them before they administer immunisation or medical assistance to patients as shown in Figure 15. N6 clarified that 'we respect their beliefs and practice in an appropriate manner'. Nurses employ different approaches to provide medical assistance and one of the possible approaches is by incorporating both practices.

Figure 15. Nurse incorporating religious beliefs into health care. 
In addition, the church governed health facilities practice according to their religious beliefs compared to public health facilities. N6 who is a church participant stated that 'we are govern[ed] by church principles'. Different church organisations do incorporate church beliefs into their practice and what is forbidden by their beliefs is not practiced within their health facilities. For instance, the Catholic health facilities do not provide family planning (apart from 'natural methods'), and contraceptive pills to mothers whereas the Seventh-day Adventist related health facilities provide these to any mother who prefers getting family planning help. Problems associated with these differences in health related practices tend to detract from the work of rural nurses who may become despondent and demotivated to continue working under such restrictions.

\section{Traditional}

Apart from the effects of different religious denominational belief systems, the rural population exhibits varieties of cultural differences alongside their religious belief systems. N6 explained that 'cultural issues is our everyday challenge, this is because some cultures accepts medical advice while other[s] prefer their own beliefs'. This means that some health practices that are forbidden by some cultures may be accepted by others, which is obviously a challenge for the rural workforce. For instance, N9 reported that 'people reject TB and HIV treatment believing that their cultural medicines will cure these diseases'. In addition, N6 mentioned that 'people blamed sorcery for illness that worsens and refuse further medical treatment and request patient to be taken home for traditional medicines' as shown in Figure 16. With such strong cultural preservation, it is very difficult to convince them to accept medical diagnosis and treatment and it is a tremendous challenge in the current situation where education in rural areas is very poor. Obviously, the rural population has strong bonds with their culture with less influence from the outside world, perhaps with the exception of Christianity (Cultural Atlas, 2018). In addition to this, they also have their religious beliefs which sometimes even contradict cultural practices while others support their beliefs. Such confusion therefore presents many problems for rural health nurses. 


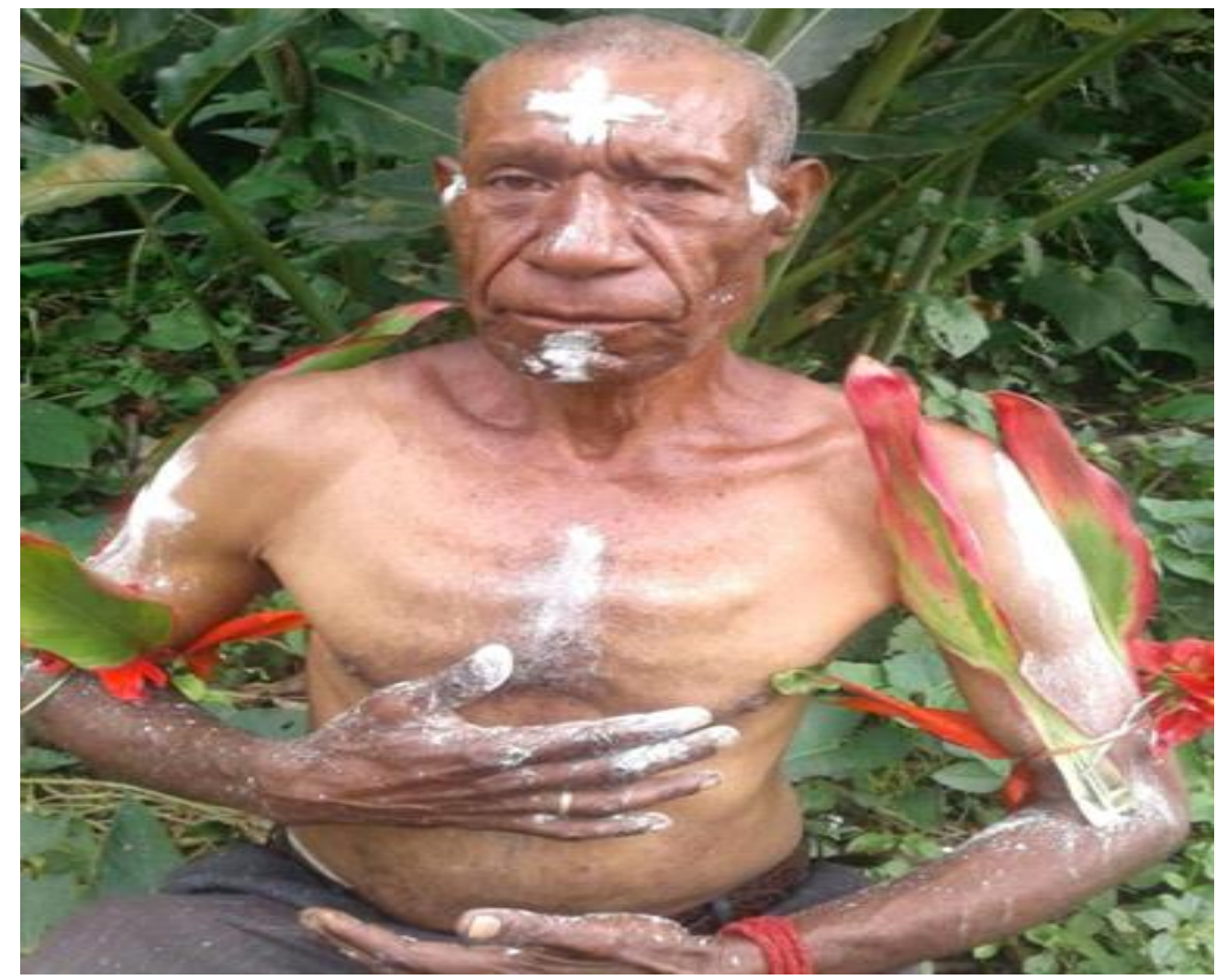

Figure 16. A man using leaves and limestone powder on his body as a traditional approach for recovery

The rural areas of PNG are more absorbed in traditional beliefs, and as a result they often overlook medical advice and diagnosis. This tends to demotivate health workers such as nurses in rural areas, because it makes their job considerably more difficult and unsafe. For instance, as participant N5 reported, 'people blame sorcerers for different illness and refuse medical treatment'. This was further elaborated by another participant who stated that ' $a$ lady was very sick and confirmed typhoid and HIV Aids positive after all the tests. However, the relatives and the patient herself blame the sorcerers and took the lady home to use traditional medication for [a] cure' (N1) as demonstrated in Figure 16. Another participant was much more explicit: Culture is very important, people still believes in their cultures...sick people are not allowed to be seen at hospitals because relatives blame witchcraft for any illnesses and believe that the traditional medicine is the only helpful approach... relatives also requests us to release admitted patients to be treated at 
home using traditional medicines, with respect to their culture we do what we can such as administration of pharmaceutical drug and provision of health education before releasing patients. Some do return when [the] health conditions worsens but most refuse because of the distance (N5).

The two participants agreed that the medical treatments are available but the patient's rights are as valued as medical help, and so although staff give their best care possible, there are still problems associated with cultural belief systems. These problems extend into areas such as delivering health education and pharmaceutical drugs, as another participant reported: 'People still reject health education because of their culture' (N6). This was supported by MN3 who agreed that 'the use of traditional medicines impedes people from seeking medical help at their earliest convenience'. Thus, as N9 concluded, 'most of the deaths in rural areas are caused by prioritising cultures over medical help'. The participants noted that cultures are highly valued and traditional medicine is often the first option people choose to treat illnesses. This practice is most often unsuccessful and, as mentioned, is one of the major causes of rural deaths. The effect of such difficulties on health workers such as rural nurses is therefore one of gradual disillusionment and uncertainty over their effectiveness in their current position.

Socioeconomic factors are focused mainly on external issues but have significant impact on the nurses' motivation to remain at or leave a rural posting. Although the salary for all nurses is equal, the organisation that they work in and the location of their practice has the greatest influence on their salary budget and accommodation. In addition, cultural and religious beliefs must be respected throughout the delivery of health care to prevent any cultural encounters, and this may often add extra strain on already overworked rural nurses.

\section{Summary}

The study explores a variety of issues as featured and highlighted in this chapter. Some of the struggles that PNG rural nurses face in delivering effective health services in rural areas are due to internal factors, and some are due to external factors. The varieties of the main issues were 
categorised into six sub-themes which are again grouped into two significant themes; safety and socioeconomic factors. The safety theme comprised of three sub-themes; basic amenities, infrastructure, and human resource. These sub-themes focused on issues related to work environment. Whilst, the socioeconomic theme comprised of three sub-themes income, culture and accommodation which are grounded on issues outside of the work environment. Because nurses from two different organisations (i.e. the government nurses and the church nurses) participated most (but not all) of their views were similar, therefore, they were generally discussed throughout the chapter. However, all the issues identified were from the rural nurses' view in general and it has great impact for recruitment and sustaining of nurses for rural health facilities throughout PNG. These issues will be revisited and addressed again in Chapter Five. 


\section{Chapter Five: Discussion}

\section{Introduction}

The findings of this study of Papua New Guinean rural nurses highlighted some of the vital factors in attracting and retaining nurses in such areas; it also revealed a significant number of non-sustaining factors that would generally have the opposite effect. In this regard, the study has revealed a significant number of motivational and demotivational factors. In general, rural locations seem less appealing to most health professionals in Papua New Guinea (PNG); however, local nurses from the surrounding villages often make sacrifices and face these challenges for the sake of their people and under the right conditions, i.e. with the right motivational factors, more nurses may take up rural nursing in PNG.

The study has shown that while there are many challenges, safety and inadequate health infrastructures in the work environment were the participants' greatest concern. Nurses often take risks by practicing within these unsafe work environments, sometimes with a lack of entirely sufficient knowledge, but their efforts are frequently overlooked by the authorities in terms of their income and entitlements. This means that practicing in the rural environment is therefore a disadvantage to most of these nurses. These different aspects, specifically the significant issues in regard to the findings of this study, are now presented and examined in this chapter with recommendations for change or improvement.

The aim of this study was to identify these sustaining factors that enable nurses to work in the rural areas of PNG. In doing so, it also revealed the motivating and demotivating factors that affected existing rural nurses to remain or consider leaving their rural practice. Nurses are obviously demotivated to practice in locations where most of their expectations are unmet and that is obviously a significant issue in rural locations. Yet despite these challenges, some nurses prefer rural locations for various reasons, many of which seemed to be associated with a willingness to forgo individual needs to assist the overall needs of the local community. 
Subsequently, while practicing, Papua New Guinean nurses in rural areas are challenged with various issues every day that may either motivate or demotivate their interest.

To strengthen the, two research articles from PNG that were related to the aim were reviewed and supplemented by other similar literature taken from worldwide sources. The nurses' motivating and demotivating factors highlighted in those studies were categorised into two main clusters of extrinsic and intrinsic factors. The extrinsic factors centred on the working environment such as career development, supervision, incentives, health infrastructures, and salary while the intrinsic motivation was based on local environment and personal factors. Local environment is inclusive of accommodation and culture while personal factors where the rural background, family, generational gap, and gender. From this literature review, it was noted that there was limited literature related to rural nurses' motivation in rural PNG, which supported the need to do the research, and the aim of the thesis.

To achieve the aim of the study, semi-structured interviews were conducted with 10 existing rural nurses. The interviews were primarily focused on sustaining factors, but quickly came to include the motivation and demotivation aspects of nurses' work in rural health practices. The data collected was translated, transcribed, and analysed using thematic analysis. A variety of issues emerged from the data that were categorised into six main sub-themes of human resources, basic amenities, hospital infrastructures, income, accommodation, and culture. These sub-themes were further categorised into two main themes of safety and socioeconomic factors.

\section{Safety}

The study highlighted that communicable diseases are common in rural areas of PNG and both the health workers and patients are exposed to several diseases that are highly contagious. This means that patients who report to the health facility may present with numerous communicable diseases that may affect the safety of both themselves, others and the nurses. As such, it is very important to promote health and hygiene in the health practices specifically 
in rural areas to prevent the spread of these diseases. However, as Wurie et al. (2016) highlighted, most of the developing countries are challenged with unreliable electricity and a lack of adequate water supply which are the foremost amenities to promote health and hygiene. PNG is no exception to this issue; indeed, in PNG this issue is extremely prevalent (Asian Development Bank, 2012). Lacking these vital amenities enables the rapid spread of cross infection within the health facility from patient to patient or nurse to patient. Therefore, it is vital for nurses to protect themselves, as well as patients, from spreading infections through simple measures such as the application of efficient hand washing and other hygiene related procedures. This is only possible if there are adequate supplies of clean water and associated resources.

Since rural areas are faced with various safety issues, water in turn plays a vital role to help reduce the risks by promoting safe practices. Sanitizing the areas of the practice reduces the build-up of microorganisms on the surface and that is accomplished only through water and other disinfectants (Provincial Infectious Diseases Advisory Committee, 2012). Water acts as the first line of defence for nurses and patients against any infections and it enables them to practice in a clean environment. However, this study noted that rural health facilities lack a consistent water supply and rely on rain water collected in a tank. Yet tank water can quickly become stagnant and accumulate dirt which is contaminated and contains varieties of microscopic bacteria. Instead of reducing the micro-organisms, it adds more to the already existing organisms. Thus, patients may present with one illness but leave with another (as was expressed in the findings in Chapter Four under health service infrastructure).

Reliable electricity plays a vital role in health care delivery during the night in rural PNG. Similar cases of patients seen during the day are seen in the facility at night, as reported by nurses, but because of the unreliable electricity supply the patients often have to wait until the assessing equipment is charged or there is enough light to do the job. This problem is a worldwide one. Adair-Rohanie et al. (2013) noted in their study of the health facilities in rural locations of SubSaharan Africa that there too, nurses are experiencing similar issues of unreliable electricity; 
thus, health care is often delivered in dark environments and the most affected are the poor vulnerable people who are patients at the health centres and aid posts. As in Sub-Saharan Africa, participants in this study mentioned that health care is delivered in dark environments because workers have no choice when a patient's life is at stake. Such an approach is similar to PNG, despite the fact that PNG is yet to fully identify the consequences of such unsatisfactory practices, it is presumed that the impact is significant.

A lack of consistent basic requirements such as an adequate water and electricity supply has been shown to be a major handicap to the delivery of safe health care around many developing nations, as both inadequate cleanliness and hygiene practices, and also an unreliable electricity supply, enables the spread of bacteria especially when practising in dark environments, as frequently mentioned by participants. These needs are vital for hygiene and prevention of disease, and so the responsible authorities must initiate possible alternatives such as providing solar lights or fuel generators and provide chemical disinfectant to treat tank water. These are relatively small and simple measures, but they would make a substantial difference to the work of rural health workers such as nurses in PNG.

\section{Health Services Infrastructure}

In addition to safety, this study reported that rural areas in PNG are in critical need of better health infrastructure. Health infrastructure includes hospital buildings and transport. Wellestablished and maintained hospital buildings would help to prevent the spread of infection and diseases because without these it can be a hazardous workplace for both the patients and workers in regard to their health and safety. Studies in other countries, developing or otherwise (Juan, Cheng, Perng, \& Castro-Lacouture, 2016) make similar claims. Since workers practising in rural areas are vulnerable to various occupational safety hazards, they need proper health infrastructure to protect them against any occupational incidents. As evidenced in the findings of this study, and although these infrastructures are vital in rural area practices, they are often overlooked and rural workers struggle to deliver health care in buildings that are badly maintained, and badly supported by sufficient local transport arrangements. 
The problems associated with poor hospital buildings a major issues highlighted by this study. Many of the hospital buildings were built in colonial days with poor refurbishment since they were first opened over 40 years ago (as reported by several participants). This problem has been recognised around the world, i.e. lack of maintaining hospital buildings is a significant hazard to rural area nurses in many remote communities such as those in Australia (McCullough, Williams, \& Lenthall, 2012). Due to lack of maintenance, it was reported that the buildings have deteriorated with broken walls, windows, roofs, and hospital furniture (like patient beds). Hence, these problems have created an unsafe practice environment for nurses and their patients alike. Protecting the nurses' health and safety by creating a safe workplace is the responsibility of the employers (National Health and Medical Research Council, 2002). This means that the issue of poor building infrastructure needs to be addressed by the Papua New Guinean health sector for the safety of its workers. In fact, this was acknowledged by the PNG health sector and a policy was developed specifically for the rural building infrastructure in the PNG community health policy post in 2013 (National Department of Health [NDoH], 2013b), but while these issues have been acknowledged by the health sector, little has been done to address the problem.

In addition, many rural health centres and hospitals lack transport infrastructure. This study highlighted that rural health facilities are isolated from the main government services and base hospitals. As such, these health facilities need reliable means of transport to meet all their health needs and to transport health workers to their places of work. However, nearly all of the participants in this study expressed their concern over the struggle that they face with the poor road infrastructure and lack of transport. They specifically noted the lack of ambulances to transfer severely ill patients from far flung villages deep in the hinterland to doctors in the provincial hospital. As a result, some patients die on their way to health centres or base hospitals. As suggested earlier, lack of access to urban health facilities for rural residents was a challenge in Australia where it has been reported that a lack of transport was one of the major barriers to adequate health service delivery (Council of Social Services of New South Wales, 
2012). If lack of transport is a challenge to the rural areas of some developed countries, then it is highly likely to be considerably worse in developing nations such as PNG. This is borne out by a similar issue with transport difficulties in Kenya which has created rural areas that are unappealing to nurses and they are often highly resistant to working in such areas (Mullei et al., 2010). These two studies from Australia and Kenya imply that besides road conditions, transport infrastructure is vital in rural areas in both developing and developed countries. Although PNG may have issues with both the road and transport infrastructure, having a reliable form of transport is vital for rural health care.

It is noted that proper health infrastructure protects the health workers' safety which is simultaneously beneficial to the patients' health care. Practising in well maintained buildings would help to protect nurses from physical injury and occupational diseases, as would having reliable modes of transport to avoid preventable deaths and get health workers to the health centres to practice in an efficient and timely manner. Subsequently, adequate hospital infrastructure plays a vital role in the health and safety of rural nurses including the rural population, and the Provincial Health Authority (PHA) must develop their own policies based on the NDoH policies targeting these vital areas. These policies must be discussed with the Local Level Government (LLG) to develop appropriate strategies to address those health infrastructure issues within different rural health facilities.

The rural health facilities in PNG are known to be isolated from the nearest base hospital with the highest rate of life-threatening diseases. As such, most referred patients do not seek further help from doctors or nurses at more advanced health care facilities due to a lack of transportation, poor road infrastructure, and financial challenges. These barriers have forced nurses to treat such critical cases in the rural health facilities to the best of their knowledge. Such situations drive nurses to perform outside their scope; although they know that the possibility of medical error is large they still take the risk when the health of the patient is at stake. This approach is inevitable unless the living standards are improved and transportation is 
provided for nurses to all rural health facilities. These challenges show that it is vital to have more skilled nurses in rural health facilities.

\section{Human Resource Investment}

The literature review emphasised that most rural nurses prefer to enhance their education but this opportunity is very slim for workers in such locations. However, nurses with less knowledge are a significant hazard to patients' health (McCullough et al., 2011). This means that study enhancement to further knowledge and skills is vital for rural nurses to provide appropriate care and the lack of this will have a negative impact on their practice. Moreover, Eslamian, Moeini, and Soleimani (2015) who conducted a focus group descriptive-exploratory research with 39 participants from Iran reported that continuing education for nurses is a vital competency in a nursing career and is also a component of professionalism in the role as nurses. Providing education to nurses not only equips them with required skills but it further develops them professionally.

Nurses require several skills through further study therefore it is essential for success. Lack of training, specifically for the rural nurses, can have a negative impact on a patient's care (Eslamian et al., 2015). In the absence of other health professionals, rural nurses perform complicated procedures to ensure their patients receive adequate care. In some instances, they lack knowledge and sometimes practice beyond their scope when a patient's life is at stake. Yet, considering the context of their practice, it is essential that rural nurses further their education. Advancing their studies would therefore broaden their knowledge and would enable nurses to attend to any case with increased confidence. This study has highlighted that further education is vital for rural nurses and while this issue was identified in two previous PNG studies (Jayasuria et al., 2012 \& Razee et al., 2012) little to nothing has been done to improve the rate of investment. However, the context of their practice reveals the importance of nurses furthering their studies. 
A policy was in place for the health workforce education but it was poorly coordinated which has resulted in staff lacking the relevant skills to match the requirements for the health service delivery in rural PNG (NDoH, 2013a). This implies that the developed policy lacks implementation. However, the health sector has planned to train and recruit sufficient skilled and qualified nurses to rural locations, but this is yet to be fully accomplished at the current stage. Here the PNG health department suggested to increase the rural nursing staff numbers by recruiting externally from urban locations. Yet while this appears to be one solution to the inadequate nursing stock, trained nurses are still not attracted to rural areas, most likely for the reasons that are highlighted in this study.

To address the issues of having sufficient numbers of qualified nurses in rural locations, other countries use different approaches to provide nurses with adequate further study opportunities. For instance, Canada provides incentives in the form of tuition fees for rural nurses' education through online studies (MacLeod et al., 2017). Also, rural hospitals in Australia create partnership programs where each nurse shares knowledge and skills in their area of interest (Shoo, Lawn, \& Carson, 2016). These two studies in developed countries revealed that education enhancement is provided specifically to existing rural nurses to develop their skills, and that this approach has been reasonably successful. What PNG health can learn from these examples is to recruit existing PNG rural nurses whilst at the same time providing suitable study enhancements. Indeed, the Canadian and Australian examples showed that this investment in the current stock will have positive impacts on the rural majority over time.

The lack of qualified health professionals in rural areas can only be addressed through providing better educational opportunities to existing rural nurses. Most of the factors outlined by the study for rural nurses' motivation remains unmet meaning the situation is uninviting for urban nurses. Subsequently, to recruit and train external nurses to work in rural areas is an unpredictable aim, and it remains unlikely that they will find these areas appealing to work in after their training. Therefore, to be sure of having qualified nurses in rural locations, a greater 
emphasis on health workers training needs must be provided for existing rural nurses, as has been successfully done in other countries.

\section{Rural Nurses Income}

Research by Henderson and Tulloch (2008) revealed that the salaries of health professionals in developing countries are not of an expected standard, often being much lower than those in developed countries and also much less than the pay of urban nurses in their own country. It has been confirmed in Chapter Two and in the findings that the health professionals in PNG are offered poor salaries. In this category of health professionals, nurses reported that their salary is lower than any other health professionals and more importantly lower than their expenses. They mentioned that most of their daily economical and actual needs are unmet, and they struggle daily to take on financial commitments for their family. Moreover, they lack most of the rural entitlements that they should receive, as they are faced with difficulties when receiving their overtime payment on set dates; in fact, sometimes they are unpaid.

The salary for the nurses in PNG is based on the position occupied including their experience as per General Order \#3, Salaries and Allowance 13.14 (Department of Personal Management, 2012). As such despite their location of practice, nurses with a similar position are expected to earn similar salaries, as documented in General Orders. However, this policy lacks effective implementation in rural locations for reasons that remain obscure; nevertheless, rural nurses mention that they earn lower than their urban counterparts regardless of their service. This means that rural nurses' experiences and positions are not valued because their allowances and benefits are inconsistent across the health sector. This undervaluing of their skills and importance in rural health is therefore a significant demotivating factor to seeking or maintaining employment in rural areas.

Moreover, workers mentioned that they often missed out on receiving their allowance for working on public holidays and weekends. General Order \#3, 13.55(d) and (e) clarified that shift workers working on Sundays and public holidays are entitled to receive double time with a 
minimum of half a day's pay. This Act was amended in 1995 and revised in 2012 meaning the funds are provided to implement this Act. However, this Act was poorly coordinated and implemented in rural locations, as noted by the participants, and such payments are often simply not made, and frequently no reasons for these omissions are offered.

While less is done in PNG to sustain its rural workers through adequate remuneration, other countries have developed different approaches to retain their nurses through various incentives. For instance, Australia and some developing countries provide higher salaries for rural nurses compared to their urban counterparts. Both MacLeod et al. (2017) and WillisShattuck et al. (2008) note this feature as a part of the motivating factors to attract and retain nurses to areas where the need for health professionals is high. This is supported by the research of Humphreys et al. (2013), who conducted their study in the rural and remote health facilities in Australia with the main focus on rural health workers' retention, that concluded that the practice of regular and weighted salary increments for rural nurses creates an appealing environment for these nurses practicing in rural locations of the country. It also enables the recruitment process to be successful in employing more staff. Hence, the importance of appropriate salary increments for rural workers has been acknowledged by developed and developing countries, and they have invested more into this area to motivate and sustain their nurses.

As in these other countries, the Department of Personal Management (DPM) has also acknowledged the importance of rural nurses' incomes and has created policy but it lacks effective implementation. This is a well-recognised and prevalent issue in PNG, as Asante and Hall (2011) discovered. They emphasised that most of the policies and plans by NDoH in PNG are not implemented by the PHA and DHA. The lack of policy implementation as stated above is the result of various issues. One of the main reasons for this will be the cultural practice of broadening the gap for corruption (Wickberg, 2013; Wilson, 2015). This means that the wantok system issues where people whom they know are given higher priority for higher responsibilities without considering their required merit status. As a result, most of the priority 
areas are overlooked because some of the people occupying such positions lack the leadership skills to identify and develop suitable strategies to address existing issues (Kolehmainen-Aitken, 1992). Hence, the policies and plans by NDoH are weakly coordinated. Apart from poor leadership skills, the common and known issues are the lack of funds and mismanagement of those funds. The limited funds if substantially focused on low priority areas, inclusive of the administration, will have a tangible effect (Nem, 2015). This literally means that most of the allocated funds are spent on areas that are less critical thus, crucial areas in health become less prioritised and rural health facilities are mostly affected which increases workers' demotivation. Therefore, to meet the needs of rural nurses in PNG, the PHA (who are in charge of staff issues within the provinces) must focus on priority areas for motivation and one of these is being effective in implementing salary and allowances policy which has already been amended and enacted.

\section{In Summary}

Four significant issues on the retention of rural nurses and the factors that sustain, motivate or demotivate nurses to remain or leave have been discussed so far in this chapter. It has been argued that safety factors are strongly related to the work environment and this remains the foremost concern for all health workers in PNG therefore needs immediate attention. Moreover, rural nurses who serve the local population need further education to enhance their knowledge and maintain their efficiency. Since their practice is on a par with or more complex than their counterparts in urban locations their efforts must be valued in terms of consistency including proper wages and the payment of compulsory allowances. This calls for the provincial authorities to identify their roles and implement created policies. It also suggests some necessary changes to some policies that currently demotivate nurses from working in rural areas; this includes accommodation which if made satisfactory, would have the greatest impact on recruiting nurses to work in rural health. 


\section{Implications}

According to discussions with rural nurse participants in PNG and the subsequent data analysis and thematic presentation of findings, four main sets of implications and their associated recommendations are now suggested, namely those relating to safety, education, income, and policy. If implemented effectively, these recommendations should help to recruit and sustain more nurses to rural locations across PNG.

\section{Implication for nurses' safety.}

The issues of poor safety in the work environments in rural areas of PNG is an ongoing issue which needs considerably more investment. More specifically, because the different aspects of safety in the working environment was a major concern for all participants, it follows that immediate steps should be taken to address this issue. The findings reported that workers were determined to provide a health service to the people, however the obstacles were considerable. They were chiefly based on the unsafe practice environments, with the main theme of safety needs. This mainly relates to the health and safety hazards created by the health facilities themselves, including the lack of proper equipment and amenities to improve practice within a safe environment.

Unsafe working environments such as, broken beds for patients, lack of delivery trays, rusted roofs, and many more issues in rural PNG implies that urgent investment in these areas is needed. This was confirmed by all the nurses who expressed several concerns about the unsafe working environments. Reporting the same complaints over long periods of time implies that this has a negative impact on rural nurses who are the frontline health care providers because it directly exposes them to various dangers. Subsequently, providing them with the required medical equipment, adequate and safe water supplies, reliable electricity supplies, and proper hospital infrastructure will help protect them (and their patients) against any occupational injury or diseases. To create a safe working environment there needs to be a close working relationship with the different authorities and levels including the health facilities to identify the areas that are unsafe for the nurses. 
Therefore, the recommendation is for authorities and organisations to develop objectives and plans and work towards achieving the health and safety goals more effectively. This means that the plans and policies developed must be specific to the rural context with specific nursing protocols. Also, the authorities in charge, which is the LLG, must conduct critical investigations by observing the health facility, as well as, interviewing nurses to get their views and recommendations so that suitable approaches can be employed to address the issues effectively. This can then be discussed with the PHA for extra funding to implement the suggested plans. Furthermore, the hospital management must be active in their functions, they must assess the facilities constantly to identify the emerging issues and address those problems promptly with the consideration to the nurses' safety.

\section{Implication for nursing education.}

Nurses' education plays a vital role not only in healthcare staff motivation but also for patients' health. In particular, the majority of nurses in this study indicated that they prefer advancing in their studies in specialty areas such as midwifery, Paediatric nursing, and so on. However, opportunities are limited in rural locations and most nurses do not get an opportunity to do so. Thus, a majority of rural nurses remain as general practitioners with a lack of specialist skills; nevertheless, this in turn means that they can offer comprehensive general services to local communities, albeit ones where advanced skills are limited.

To achieve this aim, providing nurses with study opportunities will have a significant impact on the health of the rural population. Because rural nurses in PNG perform multiple tasks and have acquired different skills within and outside their scope, this means that they are capable of furthering their education and have a clear need to do so. Enhancing their education will elaborate their understanding of some of the obstacles that they have faced within their practices, this will enable them to practice even better to save the majority who cannot make it to a provincial base. Study enhancement for nurses enables knowledge and skill development which improves their nursing function, thus, improves health care in their respective practice 
environment. This study, in regard to nurses continuing their education, reinforces the notion that rural nurses' education is vital for their work with a rural population that is out of reach of other qualified health professionals.

The recommendation therefore is that the PHA must be more engaged in the nurses' training. This means that they should have effective communication with the LLG and the management of different rural health facilities to identify areas that needs skilled nurses and together they can set the criteria and select those nurses that meet the requirements to further their training. Therein, more investment for human resource development in health is needed more specifically for rural nurses.

\section{Implication for effective implementation of nurses' incomes.}

The issues of poor salaries for the nurses, specifically in rural areas, is an ongoing challenge. It was revealed by nurses in this study that rural nurses earn less than their urban counterparts. Yet despite the salary differences, the nurses expand and extend in their practices to deliver health care to the rural majority.

The roles are divided among different levels with specific responsibilities. Kolehmainnen-Aitken (1992) clarified that the funds are diverted to PHA to oversee the provincial staff issues by implementing the amended Act. It means that implementation of the salary and allowance Act is the responsibility of PHA. However, a well-articulated policy and plan lacks implementation at the provincial level (Asante \& Hall, 2012). This was also confirmed by this study with the inconsistent allowances for rural nurses meaning that PHA must be aware of the consequences and the impact that it will have on the workforce if the policies are not implemented. Such inactivity has affected the rural nurses financially. However, this does not prevent them from working on public holidays and weekends whenever they are rostered. This shows that rural nurses value their careers and their willingness to serve the people. 
To value such effort and obvious dedication to the health of the populace, the recommendation is that the Act must be effectively implemented with the focus on rural nurses, and this calls for the authority in charge to be active in this role. The Act is amended but lacks implementation which means that there is an obstacle somewhere. As such, the NDoH must set the health audit team to monitor how the funds are spent and why the rural nurses are missing out on some of their allowances. This will enable then to identify barrier and based on these, the specific policies should be created to suite rural nurses' income.

\section{Implications for rural nursing policy in Papua New Guinea.}

There are different policies in place for the rural areas which are more specifically targeted at the improvement of the health status of the populace. The NDoH has developed wellarticulated policies to meet the needs of rural health, such as salary and allowances. However, these policies need a sufficiently incentivised and well-motivated workforce to implement the plans. Since nurses are the main health care provider in rural areas, a real push by the entire health sector could build capacity to force policy compliance and improve health statistics in PNG. This is because the statistics that relate to nurses (as mentioned in the first chapter) reveal that there are less nurses in rural locations than urban even though rural nurses cater for over $85 \%$ of the population. Due to this unbalance, the policies and plans developed by NDoH are less likely to be implemented as urban nurses are benefiting from the distribution of resources.

As previously noted, the policies created by the PNG health authorities are generalised in their meaning and cover all nurses in general. Yet, comparing health professionals, nurses in the rural areas expand much more in their clinical practice than their counterparts in urban locations. The challenges that rural nurses face are more critical due to the location and resources and they work against those challenges to ensure the health service is delivered. Hence, although nurses throughout PNG have similar job functions, the functions of the rural nurses are significantly different than their urban counterparts due to the population distribution and 
demand. As such, rural nurses need specific policies to meet their specific needs, and a far greater degree of support at all levels.

To conclude, one of the main issues that is revealed by the study is the lack of specific policies for rural nurses. Developing specific policies for the rural nurses is therefore a vital need and must be targeted to specific (i.e. rural) locations. Therefore, it is recommended that before creating any further policy regarding rural health services, a critical investigation must be conducted in different work environments to identify and target the needs according to the population in each level. Such an investment would enable the policy makers to develop policy that will satisfy the nurses in different locations. Also, the government must fund more research centred on rural health. In doing this, it will identify various underlying issues that have demotivated nurses to practice in those locations. This will enable the creation of new policies and also stimulate some of the inactive policies to become an Act and be amended so that they can be funded for effective implementation.

\section{Future Directions for Research}

Health related research in PNG, and especially research involving the work of nurses in that country is a very rare phenomenon. It is little wonder therefore that the PNG Health Authorities and Provincial Governments have had little material to go on when considering improvements to rural health services. This research has been an attempt to redress this acute problem in at least a small way, and to identify (as in the last few pages) areas on how the vital rural health services in PNG may be enhanced, especially using the dedicated skills of the vast majority of health workers in those areas, i.e. nurses.

Yet, this small project should be regarded as only perhaps a beginning attempt at providing research that could guide health initiatives in PNG's rural areas. Clearly a great deal more research is necessary, and in relation to the role of nurses as the greatest number of health care providers in the rural areas, a great deal more research is clearly needed that involves PNG's nurses in being the main topic for study, the participants and the researchers. This will require greater support for those Papuan nurses who wish to pursue higher studies and subsequent research projects either within PNG or overseas. 
Subsequently, it is recommended that future research in rural health issues in PNG should include studies that concentrate on the vital role of nurses in PNG's vast rural areas. For example, comparisons of the operating and delivery systems of the two main supporting organisations, i.e. state and church could be made, and the roles that nurses play in these systems; the effects of well supported health care services versus poorly supported ones; and most certainly, further research, possibly a national survey, or further qualitative research, into the ways in which nurses might be encouraged to work and remain working in the rural areas of PNG.

\section{Limitations of the Study}

Well recognised credibility and reliability requirements were applied to this research, in other words the collected data was of sufficient depth and relevance to the topic, clear decision trails are apparent in the interpretation of data, and rich and thick verbatim descriptions of participants' accounts were used to support the findings. However, there are several limitations to this study that affect the degree of generalisation or widespread meaning that may be possible, although not necessarily the reliability and the credibility of the study. As a qualitative Masters project, this is a well-recognised issue in this study.

These limitations are as follows. Firstly, this study was carried out in different districts of the same Highlands province. As previously stated, PNG has two main regions; Coastal and Highlands. Although the lifestyle is different, the challenges that nurses faced in these two regions are likely to be similar. As such, the study was focused on only one region because it represented the average PNG rural setting very well. However, further study should include both regions for more specific comparison and more specific development of ideas.

Secondly, two different organisations, i.e. government and church, were included in the study. Although NDoH health policies apply to both organisations, church organisations each have specific policies grounded on religious principles rather than just on government directives. These church policies tend to be more accommodating of the needs of rural nurses especially when relating to housing needs. Due to different rules in management, future study might be 
needed when conducting an in-depth investigation to fully explore the approach each organisation's methods for sustaining rural nurses.

Thirdly, the genders of the participants were unequal which reflects the nurses. Subsequently, the study was female dominated and the viewpoints of the male nurses were limited. Therefore, future study might want to restore gender balance for in-depth knowledge on different opinions from a male and female perspective.

Finally, this study was conducted using a descriptive-exploratory method. The study explored and described the sustaining factors (and often the non-sustaining factors) for rural nurses without further specific or more concentrated information on different aspects including social and economic and other possible factors. However, this exploratory study has created a foundation for further study, and hopefully remains a useful contribution to further debates and developments of rural healthcare in PNG.

\section{Conclusion}

Shortages of nurses is a major problem in the PNG health sector and rural locations are the worst affected areas. The decline of nurses in rural locations became apparent postindependence since the introduction of the decentralised health system and it is currently a major problem faced by the country. As such, this study reviewed literature from PNG and other countries and the causes identified were found to be chiefly associated with issues regarding career development, income, infrastructure, supervision, accommodation, culture, rural background, family, the generational gap, and gender. Although these studies were relevant to rural health professionals in PNG, there remained until now a greater need for a specific study on rural nurses in that nation.

Subsequently, this study was conducted using participants drawn from the rural nurses working in often remote or semi-remote rural areas of PNG to investigate and identify the motivating and demotivating factors that affected nurses' decisions to remain or leave rural practices. For 
this study to be effective, and because it was the first of any such study in PNG, a descriptiveexploratory approach was employed on rural nurses from the church and the government health facilities. The findings of the study revealed several contributing factors to nurse shortages namely human resources, basic amenities, health services infrastructures, income, culture, and accommodation.

Based on the discussion of these contributing factors it is recommended that the health sector prioritise the safety of the working environment in all aspects, they must ensure that their staff are well protected and safety protocols for all nurses are effective. Furthermore, NDoH must investigate socioeconomic factors such as education and income and identify the barriers to why these existing policies lack implementation. In doing so, it will enable three levels, NDoH, PHA, and LLG, of the health sector to create policies that suit the rural locations which will perhaps have a positive impact on the nurses' motivation to rural health facilities in PNG. 


\section{Appendices}

\section{Appendix 1: Information Sheet}

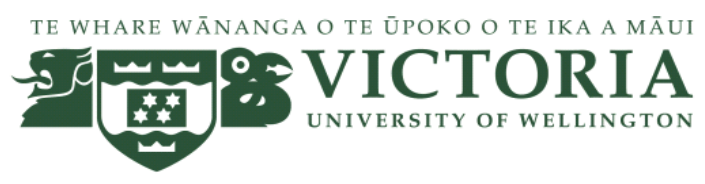

\section{Project Title: What are the sustaining factors for nurses in rural areas of}

\section{Papua New Guinea?}

Thank you for your interest in this project. Please read this information before deciding whether or not to take part. If you decide to participate, thank you. If you decide not to take part, thank you for considering my request.

\section{Who am I?}

My name is Priscilla Poga and I am a student at Victoria University of Wellington in New Zealand. I did my Bachelor of nursing at the Pacific Adventists University (PNG) and worked for two years at the country's national hospital. In 2015 I got an offer from Victoria University and was enrolled for the Masters of Health Care in 2016. I am now in the final year of my study with the requirement to complete this research project for my thesis.

\section{What is the aim of the project?}

This is my final project and I am required to do a research based on a particular health issue in my country. Therefore, in this project I have decided to conduct a research on nurses sustaining factors in rural areas of Papua New Guinea (PNG). Papua New Guinea have a vast majority of the population residing in rural areas. However, it seems that over $75 \%$ of the health professionals prefer working in urban settings. The government is currently faced with a challenge of retaining and recruiting health workers to rural locations. Therefore, this area was chosen with the aim of identifying some of the factors that might contribute to the rural nurses' job satisfaction, including some of the reasons why most nurses are reluctant to respond when posted to work in rural areas. 
This research has been approved by the Victoria University of Wellington Human Ethics Committee in June.

\section{How can you help?}

If you agree to take part I will interview you on one to one basis in a convenient environment within the clinic. The interview can either be in English or Tok Pisin based on whichever that suits you. Also, your profession will be mentioned instead of your name. The questions will be based on your job satisfaction in where you are practicing. The interview will take 25 minutes to 45 minutes or more. I will record the interview and a write up will be done later. You can stop the interview at any time, without giving a reason. You can withdraw from the study by contacting me at any point before 25th July 2017. If you withdraw, the information you provided will be destroyed or returned to you.

\section{What will happen to the information you give?}

Access to read the notes or transcript of the interview will be kept confidential between me and my supervisor. The information will be accessible through a secured password between both of us. This information will not be used for other purposes without seeking your consent. The interview transcripts, summaries and any recordings will be kept securely and destroyed five (5) years after the research ends.

\section{What will the project produce?}

The information from my research will be used for my Master's thesis and also will try publishing.

\section{If you accept this invitation, what are your rights as a research participant?}

You do not have to accept this invitation if you don't want to. If you do decide to participate, you have the right to:

- $\quad$ choose not to answer any question;

- $\quad$ ask for the recorder to be turned off at any time during the interview;

- $\quad$ withdraw from the study before 25th July 2017;

- $\quad$ ask any questions about the study at any time;

- $\quad$ receive a copy of your interview recording;

- $\quad$ read over and comment on a written summary of your interview; 
- be able to read any reports of this research by emailing the researcher to request a copy.

If you have any questions or problems, who can you contact?

If you have any questions, either now or in the future, please feel free to contact either:

Student:

Name: Priscilla Poga

University email address:

pogapris@myvuw.ac.nz

\section{Supervisor:}

Name: Dr Martin Woods

Role: Senior Lecturer

School: Nursing, Midwifery \& Health

Phone: 00644635034

martin.woods@vuw.ac.nz

\section{Human Ethics Committee information}

If you have any concerns about the ethical conduct of the research you may contact the Victoria University HEC Convener: Associate Professor Susan Corbett. Email susan.corbett@vuw.ac.nz or telephone +64-4-463 5480 . 


\section{Appendix 2: Consent Form}

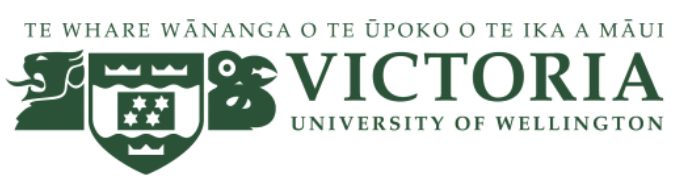

\section{Project Title: What are the sustaining factors for nurses in rural areas of Papua New Guinea?}

This consent form will be held for five (5) years.

Researcher: Priscilla Poga, School of Nursing, Midwifery and Health, Victoria University of Wellington.

- I have read the Information Sheet and the project has been explained to me. My questions have been answered to my satisfaction. I understand that I can ask further questions at any time.

- $\quad$ I agree to take part in an audio recorded interview.

I understand that:

- I may withdraw from this study at any point before 25th July 2017 (might change), without giving any reason, and any information that I have provided will be returned to me or destroyed.

- $\quad$ The information I have provided will be destroyed five (5) years after the research is finished.

- $\quad$ Any information I provide will be kept confidential to the researcher and the supervisor. I understand that the results will be used for a Masters report and a summary of the results may be used in academic reports and/or presented at conferences.

- $\quad$ My name will not be used in reports, nor will any information that would identify me.

- I would like a copy of the transcript of my interview: Yes No

- I would like a summary of my interview: Yes No

- I would like to receive a copy of the final report and have added Yes No my email address below. 
Signature of participant:

Name of participant:

Date:

Contact details: 


\section{Appendix 3: Interview Guide}

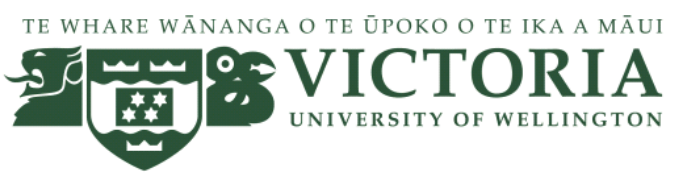

\section{Interview Questions:}

- Tell me about yourself?

- Why you chose to work in rural areas?

- What are the challenges in the work environment and how it has influenced your work and how were you able to handle these challenges?

- What are some of the personal challenges in working in this health facility and how do you cope with that?

- How does it impact your life?

- What would you suggest the health sector should do to attract more nurses to rural health facilities?

These are all the questions we have for you. Before we leave, do you have other responses or comments about the information discussed today?

I will analyse the information and I'll provide the clinic with the copy of results in approximately six months.

Thank you for participating, the information that you have provided is very important and is very helpful to me.

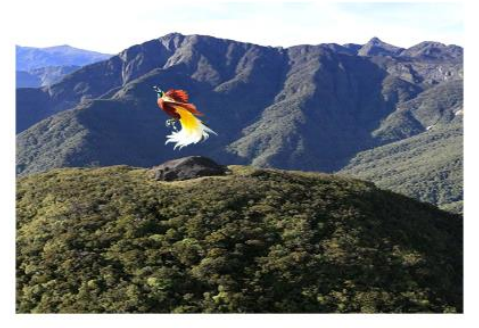




\section{References}

Adair-Rohani, H., Zukor, K., Bonjour, S., Wilburn, S., Kuesel, C. A., Hebert, R., \& Fletcher, R. E. (2013). Limited electricity access in health facilities of sub-Saharan Africa: A systematic review of data on electricity access, sources, and reliability. Global Health: Science and Practice, 1(2), 249-261. http://doi.org/10.9745/GHSP-D-13-00037

Ajani, K., \& Moez, S. (2011). Gap between knowledge and practice in nursing. Procedia-Social and Behavioural Sciences, 15, 3927-3931. https://doi.org/10.1016/j.sbspro.2011.04.396

Alhassan, K. R., \& Nketiah-Amponsah, E. (2016). Frontline staff motivation levels and health care quality in rural and urban primary health facilities: a baseline study in the Greater Accra and Western regions of Ghana. Health Economics Review, 6, 39. "https://doi.org/\%20HYPERLINK\%20\%22https://dx.doi.org/10.1186\%2Fs13561-0160112-8\%2210.1186/s13561-016-0112-8"10.1186/s13561-016-0112-8

Andrew, D. (2013, January 11). Improve package of doctors, nurses. The National. Retrieved from http://www.thenational.com.pg/improve-package-of-doctors-nurses/

Asante, A., \& Hall, J. (2011) A Review of Health Leadership and Management Capacity in Papua New Guinea. Retrieved from https://sphcm.med.unsw.edu.au/sites/default/files/sphcm/Centres_and_Units/LM_PN G_Report.pdf

Asian Development Bank (2012). Papua New Guinea critical development constraints. Retrieved from https://www.adb.org/sites/default/files/publication/29776/png-criticaldevelopment-constraints.pdf

Asian Development Bank (2014). Human health resources subsector analysis (Summary). Retrieved from https://www.adb.org/sites/default/files/linked-documents/41509-013png-oth-01.pdf

Asian Development Bank (2015). Health sector assessment. Retrieved from https://www.adb.org/sites/default/files/linked-documents/CAPE-PNG-7-Health-SectorAssessment.pdf 
Baernholdt, M., \& Mark, B. A. (2009). The nurse work environment, job satisfaction and turnover rates in rural and urban nursing units. Journal of Nursing Management, 17(8), 994-1001. http://doi.org/10.1111/j.1365-2834.2009.01027.x

Baernholdt, M., Yan, G., Hinton, I., Rose, K., \& Mattos, M. (2012). Quality of Life in Rural and Urban Adults 65 Years and Older: Findings from the National Health and Nutrition Examination Survey. Journal of Rural Health, 28(4), 339-347. http://doi.org/10.1111/j.1748-0361.2011.00403.x

Barcson, S. B. (2015). Challenges to implementing of development plans at local-level government in Papua New Guinea. Commonwealth Journal of Local Governance, (16). http://dx.doi.org/10.5130/cjlg.v0i0.4492

Blaauw, D., Erasmus, E., Pagaiya, N., Tangcharoensathein, V., Mullei, K., Mudhune, S., Lagarde, M. (2010). Policy interventions that attract nurses to rural areas: A discrete choice experiment. Bulletin of the World Health Organisation, 88(5). "http://doi.org/10.2471/BLT.09.072918"

Blades, P. (2017, March 17). HIV remains critical threat in Papua New Guinea. Radio New Zealand. Retrieved from https://www.radionz.co.nz/international/pacificnews/326821/hiv-remains-critical-threat-in-papua-new-guinea

Bolger, J., Mandie-Filer, A., \& Hauck, L. (2005). A review of capacity, change and performance issues. Papua New Guinea's health sector. Retrieved from http://ecdpm.org/wpcontent/uploads/2013/11/DP-57F-Papua-New-Guinea-Health-Sector-Capacity-ChangePerformance.pdf

Bourke, L., \& Sheridan, L. (2010). Understanding rural health - Key concepts. Retrieved from http://arhen.org.au/images/publications/chap2.pdf

Braun, V., \& Clarke, V. (2006). Using thematic analysis in psychology. Qualitative Research in Psychology, 3, 77-101 https://www.tandfonline.com/doi/pdf/10.1191/1478088706qp063oa?needAccess=true Braun, V. \& Clarke, V. (2012). Thematic analysis. In H. Cooper, P. M. Camic, D. L. Long, A. T. Panter, D. Rindskopf, \& K. J. Sher (Eds.), APA handbook of research methods in 
psychology, Vol. 2: Research designs: Quantitative, qualitative, neuropsychological, and biological (pp. 57-71). Washington, DC: American Psychological Association.

Braun, V., \& Clarke, V. (2014). What can "thematic analysis" offer health and wellbeing researchers? International Journal of Qualitative Studies on Health and Well-Being, 9. https://doi.org/10.3402/qhw.v9.26152

Buchan, J., Connell, J., \& Rumsey, B. (2011). Recruiting and retaining health workers in remote areas: Pacific island case-studies. Retrieved from http://digicollection.org/hss/documents/s19240en/s19240en.pdf

Burke, E. (2015). Being a nurse in North is challenging but 'there are salary perks'- big ones. Retrieved from http://www.macleans.ca/education/college/being-a-nurse-in-the-northis-challenging-but-there-are-salary-perks-big-ones/

Campbell, E. N. (2015). Investigating the motivation and personal traits of allied health professionals working in remote and rural Australia: The remote and rural allied health motivation and personal (RRAHMP) study. (PhD Thesis).

"https://doi.org/\%2OHYPERLINK\%20\%22http://dx.doi.org/10.14264/uql.2015.1058\%22 10.14264/uql.2015.1058"

Catholic Agency for Overseas Development. (2017). Power to be: How lack of electricity affects everything - and what you can do about it. Retrieved from https://blog.cafod.org.uk/2017/06/16/power-to-be-how-lack-of-electricity-affectseverything-and-what-you-can-do-about-it/

Chimwaza, W., Chipeta, E., Ngwira, A., Kamwendo, F., Taulo, F., Bradley, S., \& McAuliffe, E. (2014). What makes staff consider leaving the health service in Malawi? Human Resources for Health, 12, 17. https://doi.org/10.1186/1478-4491-12-17

Connell, J. (1997). Health in Papua New Guinea: A decline in development. https://doi.org/10.1111/1467-8470.00027

Council of Social Service of New South Wales. (2012). Provided there's transport: Transport as a barrier to accessing health care in NSW. Retrieved from https://www.ncoss.org.au/sites/default/files/121206health-transport-report.pdf 
Cultural Atlas. (2018). Papua New Guinean Culture. Retrieved from

https://culturalatlas.sbs.com.au/papua-new-guinean-culture/religion-7fea8435-f2e4494e-bc92-402318335682

Darkwa, E. K., Newman, M. S., Kawkab, M., \& Chowdhury, M. E. (2015). A qualitative study of factors influencing retention of doctors and nurses at rural healthcare facilities in Bangladesh. BMC Health Services Research, 15, 344. http://doi.org/10.1186/s12913015-1012-z

Department of Health in Australia. (2013). Rural recruitment and retention strategies. Retrieved from http://www.health.gov.au/internet/publications/publishing.nsf/Content/work-

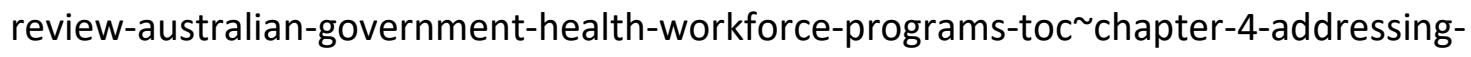
health-workforce-shortages-regional-rural-remote-australia chapter-4-ruralrecruitment-retention-strategies

Department of National Planning and Monitoring. (2010a). Papua New Guinea development strategic plan 2010-2030. Retrieved from http://www.asialeds.org/sites/default/files/resource/file/Publications_DSP20102030.pdf

Department of National Planning and Monitoring. (2010b). Papua New Guinea medium term development plan 2011-2015. Retrieved from http://www.treasury.gov.pg/html/publications/files/pub_files/2011/20112015.png.mtdp.pdf

Department of Personal Management (2012). General order \# 13: salaries and allowances. Retrieved from https://pngdpmweb.s3.amazonaws.com/documents/doc_GeneralOrder13SALARIESANDALLOWANCES4t hEdition2012.pdf

Dieleman, M., Cuong, V. P., Anh, V. L., \& Martineau, T. (2003). Identifying factors for job motivation of rural health workers in North Viet Nam. Human Resources for Health, 1, 10. https://doi.org/10.1186/1478-4491-1-10

Duke, T. (1999). Decline in child health in rural Papua New Guinea. The Lancet, 354(9186), 1291-1294. https://doi.org/10.1016/S0140-6736(99)00335-9 
Dussault, G., \& Franceschini, M. C. (2006). Not enough there, too many here: understanding geographical imbalances in the distribution of the health workforce. Human Resources for Health, 4, 12. http://doi.org/10.1186/1478-4491-4-12

Ebuehi, O. M., \& Campbell, P. C. (2011). Attraction and retention of qualified health workers to rural areas in Nigeria: A case study of four LGAs in Ogun State, Nigeria. Rural and Remote Health, 11, 1515. Retrieved from http://www.rrh.org.au/publishedarticles/article_print_1515.pdf

Eslamian, J., Moeini, M., \& Soleimani, M. (2015). Challenges in nursing continuing education: A qualitative study. Iranian Journal of Nursing and Midwifery Research, 20(3). 378-386. Retrieved from https://www.ncbi.nlm.nih.gov/pmc/articles/PMC4462065/

Essendi, H., Johnson, F. A., Madise, N., Matthews, Z., Falkingham, J., Bahaj, A. S., \& Blunden, L. (2015). Infrastructural challenges to better health in maternity facilities in rural Kenya: Community and healthworker perceptions. BMC Reproductive Health, 12, 103. http://doi.org/10.1186/s12978-015-0078-8

Expanded accommodation for medical workers (2017, February 27). Loop PNG. Retrieved from http://www.looppng.com/community/expanded-accommodation-medical-workers53301

Fowler, D, J. (2009). Cultural and Structural Barriers that Affect the Doctor-Patient Relationship: A Bolivian perspective (Honors Thesis). Retrieved from https://ir.library.oregonstate.edu/xmlui/bitstream/handle/1957/9896/Fowler.pdf

Franco, M. L., Bennett, S., \& Kanfer, R. (2002). Health sector reform and public sector health worker motivation: A conceptual framework. Social Science \& Medicine, 54(8), $1255-$ 1266. https://doi.org/10.1016/S0277-9536(01)00094-6

Gibson, J., \& Rozelle, S. (2003). Poverty and access to roads in Papua New Guinea. Economic Development and Cultural Change, 52(1), 159-185. Retrieved from https://www.journals.uchicago.edu/doi/10.1086/380424

Gill, P., Stewart, K., Treasure, E., \& Chadwick, B. (2008). Methods of data collection in qualitative research: Interviews and focus groups. British Dental Journal, 204(6), 291295. https://doi.org/10.1038/bdj.2008.192 
Global Health Workforce Alliance. (2018). Papua New Guinea. Retrieved from http://www.who.int/workforcealliance/countries/png/en/

Grujicic, M., Jovicic-Bata, J. J., Raden, S., Navokavic, B., \& Sipetic-Grujicic, M. (2016). Work motivation and job satisfaction of health workers in urban and rural areas. Vojnosanit Pregl, 73(8), 735-743. https://doi.org/10.2298/VSP140715062G

Henderson, L. N., \& Tulloch, J. (2008). Incentives for retaining and motivating health workers in Pacific and Asian countries. Human Resources for Health, 6, 18. https://doi.org/10.1186/1478-4491-6-18

Hewison, A., \& Wildman, S. (1996). The theory-practice gap in nursing: A new dimension. Journal of Advanced Nursing, 24, 754-761. Retrieved from https://onlinelibrary.wiley.com/doi/epdf/10.1046/j.1365-2648.1996.25214.x

Hongoro, C., \& Normand, C. (2006). Health workers: building and motivating the workforce. In D. T. Jamison et al. (Eds.), Disease control priorities in developing countries (2nd Ed.) (p. 7) Retrieved from https://www.ncbi.nlm.nih.gov/books/NBK11730/

Human Rights Watch. (2015). Papua New Guinea. Retrieved from https://www.hrw.org/worldreport/2016/country-chapters/papua-new-guinea

Human Rights Watch. (2016). Papua New Guinea. Retrieved from https://www.hrw.org/worldreport/2017/country-chapters/papua-new-guinea

Humphreys, S. J., Jones, P. M., Jones, A. J., \& Mara, R. P. (2002). Workforce retention in rural and remote Australia: Determining the factors that influence length of practice. Medical Journal of Australia, 176(10), 472-476. Retrieved from https://www.mja.com.au/system/files/issues/176_10_200502/hum10169_fm.pdf Huttinger, A., Dreibelbis, R., Kayigamba, F., Ngabo, F., Mfura, L., Merryweather, B., Cardon, A., \& Moe, C. (2017). Water, sanitation and hygiene infrastructure and quality in rural healthcare facilities in Rwanda. BMC Health Services Research, 17, 517. http://doi.org/10.1186/s12913-017-2460-4

Institute of National Affairs. (2007). Research of economic growth in Papua New Guinea. Retrieved from 
http://www.inapng.com/pdf_files/Research\%20of\%20Economic\%20Growth\%20in\%20P apua\%20New\%20Guinea.pdf

Jaeger, N. F., Bechir, M., Harouna, M., Moto, D. D., \& Utzinger, J. (2018). Challenges and opportunities for healthcare workers in a rural district of Chad. BMC Health Services Research, 18, 7. https://doi.org/10.1186/s12913-017-2799-6

Jayasuriya, R., Whittaker, M., Halim, G., \& Matineau, T. (2012). Rural health workers and their work environment: the role of inter-personal factors on job satisfaction of nurses in rural Papua New Guinea. BMC Health Services Research, 12, 156. http://doi.org/10.1186/1472-6963-12-156

Juan, Y-K., Cheng, Y-C., Perng, Y-H., \& Castro-Lacouture, D. (2016). International Journal of Environmental Research and Public Health, 13(7), 360. https://doi.org/10.3390/ijerph13070630

Kaur, M., Hall, S., \& Attawell, K. (2001). Medical supplies and equipment for primary health care. A practical resource for procurement and management. Retrieved from http://apps.who.int/medicinedocs/documents/s20282en/s20282en.pdf

Kiwanuka, N. S., Akulume, M., Tetui, M., Kananura, M. R., Bua, J., \& Ekirapa-Kiracho, E. (2017). Balancing the cost of leaving with the cost of living: Drivers of long-term retention of health workers: an explorative study in three rural districts in Eastern Uganda. Global Health Action, 10(4). https://doi.org/10.1080/16549716.2017.1345494

Kolehmainen-Aitken, R. (1992). The impact of decentralization on health workforce development in Papua New Guinea. Public Administration \& Development, 12, 175-191. https://doi.org/10.1002/pad.4230120205

Kora, A. (2016, October 24). Department criticised for its aging workforce. Loop PNG. Retrieved from http://www.looppng.com/content/department-criticised-its-aging-workforce Kotzee, T. J., \& Couper, I. D. (2006). What interventions do South African qualified doctors think will retain them in rural hospitals of the Limpopo province of South Africa? Rural and Remote Health Research, Education and Policy, 6(3), 681. Retrieved from https://www.ncbi.nlm.nih.gov/pubmed/16965219 
Kruk, M. E., Johnson, J. C., Gyakobo, M., Agyei-Baffour, P., Asabir, K., Kotha, S. R., Dzodzomenyo, M. (2010). Rural practice preferences among medical students in Ghana: A discrete choice experiment. Bulletin of the World Health Organization, 88, 333-341. 10.2471/BLT.09.072892

Kwansah, J., Dzodzomenyo, M., Mutumba, M., Asabir, K., Koomson, E., \& Snow, R. C. (2012). Policy talk: incentives for rural service among nurses in Ghana. Journal on Health Policy and Systems Research, 27(8), 669-676. https://doi.org/10.1093/heapol/czs016

Lagarde, M., \& Blaauw, D. (2009). A review of the application and contribution of discrete choice experiments to inform human resources policy interventions. Human Resources for Health, 7, 62. https://doi.org/10.1186/1478-4491-7-62

Landers, G. (2000). The theory - practice gap in nursing: The role of the nursing teacher. Journal of Advanced Nursing, 32(6) 1550-1556. https://doi.org/10.1046/j.13652648.2000.01605.x

Lehmann, U., Dieleman, M., \& Martineau, T. (2008). Staffing remote rural areas in middle-and low-income countries: A literature review of attraction and retention. BMC Health Services Research, 8, 19. https://doi.org/10.1186/1472-6963-8-19

Levy, C. (2005). Language research in Papua New Guinea: A case study of A war. Contemporary PNG Studies: DWU Research Journal, 2. Retrieved from http://dwu.ac.pg/en/images/Research_Journal/2005/7\%20Levy\%20Language\%20Resea rch\%20in\%20PNG\%2079-92.pdf

Loevinsohn, B. P., Guerrero, E. T., \& Gregori, S. P., (1995). Improving primary health care through systematic supervision: A controlled field trial. Health Policy Plan, 10(2), 144153. Retrieved from https://www.ncbi.nlm.nih.gov/pubmed/10143452

Macfarlane Burnet Institute. (2009, Autumn). Health issues in Papua New Guinea. Retrieved from https://www.burnet.edu.au/system/impact_newsletter/file/9/Impact__Autumn_2009.pdf

Mack, N., Woodsong, C., MacQueen, M. K., Guest, G., \& Namey, E. (2011). Qualitative research methods: A data collector's field guide. Retrieved from 
https://www.fhi360.org/sites/default/files/media/documents/Qualitative\%20Research \%20Methods\%20-\%20A\%20Data\%20Collector\%27s\%20Field\%20Guide.pdf

MacLeod, L. P. M., Stewart, J. N., Kulig, C. J., Anguish, P., Andrews, E. M., Banner, D. . . Zimmer, L. (2017). Nurses who work in rural and remote communities in Canada: a national survey. BMC Human Resources for Health, 15, 34. https://doi.org/10.1186/s12960-0170209-0

Mbemba, I. C. G., Gagnon, M-P., \& Hamelin-Brabant, L. (2016). Factors influencing recruitment and retention of healthcare workers in rural and remote areas in developed and developing countries: an overview. Journal of Public Health in Africa, 7(2), 565. https://doi.org/10.4081/jphia.2016.565

McCullough, K. M., Williams, A. M., \& Lenthall S. (2012). Voices from the bush: remote area nurses prioritise hazards that contribute to violence in their workplace. Rural and Remote Health, 12, 1972. Retrieved from https://pdfs.semanticscholar.org/c7c3/67836006a06b3200197baaa80423c438e904.pdf Ministry of Health and Social Welfare Government of Lesotho. (2010). Retention Strategy for health workforce. Retrieved from https://www.k4health.org/sites/default/files/Lesotho_Retention_Strategy.pdf

Morse, M. J., \& Field P, A. (1996). Nursing Research (pp. 1-17). Retrieved from https://doi.org/10.1007/978-1-4899-4471-9_1

Moyimane, B. M., Matlala, F. S., \& Kekana, P. M. (2017). Experiences of nurses on the critical shortages of medical equipment at a rural districts hospital in South Africa. A qualitative study. Pan African Medical Journal. 28, 100. doi:10.11604/pamj.2017.28.100.11641

Msuya, M., Blood-Siegfried, J., Chugulu, J., Kidayi, P., Sumaye, J., Machange, R. . . Pereira, K. (2017). Descriptive study of nursing scope of practice in rural medically underserved areas of Africa, South of the Sahara. International Journal of African Nursing Science, 6, 74-82. https://doi.org/10.1016/j.ijans.2017.04.003

Mullei, K., Mudhune, S., Wafula, J., Masamo, E., English, M., Goodman, C., Lagarde, M., \& Blaauw, D. (2010). Attracting and retaining health workers in rural areas: Investigating 
nurses' views on rural posts and policy interventions. BMC Health Services Research, 10, 1. $10.1186 / 1472-6963-10-S 1-S 1$

Mutale, W., Ayles, H., Bond, V., Mwanamwenge, T. M., Balabanova, D. (2013). Measuring health workerss motivation in rural health facilities: Baseline results from three study districts in Zambia. BMC Human Resources for Health, 11, 8. https://doi.org/10.1186/1478-4491-11-8

National Department of Health (2012). Health service delivery profile, Papua New Guinea. Retrieved from http://www.wpro.who.int/health_services/service_delivery_profile_papua_new_guinea National Department of Health. (2013a). Health sector human resources policy. Retrieved from http://www.health.gov.pg/publications/HRH\%20fP_IG_2014.pdf

National Department of Health. (2013b). The community health post policy. Retrieved from http://www.rphsdp.org.pg/resources/CHP_Policy.pdf

National EMTV News. (2016). PNG Health Sector Suffering. Retrieved from "http://www.emtv.com.pg/news/2016/11/png-health-sector-suffering-says-laeemergency-doctor/"rgency-doctor/

National Health and Medical Research Council. (2002). When it's right in front of you: Assisting health care workers to manage the effects of violence in rural and remote Australia. Retrieved from https://www.nhmrc.gov.au/_files_nhmrc/publications/attachments/hp16_when_its_rig ht_for_you.pdf

Nebeker, C., Simon, G., Kalichman, M., Talavera, A., Booen, E., \& Lopez-Arenas, A. (2015). Building Research Integrity and Capacity (BRIC): An Interactive Guide for Promotores/Community Health Workers. Retrieved from https://ori.hhs.gov/content/basic-research-concepts-brc

Nem, K. N. (2015, June 05). The socioeconomic effects of corruption on Papua New Guinea both present and future. Retrieved from http://www.pngblogs.com/2015/06/thesocioeconomic-effects-of-corruption.html 
Newhouse, P. R., Morlock, L., Pronovost, P., Colantuoni, E., \& Johantgen, M. (2009). Rural Hospital Nursing. Better Environments = shared vision and quality/safety engagement. The Journal of Nursing Administration, 39(4), 189-195. http://doi.org HYPERLINK "http://doi.org/10.1097/NNA.0b013e31819c9cd5"

Nursing Matters. (2015). Nurses and aging workforce. Retrieved from https://www.twna.org.tw/frontend/un07_international/file/2015/20151007.pdf Ojakaa, D., Olango, S., \& Jarvis, J. (2014). Factors affecting motivation and retention of primary health care workers in three disparate regions in Kenya. BMC Human Resources for Health, 12, 33. http://doi.org/10.1186/1478-4491-12-33

Okello, R. O. D., \& Gilson, L. (2015). Exploring the influence of trust relationships on motivation in the health sector: A systematic review. Human resources for health, 13, 16. https://doi.org/10.1186/s12960-015-0007-5

Onyx, J., \& Small, J. (2001). Memory-Work: The method. Qualitative Inquiry, 7(6), 773-786. https://doi.org/10.1177/107780040100700608. In Braun, V., \& Clarke, V. (2012) Thematic analysis. In H. Cooper, P. M. Camic, D. L. Long, A. T. Panter, D. Rindskopf, \& K. J. Sher (Eds.), APA handbook of research methods in psychology, Vol. 2: Research designs: Quantitative, qualitative, neuropsychological, and biological (pp.57-71). Washington, DC: American Psychological Association.

Oxford Business Group. (2013). Health sector struggles as Papua New Guinea's population grows. Retrieved from https://www.oxfordbusinessgroup.com/overview/obstaclesahead-health-services-sector-struggles-meet-needs-growing-population

Oxford Business Group (2018). PNG uses PPPs to improve health care delivery. Retrieved from https://oxfordbusinessgroup.com/overview/png-uses-ppps-improve-health-caredelivery

Panagariya, A. (2014). The challenges and innovative solutions to rural health dilemma. Annals of Neurosciences, 21. Retrieved from https://www.ncbi.nlm.nih.gov/pmc/articles/PMC4248476/ 
Polaschek, R. N. (1998). Cultural safety: A new concept in nursing people of different ethnicities. Journal of Advance Nursing, 27(3), 452-457. https://doi.org/10.1046/j.13652648.1998.00547.x

Provincial Infectious Diseases Advisory Committee. (2018). Best practice for environmental cleaning for prevention and control of infections (2nd Ed.). Retrieved from https://www.publichealthontario.ca/en/eRepository/Best_Practices_Environmental_Cle aning_2012.pdf

Radio New Zealand. (2018). UNICEF keen to assist rural health crisis in PNG. Retrieved https://www.radionz.co.nz/international/pacific-news/347821/unicef-keen-to-assistrural-health-crisis-in-png

Razee, H., Whittaker, M., Jayasuriya, R., Yap, L., \& Bretnall, L. (2012). Listening to the rural health workers in Papua New Guinea - The social factors that influence their motivation to work. Social Science and Medicine, 75(5), 828-835. https://doi.org/10.1016/j.socscimed.2012.04.013

Reaching for the frontier in Papua New Guinea. (2011). Retrieved from http://globetrooper.com/notes/reaching-for-the-frontier-in-papua-new-guinea/

Rourke, J. (2010). WHO Recommendations to improve retention of rural and remote health workers - important for all countries. Rural and Remote Health, 10, 1654. Retrieved from https://www.rrh.org.au/journal/download/pdf/1654

Rural Health Information hub. (2018). Education and training of the rural healthcare workforce. Retrieved from https://www.ruralhealthinfo.org/topics/workforce-education-andtraining

Rural Health West. (2013). Critical success factors for recruiting and retaining health professionals to primary health care in rural and remote locations: Contemporary review of the literature. Retrieved from http://www.ruralhealthwest.com.au/docs/defaultsource/marketing/publications/rhw_uwa_cucrh-contemporary-review-of-the-literaturef-Ir.pdf?sfvrsn=2 
Schoo, A., Lawn, S., \& Carson, D. (2016). Towards equity and sustainability of rural and remote health services access: Supporting social capital and integrated organisational and professional development. BMC Health Services Research, 16, 111. http://doi.org/10.1186/s12913-016-1359-9

Serneels, P., Montalvo, G. J., Pettersson, G., Lievens, T., Butera, D. J., \& Kidanu, A. (2010). Who wants to work in a rural health post? The role of intrinsic motivation, rural background and faith-based institutions in Ethiopia and Rwanda. Bulletin of the World Health Organization, 88. doi: 10.2471/BLT.09.072728

Sheikhi, R. M., Fallahi-Khoshnab, M., Mohammadi, F., \& Oskouie, F. (2016). Skills required for nursing career advancement: A qualitative study. Nursing and Midwifery Studies, 5(2), e30777. Retrieved from https://www.ncbi.nlm.nih.gov/pmc/articles/PMC4993070/

Simbiken, J. (2012, May 12). Nepotism will get worse if unchecked. The National. Retrieved from https://www.thenational.com.pg/nepotism-will-get-worse-if-unchecked/

Skillman, M. S., Palazzo, L., Keepnews, D., \& Hart, G. L. (2005). Characteristics of Registered Nurses in Rural vs. Urban Areas: Implications for Strategies to Alleviate Nursing Shortages in the United States (Working Paper \#91). Seattle: Center for Health Workforce Studies at the University of Washington Department of Family Medicine. Retrieved from https://pdfs.semanticscholar.org/6fec/f810cafa28a35a7624589461671d8696a6e6.pdf

Smith, P. (2013). Whose culture is it anyway? Social working within a rural community. Aotearoa New Zealand Social Work, 25 (1), 14-23. http://dx.doi.org/10.11157/anzswjvol25iss1id90

Songstad, G. N., Moland, M. K., Massay, A. D., \& Blystad, A. (2012). Why do health workers in rural Tanzania prefer public sector employment? BMC Health Services Research, 12, 92. http://doi.org/10.1186/1472-6963-12-92

Sousa, D. V., Driessnack, M., \& Mendes, A. C. I. (2007). An overview of research designs relevant to nursing: Part 1: quantitative research designs. Revista Latino-Americana de Enfermagem, 15(3), 502-507. https://dx.doi.org/10.1590/S0104-11692007000300022 
Steenbergen, K., \& Mackenzie, L. (2004). Professional support in rural New South Wales:

Perceptions of new graduate occupational therapists. Australian Journal of Rural Health, 12(4), 160-165. http://dx.doi.org/10.1111/j.1440-1854.2004.00590.x

Strasser, R. (2001). Training for rural practice. Lessons from Australia. Canadian Family Physician, 47, 2196-2198. Retrieved from https://www.ncbi.nlm.nih.gov/pmc/articles/PMC2018457/pdf/11768913.pdf

Strasser, R., \& Neusy, A-J. (2010). Context counts: Training health workers in and for rural and remote areas. Bulletin of the World Health Organization, 88(10), 777-782. http://doi.org/10.2471/BLT.09.072462

Swanson, D. (2013). PNG grapples with ageing health workforce. Irin. Retrieved from http://www.irinnews.org/report/97643/png-grapples-ageing-health-workforce

Terry, D., Le, Q., Nguyen, U., \& Haong, H. (2018). Workplace health and safety issues among community nurses: A study regarding the impact on providing care to rural consumers. BMJ, 5(8). http://dx.doi.org/10.1136/bmjopen-2015-008306

The Health Foundation. (2012). Quality improvement training for health care professionals. Retrieved from https://www.health.org.uk/sites/health/files/QualitylmprovementTrainingForHealthcar eProfessionals.pdf

The National. (2015, January 08). PNG has worst health status. Retrieved from http://www.thenational.com.pg/png-has-worst-health-status/

The World Bank. (2013). Papua New Guinea health workforce crisis: A call to action. Retrieved from http://www.worldbank.org/en/news/feature/2013/04/26/papua-new-guineahealth-workforce-crisis-a-call-to-action

The World Bank. (2017). The World Bank in Papua New Guinea. Retrieved from http://www.worldbank.org/en/country/png/overview

Thieme, K., \& Weber, M. (2014). Staff houses in rural areas - a social investment approach for hospitals. Retrieved from http://ghf.g2hp.net/2013/09/13/staff-houses-in-rural-areas-asocial-investment-approach-for-hospitals/ 
Thomason, J., \& Kase, P. (2009). Policy making in health. Studies from Papua New Guinea. In R. J. May (Ed.), Policy making and implementation (pp. 117-130). Australia National University, ANU: Sydney. Retrieved from http://pressfiles.anu.edu.au/downloads/press/p78541/pdf/ch0761.pdf

Thu, H. T. N., Wilson, A., \& McDonald, F. (2015). Motivation or demotivation of health workers providing maternal health services in rural areas in Vietnam: Findings from a mixedmethods study. Human Resources for Health, 13(1). https://doi.org/10.1186/s12960015-0092-5

Tlozek, E. (2016, December 29). Papua New Guinea villagers facing food shortages amid rising population. $A B C$. Retrieved from http://www.abc.net.au/news/2016-12-29/png-villagefacing-food-crisis-amid-rising-population/7958264

Ulutasdemir, N., \& Tanir, F. (2017). Occupational risks of health professionals. In Occupational Health (pp. 47-57). Retrieved from https://www.intechopen.com/books/occupationalhealth/occupational-risks-of-health-professionals

United Nations Development Programme. (2017). About Papua New Guinea. Retrieved from http://www.pg.undp.org/content/papua_new_guinea/en/home/countryinfo.html

United Nations Educational Scientific and Cultural Organisation world report. (2009). Investing in cultural diversity and intercultural dialogue. Retrieved from http://www.un.org/en/events/culturaldiversityday/pdf/Investing_in_cultural_diversity. pdf

Watson, A. H. A., Sabumei, G., Mola, G., \& ledema, R. (2015). Maternal health phone line: Saving women in Papua New Guinea. Journal of Personalized Medicine, 5(2), 120-139. http://doi.org/10.3390/jpm5020120

Wickberg, S. (2013, March 01). Papua New Guinea: overview of corruption and anti-corruption. Retrieved from https://www.u4.no/publications/papua-new-guinea-overview-ofcorruption-and-anti-corruption/pdf

Willis-Shattuck, M., Bidwell, P., Thomas, S., Wyness, L., Blaauw, D., \& Ditlopo, P. (2008). Motivation and retention of health workers in developing countries: A systematic review. BMC Health Services Research, 8, 247. http://doi.org/10.1186/1472-6963-8-247 
Wilson, C. (2015, February 24). Tackling corruption at its root in Papua New Guinea. Inter Press Service. Retrieved from http://www.ipsnews.net/2015/02/tackling-corruption-at-itsroot-in-papua-new-guinea/

Witter, S., Mashange, W., Namakula, J., Wurie, H. R., Ssengooba, F., \& Alonso-Garbayo, A. (2017, June). Incentives for health workers to stay in post and in rural areas: Findings from four conflict- and crisis-affected countries. Retrieved from https://rebuildconsortium.com/media/1523/hw-incentives-synthesis-wp26-120617.pdf

Women with disabilities. (2013, May 08). Women arise (Papua New Guinea) lead nationwide protest to end violence against women in PNG [Online forum]. Retrieved from https://www.facebook.com/WWDA.Australia/posts/616817648346931

Woods, M. (1997). Maintaining a nursing ethic. A grounded theory of the moral practice on experienced nurses. Massey University. NZ

World Health Organization, (2009). Increasing access to health workers in remote and rural areas through improved retention. Retrieved from http://www.who.int/hrh/migration/background_paper.pdf

World Health Organization, (2010a). Increasing access to health workers in remote and rural areas through improve retention: Global policy Recommendations. Retrieved from http://apps.who.int/iris/bitstream/handle/10665/44369/9789241564014_eng.pdf;jsessi onid=2DB2FF790FF65F7CCC067BAE16B51B34 ?sequence $=1$

World Health Organization, (2010b). Increasing access to health workers in remote and rural areas through improved retention: Global policy recommendations. Retrieved from https://www.ncbi.nlm.nih.gov/books/NBK138623/

World Health Organization. (2013). End preventable child deaths from pneumonia and diarrhoea by 2025. Retrieved from http://apps.who.int/iris/bitstream/10665/79200/1/9789241505239_eng.pdf

World Health Organization, (2014). Investing in water and sanitation: increasing access, reducing inequalities. Retrieved from http://www.who.int/water_sanitation_health/publications/glaas_report_2014/en/ 
World Health Organization, (2016). Country Corporation Strategy 2016-2020. Retrieved from http://www.who.int/countryfocus/cooperation_strategy/ccsbrief_png_en.pdf

World Health Organization, (2018a). Health and sustainable development: Energy access and resilience. Retrieved from http://www.who.int/sustainable-development/healthsector/health-risks/energy-access/en/

World Health Organization, (2018b). Health resources Availability Monitoring System (HeRAMS). Retrieved from http://www.who.int/hac/herams/en/

World Health Organization, (2018c). Occupation health. Retrieved from http://www.who.int/occupational_health/topics/hcworkers/en/

World Vision. (2013). Papua New Guinea health and human well-being. Retrieved from "https://www.worldvision.com.au/docs/default-source/school-resources/globaleducation-papua-new-guinea.pdf?sfvrsn=2"dvision.com.au/docs/default-source/schoolresources/global-education-papua-new-guinea.pdf?sfvrsn=2

Wurie, H. R., Samai, M., \& Witter, S. (2016). Retention of health workers in rural Sierra Leone: Findings from life histories. Human Resources for Health, 14, 3. http://doi.org/10.1186/s12960-016-0099-6

Zhan, X., Zhang, Z., Sun, F., Liu, Q., Peng, W., Zhang, H., \& Yan, W. (2017). Effects of improving primary health care workers' knowledge about public health services in rural China: A comparative study of blended learning and pure e-Learning. Journal of Medical Internet Research, 19(5). Retrieved from http://www.jmir.org/2017/5/e116/ 